Clinical presentation of patients who developed narcolepsy type 1 after the influenza A (H1N1) epidemic and vaccination campaign in Norway

-Health related quality of life, psychiatric symptoms and medical comorbidities

Sebjørg Elizabeth Hesla Nordstrand

The degree philosophiae doctor (ph.d)

2020

Faculty of Medicine

Oslo University Hospital

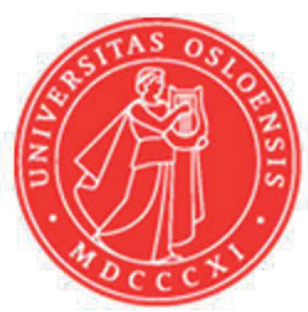

Norwegian Centre of Expertise for

Neurodevelopmental Disorders

and Hypersomnias

Oslo University Hospital 
(C) Sebjørg Elizabeth Hesla Nordstrand, 2020

Series of dissertations submitted to the Faculty of Medicine, University of Oslo

ISBN 978-82-8377-715-4

All rights reserved. No part of this publication may be reproduced or transmitted, in any form or by any means, without permission.

Cover: Hanne Baadsgaard Utigard.

Print production: Reprosentralen, University of Oslo. 
"Innocent sleep. Sleep that soothes away all our worries. Sleep that puts each day to rest. Sleep that relieves the weary laborer and heals hurt minds. Sleep, the main course in life's feast, and the most nourishing."

William Shakespeare, Macbeth 


\section{TABLE OF CONTENTS}

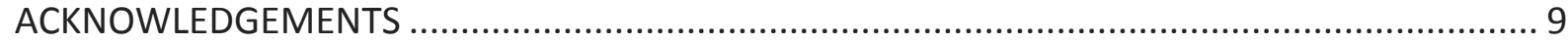

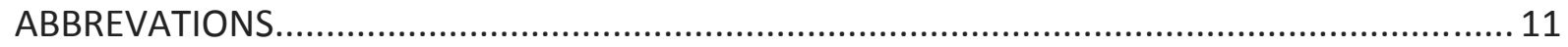

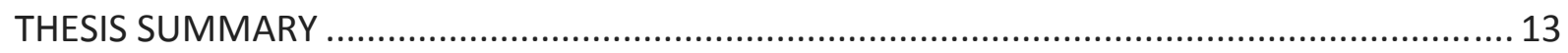

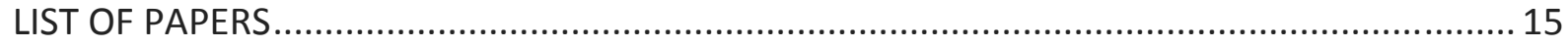

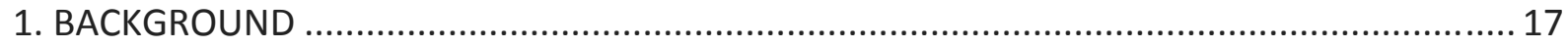

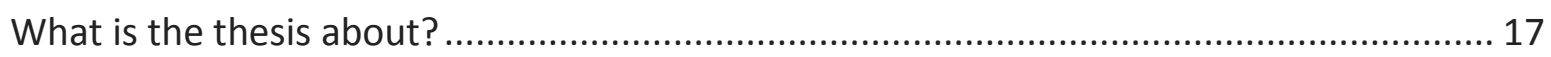

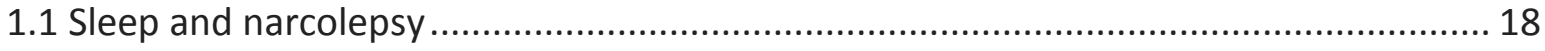

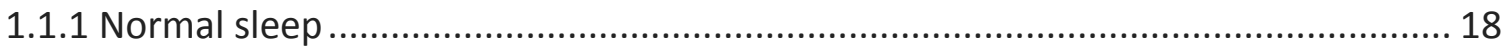

1.1.2 Narcolepsy - from a psychiatric condition to a somatic disorder. A short historical

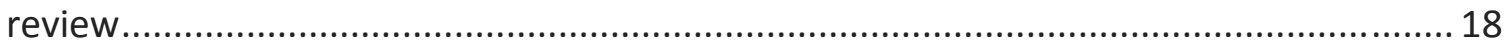

1.1.3 Classification and diagnostic criteria for narcolepsy .......................................... 19

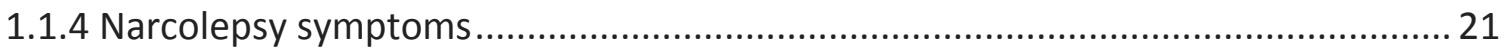

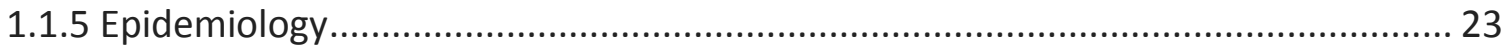

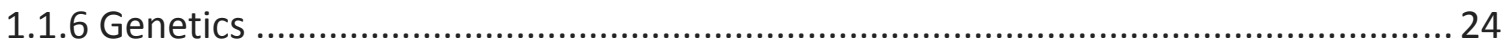

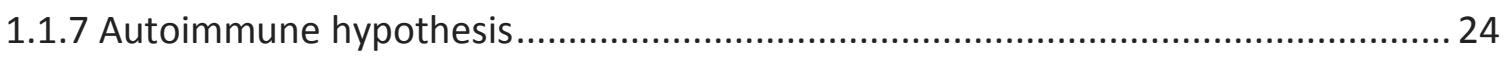

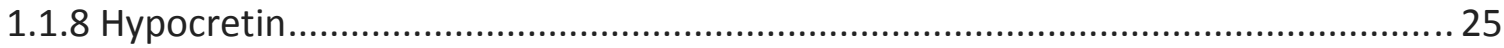

1.2 Narcolepsy after the influenza A (H1N1) epidemic and vaccination campaign ............. 27

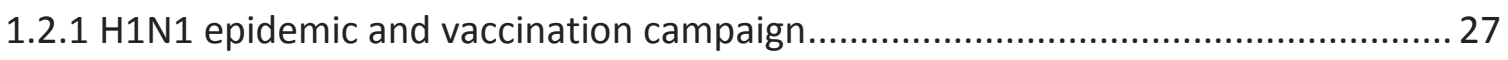

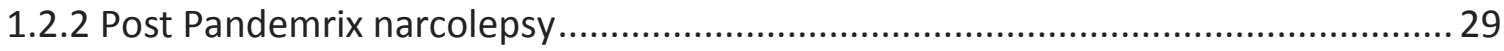

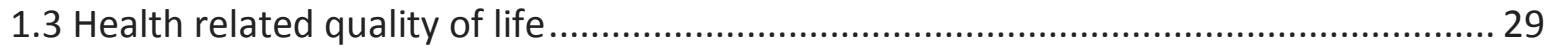

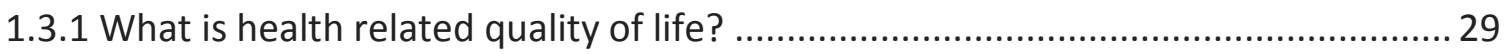

1.3.2 Health related quality of life in narcolepsy.......................................................... 30

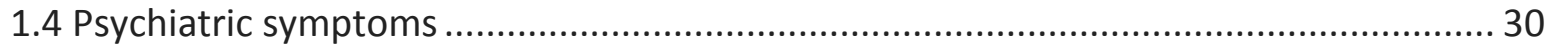

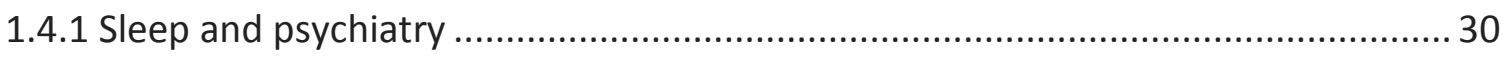

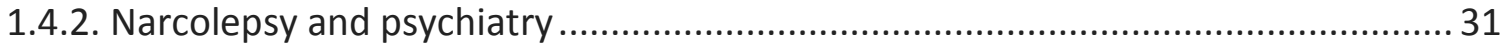

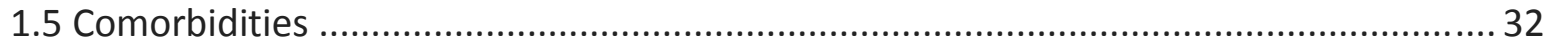

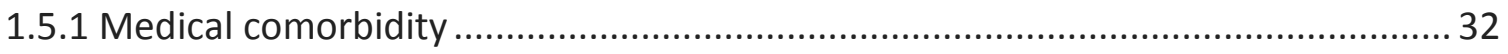

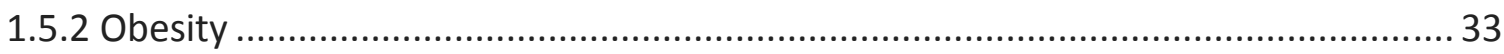

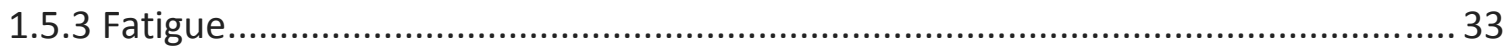

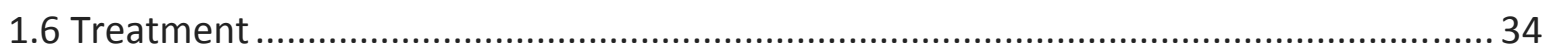

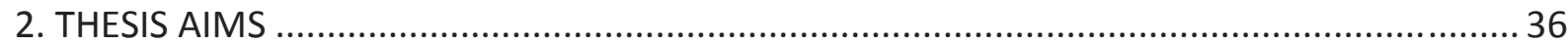




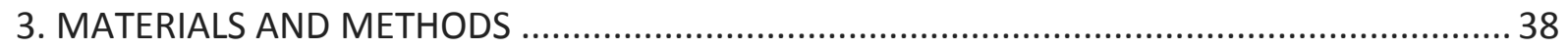

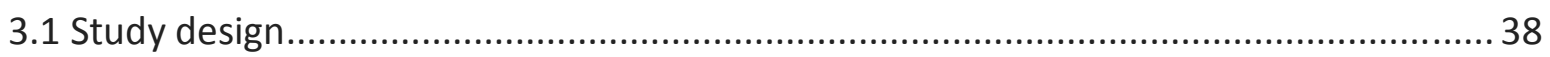

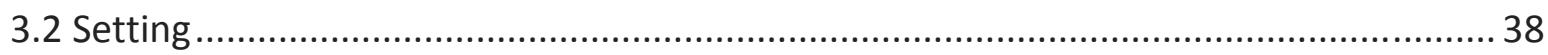

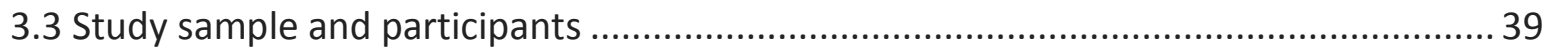

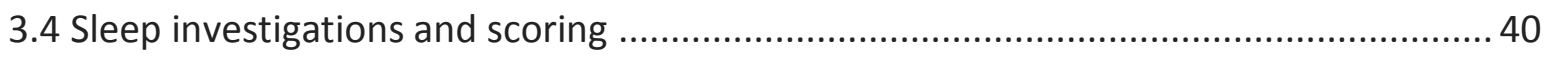

3.5 Anthropometric measurements and blood pressure............................................. 42

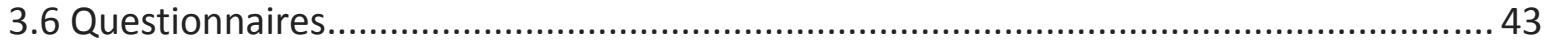

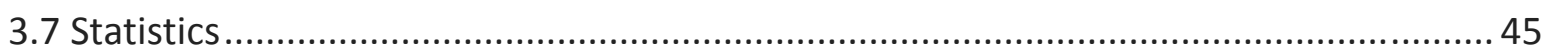

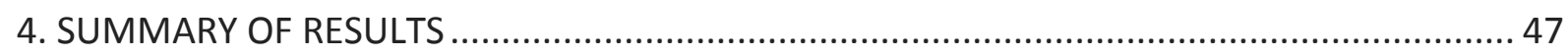

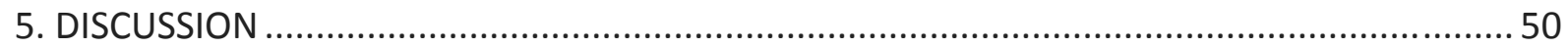

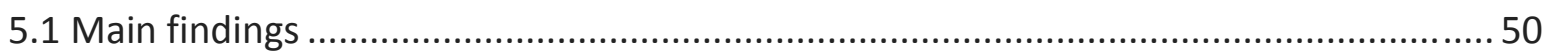

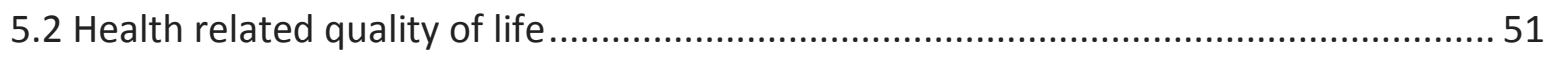

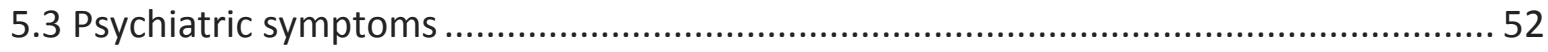

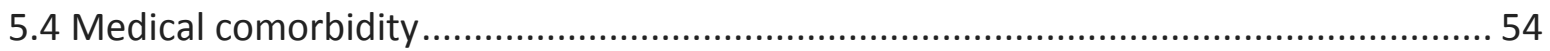

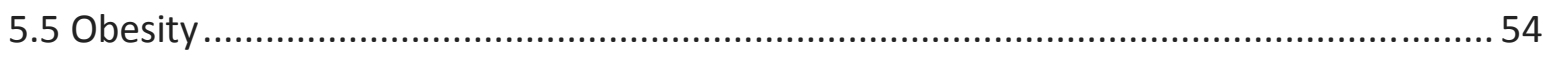

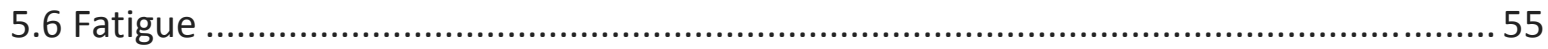

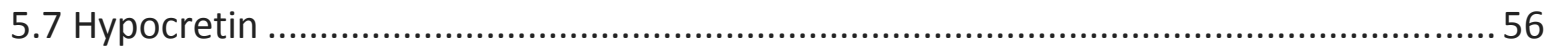

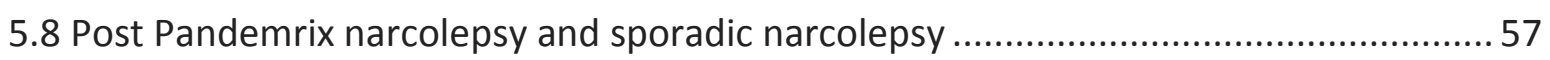

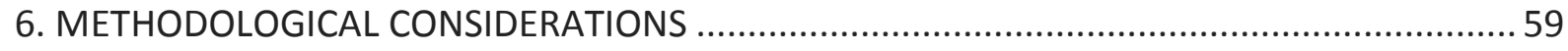

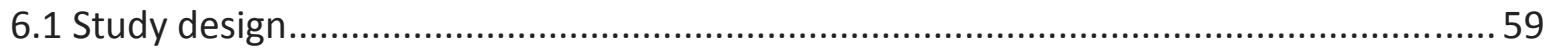

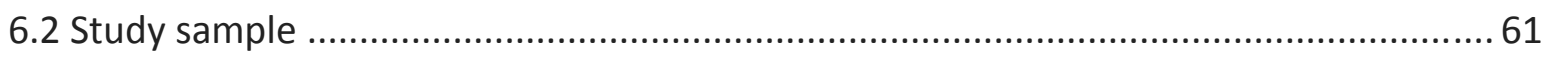

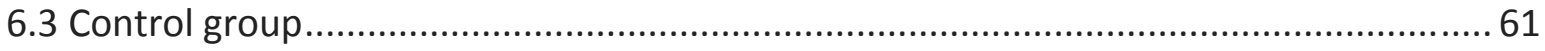

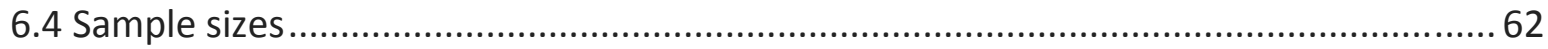

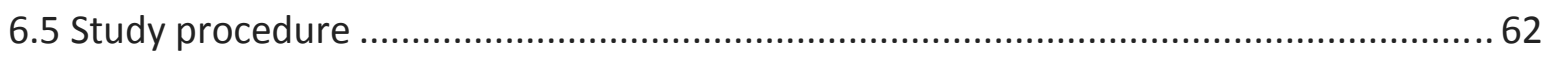

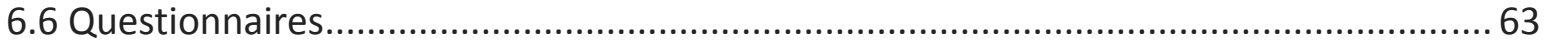

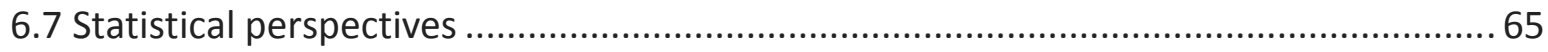

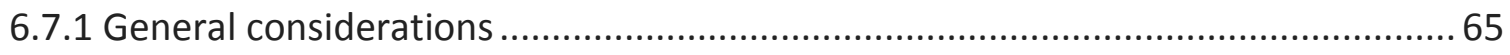

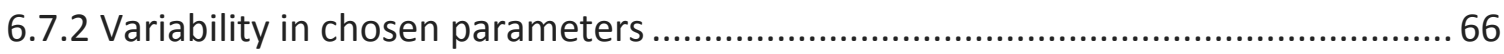

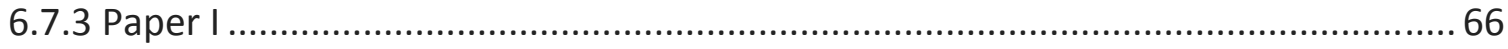

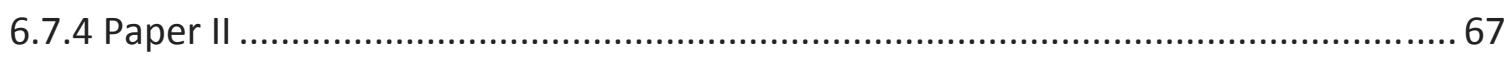

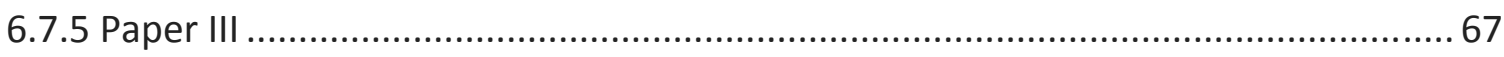

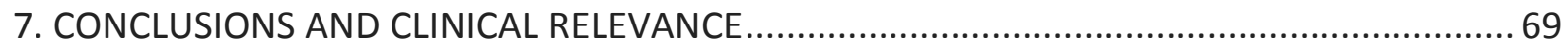




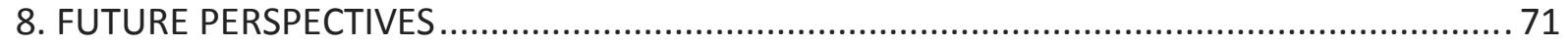

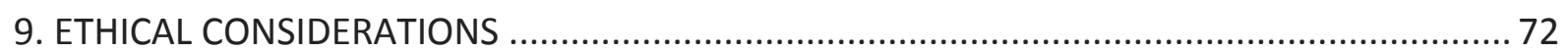

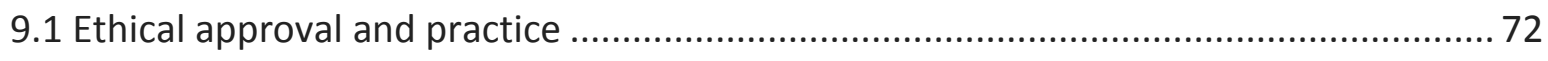

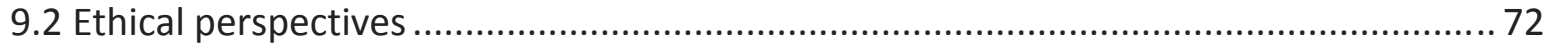

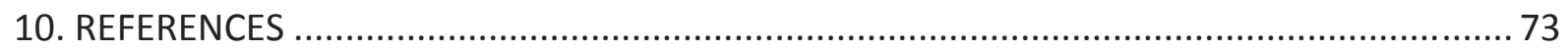

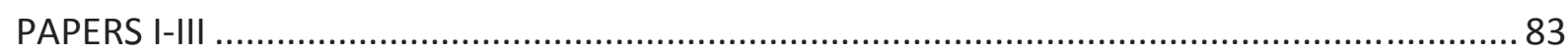




\section{ACKNOWLEDGEMENTS}

Writing this thesis has been fascinating and extremely rewarding. I would like to thank a number of people who have contributed to the final result in many different ways. To commence with, I would like to thank the patients and their relatives for participating in the studies. I would like to thank the Norwegian Centre of Expertise for Neurodevelopmental Disorders and Hypersomnias, (NevSom), Department of Rare Disorders, Division of Paediatric and Adolescent Medicine, Oslo University Hospital (OUH) and the Institute of Clinical Medicine, University of Oslo for the opportunity to complete this project. Research support was provided by the Norwegian Ministry of Health and Care Services, and studies included in the thesis were supported by the Research Council of Norway and South-Eastern Norway Regional Health Authority.

I would like to express my deep gratitude to my main supervisor, Dr. Stine Knudsen Heier, at NevSom, for all the work and effort she has invested in this project, for the enthusiastic encouragement and useful critiques. Without her expertise, it would have not been possible to complete this project. I am very thankful to my co-supervisor, Dr. Berit Hjelde Hansen at NevSom, for her valuable suggestions, her constant encouragement, understanding and invaluable support. I owe an enormous gratitude to my co-supervisor, Professor Terje Rootwelt, Head of Division of Paediatric and Adolescent Medicine, OUH, for his outstanding knowledge and expertise, for his healthy and constructive advice, for thoroughly reading through all the drafts and for always finding time to answer even the smallest questions. I am deeply grateful to my co-supervisor, Dr. Kristian Bernhard Nilsen, Head of Section of Clinical Neurophysiology, OUH, for his clear thinking and valuable comments, for all the help, particularly in regard to sleep investigations. I would also like to thank my co-supervisor, TorIvar Karlsen at the Faculty of Health and Sports Sciences, University of Agder, for constructive comments and suggestions, particular in issues concerning health related quality of life. I would like to offer my special thanks to David Swanson at Oslo Centre for Biostatistics and Epidemiology, OUH, for his engagement in this project, for thoroughly looking into the statistics and the drafts of the papers. Yusman Kamaleri helped with the statistics and the planning of the project, for which I am grateful. Further, I would like to thank our collaborates at the Hormone Laboratory, OUH, in particular Ranveig $\varnothing$ strem and co-author Dr. Per M. Thorsby for their contribution to collecting and analyzing data. I would 
like to thank my friends and coworkers at NevSom for their encouragement, especially Hilde Therese Juvodden, Rannveig Viste and Janita Vevelstad, whom all have contributed greatly to this project. My former collogues, Hilde Andresen and Knut Bronder were a part of the beginning of the project, and the time they invested in this project has been deeply appreciated.

I am deeply grateful to my friends and family for their endless support. All the treasured moments together have helped me through the ups and downs. I would like to take this opportunity to express my affectionate gratitude toward my parents. Thanks to my father, Per Egil, for his invaluable support and for teaching me never to give up, and to my late mother, Molly, whose words of wisdom I recall every day. From my whole heart, I thank our four boys, Ola Marcellus, Johannes Teofilo, Jona Louis and Asle Ezzard for each day reminding us what is important in life. Finally, I have written many words these past years, but words describing my gratitude towards my husband, Njord, are hard to find. He has been endlessly patient, and his fighting spirit has inspired me and helped me through this PhD project. I thank him for his never-ending support and for being life's best mentor. 


\section{ABBREVATIONS}

\begin{tabular}{|c|c|}
\hline AASM & American Academy of Sleep Medicine \\
\hline ADHD & Attention deficit hyperactivity disorders \\
\hline ASEBA & Achenbach System of Empirically Based Assessment \\
\hline ASR & Adult Self Report \\
\hline BMI & Body mass index \\
\hline CBCL & Child Behavior Checklist \\
\hline CSF & Cerebrospinal fluid \\
\hline EDS & Excessive daytime sleepiness \\
\hline EEG & Electroencephalography \\
\hline EES & Epworth Sleepiness Scale \\
\hline EMG & Electromyography \\
\hline $\mathrm{HH}$ & Hypnagogic hallucinations \\
\hline HLA & Human leucocyte antigen \\
\hline HRQoL & Health related quality of life \\
\hline ICSD & International Classification of Sleep Disorder \\
\hline $\mathrm{IH}$ & Idiopathic hypersomnia \\
\hline IOTF & International Obesity Task Force \\
\hline $\mathrm{MCH}$ & Major histocompatibility complex \\
\hline MSLT & Multiple sleep latency test \\
\hline
\end{tabular}

NevSom Norwegian Center of Expertise for Neurodevelopmental Disorders and Hypersomnias 
NT1 Narcolepsy type 1

NT2 Narcolepsy type 2

OSAS Obstructive sleep apnea syndrome

OUH Oslo University Hospital

PedsQL Pediatric Quality of Life ${ }^{\mathrm{TM}}$ Generic Core Scales 4.0

PPN Post Pandemrix narcolepsy

PSG Polysomnography

REM Rapid eye movement

RLS Restless legs syndrome

SN Sporadic narcolepsy

SOREM Sleep onset rapid eye movement

SP Sleep paralysis

SSQ Stanford Sleep Questionnaire

SWS Slow wave sleep

WHO World Health Organization 


\section{THESIS SUMMARY}

Narcolepsy type 1 (NT1) is a chronic sleep disorder characterized by excessive daytime sleepiness, (EDS), hypnagogic/hypnopompic hallucinations $(\mathrm{HH}$, hallucinations either when falling asleep or when waking up), sleep paralysis (SP, paralysis when falling asleep and/or when waking up), cataplexy (sudden loss of muscle tone triggered by strong emotions) and fragmented nocturnal sleep. NT1 is believed to be caused by autoimmune destruction of hypocretin producing neurons in the lateral hypothalamus. NT1 may be complicated by psychiatric and medical comorbidities in addition to impaired health related quality of life (HRQoL).

After the H1N1 influenza epidemic and vaccination campaign with Pandemrix in 2009, several countries, including Norway, experienced an increase in the incidence of NT1. There were initially some indications that the phenotype was somewhat different and the use of the terms post Pandemrix narcolepsy (PPN) and sporadic narcolepsy (SN) was initiated. In PPN, the onset was thought to be more abrupt and symptoms more severe and more often accompanied by additional comorbidities such as psychiatric symptoms. Studies have, however, not been able to show major significant differences between PPN and SN. The overall aim of this thesis was to explore the phenotype in patients who developed NT1 after the H1N1 epidemic and vaccination campaign in Norway. We focused primarily on exploring 1) changes in HRQoL over time 2) the extent of psychiatric symptoms and 3) the extent of medical comorbidities, obesity in particular, as well as fatigue.

The patients included in this thesis had developed NT1 symptoms after H1N1 the influenza epidemic and vaccination campaign with Pandemrix in 2009. The inclusion period was from February 2014 to September 2017. All participants were hospitalized and underwent sleep investigations, physical examination and completed semi-structured clinical interviews and surveys.

NT1 is a disabling disorder and several studies report an impaired HRQoL in NT1 patients. However, studies exploring HRQoL over time are sparse. In Paper I, we show that HRQoL is impaired, but we also show that HRQoL improves after 2 years of follow up. The reason for the high prevalence of psychiatric symptoms seen in patients with SN and PPN is debated, 
and explanations may be multifactorial. In Paper II, we confirm a high prevalence of psychiatric symptoms which in our cohort was mainly driven by a high prevalence of internalizing symptoms. Medical comorbidity, particularly obesity is a well established complication to NT1. The reason for the high prevalence of obesity is, however, not fully understood. In support of previous studies, in Paper III we found a high prevalence of overweight and obesity in both children and adults.

In conclusion, the findings in this thesis show that the NT1 phenotype in patients who developed NT1 after the H1N1 epidemic and vaccination camping in Norway is complex and associated with several comorbidities and impaired HRQoL. Further, our findings are similar to other comparable studies on both PPN and SN narcolepsy populations. This thesis may improve the understanding of the narcolepsy phenotype, potentially leading to an earlier diagnosis and improved treatment. Further, it emphasizes the importance of a multidisciplinary approach to prevent secondary complications and improve general health and HRQoL. 


\section{LIST OF PAPERS}

\section{Paper I}

Nordstrand SEH, Hansen BH, Kamaleri Y, Nilsen KB, Rootwelt T, Karlsen TI, Knudsen S. Changes in quality of life in individuals with narcolepsy type 1 after the H1N1-influenza epidemic and vaccination campaign in Norway: a two-year prospective cohort study. Sleep Medicine. 2018.

Paper II

Nordstrand SEH, Hansen BH, Rootwelt T, Karlsen TI, Swanson D, Nilsen KB, Knudsen S. Psychiatric symptoms in patients with post-H1N1 narcolepsy type 1 in Norway. Sleep. 2019.

Paper III

Nordstrand SEH, Juvodden HT, Viste R, Rootwelt T, Karlsen TI, Thorsby PM, Swanson D, Nilsen KB, Hansen BH, Knudsen SH. Obesity and other medical comorbidities among NT1 patients after the Norwegian H1N1 influenza epidemic and vaccination campaign. Sleep. 2019. 


\section{BACKGROUND}

\section{What is the thesis about?}

Narcolepsy type I (NT1) is a chronic neurological sleep disorder (1) most likely caused by an autoimmune mediated destruction of hypocretin producing neurons that produce the wakesleep regulation neurotransmitter hypocretin (2). Norway, in line with several other countries experienced an increased incidence of NT1 after the H1N1 influenza epidemic and vaccination campaign in 2009 (3-5). Due to a perceived more abrupt debut of symptoms, high severity of symptoms and additional psychiatric symptom, it was speculated that narcolepsy after Pandemrix vaccination, post Pandemrix narcolepsy (PPN), was different from the previously known narcolepsy, sporadic narcolepsy (SN) $(5,6)$.

Typical NT1 symptoms are excessive daytime sleepiness (EDS), hypnagogic/hypnopompic hallucinations ( $\mathrm{HH}$, hallucinations either when falling asleep or when waking up), sleep paralysis (SP, paralysis when falling asleep and/or when waking up), cataplexy (sudden loss of muscle tone triggered by strong emotions) and fragmented nocturnal sleep (1). Clinical manifestations may vary with age, and children may present with atypical symptoms such as hyperactivity, atypical cataplexy and obesity (7). Moreover, studies have shown a high prevalence of psychiatric $(8,9)$ and medical comorbidities $(8,10)$, in particular obesity (1113), as well as fatigue (14). The variation in phenotype may lead to misdiagnosis, substantial delay in diagnosis and may have major implications for health related quality of life (HRQoL) $(15,16)$.

The primary aim of this thesis was to explore the NT1 phenotype in patients who developed narcolepsy after the H1N1 epidemic and vaccination campaign in Norway in 2009, focusing on HRQoL (Paper I), psychiatric symptoms (Paper II) and medical comorbidity (Paper III). A better general understanding of the narcoleptic phenotype may improve our clinical approach and treatment. 


\subsection{Sleep and narcolepsy}

\subsubsection{Normal sleep}

Humans spend about one-third of their lives asleep, however, the reason why we sleep is highly debated and not fully understood $(17,18)$. Disturbances in sleep pattern, and absence of sleep have major physiological consequences and is associated with psychiatric and medical disorders $(19,20)$. Sleep pattern and circadian rhythm changes over a life span, and the main controlling center regulating the circadian rhythm consists of brain cells in the suprachiasmatic nucleus (21). The regulation of sleep-wakefulness is normally not fully matured in infants, and development of the sleep-wake stability involves a shift from the disturbed and fragmented sleep across the day in infants to consolidated sleep at night in early childhood $(17,21)$.

There are two types of sleep, rapid eye movement (REM) sleep and non- REM (NREM) sleep. NREM is further dived into sleep stage 1, 2 and 3 which reflect the depths of our sleep. Sleep architecture refers to the cyclic alternation between REM sleep and NREM sleep throughout the night, each cycle lasting 60-90 minutes. A normal sleep pattern will typically begin with NREM sleep stage 1, lasting from 1-7 minutes. Sleep stage 1 accounts for 2-4\% of a total sleep period. Sleep stage 2 lasts from 1-20 minutes and accounts for $45-55 \%$ of a total sleep period. In sleep stage 3, also called slow wave sleep (SWS), the sleep is deep so that more stimuli is needed to be awakened than in the previous stages. Most of the SWS occurs in the first part of the night, lasting up to 40 minutes. $13-23 \%$ of a total sleep period is SWS. In a normal sleep pattern REM sleep usually occurs at the end of each sleep cycle and $20-25 \%$ of a total sleep period is REM sleep. Although dreams may also occur in NREM sleep, most of our dreams occur in the REM sleep phase. REM sleep is associated with muscle atonia, this is to avoid "living out our dreams"(17).

\subsubsection{Narcolepsy - from a psychiatric condition to a somatic disorder. A short historical review}

The first to describe narcolepsy with cataplexy was the German professor Karl Friedrich Otto Westphal (1833-1890) in 1877 (22). Narcolepsy was at that time believed to be a disorder of psychiatric origin, and literature refers to the case of Von Zatostrow. He was a famous 
criminal rapist, and it was believed that his excessive sleepiness was a result of repressed homosexuality associated with shame (23). Some years later, in 1880, the French physician Jean Baptiste Edouard Gélineau (1828-1906) described a male patient with up to 200 sleep attacks per day. He considered the term "neurosis", as an independent disorder distinct from epilepsy, hence, named the disorder narcolepsy (24). Several years later, in 1902, Loëwenfeld gave the symptom of muscle weakness, triggered by emotions, the name "cataplexy" (25). In relation to the Spanish flu in 1917-1927, the Austrian psychiatrist and neurologist Constantin Freiherr von Economo (1876-1931) described the condition of Encephalitis Letargica (26). He described symptoms bearing resemblance to those of narcolepsy with cataplexy. Narcolepsy was at that time often used to describe any form of daytime sleepiness (23). Furthermore, he described additional psychiatric symptoms. Oculomotor paralysis was also associated with Encephalitis Letargica, which led Von Economo, as the fist in history, to suggest that the posterior hypothalamus was injured in patients with narcolepsy $(23,26)$.

\subsubsection{Classification and diagnostic criteria for narcolepsy}

The narcolepsy described by Westphal and Gélineau did not distinguish narcolepsy with cataplexy from narcolepsy without cataplexy. It was believed that there was only one single type of narcolepsy independent of the presence of cataplexy. This was the case for many years, and only when the diagnostic coding manual for sleep disorders; the International Classification of Sleep Disorders (ICSD), 2.nd edition was published in 2005, was narcolepsy officially separated into narcolepsy with and without cataplexy (27). As scientists have come closer to understanding the etiology and pathophysiology of narcolepsy even further classifications have been made. There is a very close association between narcolepsy with cataplexy and a particular human leucocyte antigen allele ((HLA)-DQB1*06:02)(28), as well as with low levels of the neuropeptide hypocretin type 1 in cerebrospinal fluid (CSF) (29). The same associations are not seen in patients without cataplexy. Hence, based on these findings, the current International Classification of Sleep Disorders 3.rd edition (ICSD-3), published in 2014, classifies narcolepsy into narcolepsy type 1 (NT1) and narcolepsy type 2 (NT2) (Table 1). Of note, not all patients with NT1 (with hypocretin deficiency) have cataplexy at the time of diagnosis, cataplexy may appear later on or may be very subtle (30, 
31). Narcolepsy may also be secondary to other disorders such as brain tumors (32), multiple sclerosis (33), head injuries (34) and encephalitis (35).

Table 1. Diagnostic criteria based on the International Classification of Sleep Disorders, American Academy of Sleep Medicine 3.rd edition

Narcolepsy type 1 (with hypocretin deficiency) - both of the following criteria must be met:

1. The patient has daily periods of irrepressible need to sleep or daytime lapses into sleep, occurring for at least 3 months.

2. The presence of one or both of the following:

1. Cataplexy and a MSL $\leq 8$ minutes and 2 or more SOREMPs on MSLT performed according to standard techniques. A SOREMP on the preceding nocturnal PSG (i.e., REM onset within 15 minutes of sleep onset) may replace one of the SOREMPs on the MSLT.

2. CSF hypocretin 1 concentration is either $\leq 110 \mathrm{pg} / \mathrm{ml}$ or $<1 / 3$ of mean values obtained in normal subjects with the same standardized assay

Narcolepsy type 2 (without hypocretin deficiency) - all 5 of the following criteria must be met:

1. The patient has daily periods of irrepressible need to sleep or daytime lapses into sleep occurring for at least 3 months.

2. A mean sleep latency $\leq 8$ minutes and 2 or more SOREMPs on MSLT performed according to standard techniques. A SOREMP (within 15 minutes of sleep onset) on the preceding nocturnal PSG may replace one of the SOREMPs on the MSLT.

3. Cataplexy is absent.

4. Either CSF hypocretin 1 concentration has not been measured or CSF hypocretin 1 concentration is $>110 \mathrm{pg} / \mathrm{ml}$ or $>1 / 3$ of mean values obtained in normal subjects with the same standardized assay.

5. The hypersomnolence and/or MSLT findings are not better explained by other causes such as insufficient sleep, obstructive sleep apnea, delayed sleep phase disorder, or the effect of medication or substances or their withdrawal. 


\subsubsection{Narcolepsy symptoms}

The typical symptoms in patients with narcolepsy are EDS, cataplexy, $\mathrm{HH}$ and SP $(1,30)$ and fragmented sleep (36). Other features such as micro-sleep (sleep-attacks lasting for only a few seconds), automatic behavior and sleep drunkenness (episodes of confusion) are recognized as additional symptoms $(30,37)$.

\section{Excessive daytime sleepiness}

EDS is the main symptom in narcolepsy and is mandatory for the diagnosis (1). The extent of sleepiness varies among patients, and the severity of the sleepiness fluctuates during the day. Typically, it is described as an impossibility to stay awake, and small involuntary sleep attacks will occur during the day. The sleep attacks seldom come abruptly and are preceded by periods of drowsiness. In contrast to children with narcolepsy, adult patients normally feel recovered for a short time after involuntary sleep attacks or after taking voluntary naps (30). Active stimulation and physical activity may be ways to cope with the tiredness.

\section{Cataplexy}

Cataplexy is characteristic for NT1. It is defined as sudden paralysis of muscles triggered by emotions, however, consciousness is preserved. Typically emotions triggering cataplexy are laughter, excitement and anger. Patients may have partial cataplectic attacks which may or may not develop into a total cataplectic attack and collapse of the body. All striated muscles may be involved, however, eye movement and respiratory muscles are not affected (36). The expression of cataplexy varies between patients and may be subtle and therefore difficult to recognize. Children may present with head-nodding and tongue protrusion as the only expression $(30,37,38)$. 


\section{$\underline{\text { Hypnagogic and hypnopompic hallucinations }}$}

Hypnagogic hallucinations and hypnopompic hallucinations are hallucinations that occur in the transitional period either when falling asleep or when waking up, respectively. The prevalence of $\mathrm{HH}$ in patients with narcolepsy is reported to be around $30 \%(36)$. This phenomenon may also occur in healthy individuals (39). The images are often unpleasant and bizarre. They are distinct from nightmares and may be terrifying for the patient. Hallucinations may be visual, auditory or tactile. The hallucinations may be misinterpreted as psychotic disorders. In contrast to $\mathrm{HH}$, psychotic hallucinations appear when being fully awake, but since patients with narcolepsy have sleep attacks, including REM sleep, during the day, differentiating between the two may be difficult $(36,39)$.

\section{$\underline{\text { Sleep paralysis }}$}

Sleep paralysis is the inability to move either right after waking up or just before falling asleep. The attacks may last for seconds to minutes. Sleep paralysis may accompany with $\mathrm{HH}$ potentially making the hallucinations even more frightening (30). Even the smallest movements like opening an eye can be difficult. The atonia is similar to the muscle atonia seen in complete cataplectic attacks (36).

\section{$\underline{\text { REM sleep phenomenon }}$}

Cataplexy, SP and $\mathrm{HH}$ are thought to be REM sleep related phenomena, implying that REM sleep may interfere with different sleep stages and wakefulness. Neurophysiological studies indicate that cataplexy shares a common mechanism with REM sleep atonia (30). Although the mechanism is not fully established, the absence of hypocretin signaling may lead to dysregulated transition between sleep stages and wakefulness as hypocretin is hypothesized to work as a stabilizer between sleep and wakefulness. However, this theory alone cannot fully explain why cataplexy is triggered by emotions. A plausible explanation is that the hypocretin neurons are involved in the regulation of the area in the brain responsible for muscle atonia (2). Healthy individuals without narcolepsy may also feel weakening in muscle groups when laughing or when experiencing a strong emotion (39). 


\section{Fragmented sleep}

Regulating sleep-wakefulness and maintaining sleep stage stability are the central downstream effects of the hypocretin-system. The lack of functional hypocretintransmission leads to severe sleep-wake fragmentation, such as seen in NT1. Fragmented sleep is associated with behavior problems and poorer school functioning in healthy children and in patients with other sleep disorders such as in obstructive sleep apnea syndrome (OSAS) and restless legs syndrome (RLS) (40-42). Moreover, psychiatric disorders such as depression and anxiety may be accompanied by sleep disturbances such as frequent awakenings (43).

\subsubsection{Epidemiology}

Most studies of the prevalence of narcolepsy contain a mixture of patients with NT1 and NT2, hence the true prevalence of NT1 is to the best of our knowledge not well documented. Nevertheless, the estimated total prevalence of narcolepsy in Western countries is $20-50$ per $100,000(44,45)$. The prevalence of narcolepsy is higher in Japan than in Europe and North America and lower in Israel (30). There are few published studies on the incidence of narcolepsy. Silber et al. reported an incidence rate of narcolepsy with cataplexy of 0.74 per 100000 per year over a 30 year period based on a US-population (46). Wijnans et al. reports a stable incidence rate of 1 per 100000 per year in a European based population (45). After the H1N1 influenza epidemic and vaccination campaign, there was an increase in the incidence rate in several counties, especially in children and adolescents aged 5-19 years (3$5,47)$. Sweden reported a 25 fold increase in incidence (4), Finland detected a 13-fold increase $(5,48)$ and Norway a 10 fold increase $(3)$ in incidence after the H1N1 epidemic and vaccination campaign. Males have been reported to have a slightly higher risk of NT1 than females (1.2 males per 1 female) (46). The debut of symptoms varies with a bimodal distribution, including a large peak around 15 years of age and a smaller peak around 36 years (49). Narcolepsy is associated with a long diagnostic delay (50), which may affect the prevalence numbers. Moreover, there seems to be a seasonal variation in narcolepsy. Dauvilliers et al. showed that subjects born in March have significantly higher narcolepsy rates, and the occurrence of onset is seasonal, typically highest in spring and summer (51). 


\subsubsection{Genetics}

There is a $2.8 \%-5.6 \%$ risk of NT1 in first degree relatives, indicating the important role of genetics in developing NT1 (52). The first discovery of a genetic association was made by a Japanese group led by Dr. Honda in 1983 who found an association between narcolepsy and the HLA gene, HLA-DR2 (53).

HLA genes encode the different antigen-presenting major histocompatibility complex (MHC) molecules which are cell surface proteins essential for the acquired immune system to recognize foreign molecules. The main genetic risk factor for narcolepsy is the HLADQB1*06:02 allele encoding MCH class II $(2,28)$. Studies have shown that $98 \%$ of NT1 patients carry the HLA-DQB1*06:02 allele. For the $2 \%$ of NT1 patients that do not carry the allele, other mechanism may be involved (28). HLA-DQB1*06:02 is carried by approximately $18 \%$ of the general Norwegian population (54).

\subsubsection{Autoimmune hypothesis}

Parkes et al. suggested in 1986 that the immune system could be involved in the pathogenesis of narcolepsy (55). An autoimmune selective destruction of hypocretin neurons localized in the posterior hypothalamus is now the leading theory as the cause of NT1 $(56,57)$. There are several indications for this hypothesis other than the strong association between NT1 and HLA-DQB *0602. Studies have shown that infections may precede the debut of narcolepsy symptoms indicating an immune mediated reaction (58). A study reported that an antibody against tribbles homologe 2 (TRIB2), a protein produced by hypocretin cells among other cells, are present in patients with NT1 (59). However, the direct causative role of TRIB2 in the development of narcolepsy is questioned (2), and this finding was not confirmed in a study of patients with PPN (60). The mechanism leading to the destruction of hypocretin cells is not known. It is suggested by Kornum et al. that the most important components include T cells (immune responsive cells), microglia (cells in brain and spinal cord) and antibodies. Antigen presentation by MHC class II molecules to $\mathrm{CD}^{+} \mathrm{T}$ cells (helper cells) is central in the pathophysiology of NT1, by triggering an immune response. The possible mechanism of the destruction of hypocretin neurons is that autoreactive $\mathrm{T}$ cells and antibodies enter the brain and that $\mathrm{T}$ cells or microglia release 
cytokines and chemokines (cell signaling proteins). This will lead to the invasion of more immune cells damaging the hypocretin neuros or making them vulnerable to attack from $\mathrm{CD}^{+} \mathrm{T}$ cells (killer cells) leading to neuronal loss. $\mathrm{CD} 4^{+} \mathrm{T}$ cells can enter a healthy brain, however, antibodies only cross the blood-brain barrier if the blood-brain barrier is impaired in one way or another, potentially due to an infection (2).

\subsubsection{Hypocretin}

Hypocretin, also called orexin, was discovered in 1998 by two independent research groups. De Lecea and coworkers (61) named the neuropeptide hypocretin as it was localized in the posterior lateral hypothalamus and resembled the gut-hormone secretin, while Sakurai and coworkers (62) named the neuropeptide orexin as it increased appetite. Even though both names are used, hypocretin is in many settings, especially in regard to narcolepsy, the preferred. Hypocretin consists of 2 peptides (hypocretin 1 (also called orexin A), and hypocretin 2 (also called orexin B)) produced from the same precursor (preprohypocretin) in approximately 70.000 neurons located in the perifornical lateral hypothalamus (63). In 1999, Lin and coworkers discovered that canine narcolepsy was caused by a mutation in the hypocretin 2 receptor (64). A year later Nishino et al. (65) reported that hypocretin levels in cerebral spinal fluid (CSF) were low in patients with narcolepsy, and Thannickal et al. reported reduced amount of hypocretin neurons in the hypothalamus in narcolepsy patients (66).

\section{$\underline{\text { Hypocretin and arousal }}$}

The hypocretin-system plays a role in the regulation of sleep-wake transition, but the various effects on these processes are regulated by different brain regions and systems. Research indicates that hypocretin acts as a coordinator for maintenance of sleep and wakefulness and the transition between these, rather than generating sleep directly (67). The pathways through which it interacts are thought to be via many neurotransmitter systems $(29,67,68)$.

The hypocretin neurons send excitatory projections to the entire central nervous system, with the exception of the cerebellum. The excitatory effects on the monoaminergic and 
cholinergic nuclei in the brain stem and hypothalamic regions including norepinephrenic neurons of the locus coeruleus, serotonergic neurons of the dorsal raphe nucleus, histaminergic neurons of the tuberomamillary nucleus and dopaminerigic neurons of the ventral tegmental area, increase arousal and maintains wakefulness. Moreover, hypocretin has excitatory effects on cholinergic neurons of the basal forebrain which is important in maintaining attention and arousal $(67,68)$. Generally, neurons in these cell groups are most active during wakefulness, have decreased activity during NREM sleep, and exhibit no activity during REM sleep (68). The neurons responsible for inhibiting hypocretin neurons are the GABAergic neurons from the venterolateral preoptic area. It is believed that hypocretin functions within a "flip-flop" model, stabilizing wakefulness and preventing aberrant switching between the states. However, this does not explain how the states can simultaneously be present at the same time, such as the intrusion of REM sleep into a wakeful mind (68).

\section{$\underline{\text { Hypocretin and stress }}$}

Hypocretin also plays an important role in stress response as it influences neuroendocrine function by stimulating the hypothalamic-pituitary-adrenal axis (69). This is believed to be mediated by activating corticotropin releasing factor-expressing neurons in the periventricular hypothalamic nucleus and the central nucleus of the amygdala (69). Experimental studies show that activation of hypocretin 1 receptor promotes anxiety-like behavior. This interaction is reciprocal and hypocretin is activated by exposure to various stressors (70).

\section{$\underline{\text { Hypocretin, feeding behavior and energy metabolism }}$}

As mentioned, hypocretin was originally known to increase appetite. This is to some degree mediated by activation of neuropeptide $Y$ neurons in the arcuate nucleus (71). The hypocretin neurons project to the dopaminergic neurons of the ventral tegmental area that are associated with the reward system and to the hypothalamic nuclei that stimulate feeding behavior $(68,71)$. Furthermore, hypocretin seems to be involved in the regulation of energy 
homeostasis. Experimental studies have shown that hypocretin neuron-ablated mice display narcolepsy, hypophagia and obesity. Reduced feeding and obesity may be a result of an underlying reduction in energy expenditure, either due to decreased motor activity and/or a lower basal metabolic rate (72). Intracerebral infusion of hypocretin 1 increases feeding behavior in rats (71), so a decreased hypocretin level would be expected to reduce food consumption and decrease body weight (73). From an evolutionary perspective, when experiencing shortage of food, the hypocretin system will activate, enhancing wakefulness so that one can seek out food (74).

\subsection{Narcolepsy after the influenza A (H1N1) epidemic and vaccination campaign}

\subsubsection{H1N1 epidemic and vaccination campaign}

In 2009 the World Health Organization (WHO) declared a pandemic caused by the influenza A virus H1N1, also known as the "swine flu". This influenza strain resembled the influenza virus responsible for the Spanish flu (1917-1920), which probably caused the death of 50100 million people (75). It was predicted that the virus would cause an epidemic in Norway which would peak around autumn 2009 (76).

Eight different vaccines were licensed internationally. The Pandemrix vaccine, produced by GlacsoSmithKline was the most used vaccine in Europe and also the one recommended to Norwegian citizens. It was an adjuvanted vaccine, which had shown a longer lasting immunity compared to non-adjuvanted vaccines (77). The vaccination started in September 2009, and the order of priority was suggested by WHO and the European Unit Health Security Committee. The groups targeted first were patients with underlying medical conditions, followed by pregnant women, health workers and finally children. Vaccination of these groups started around week 38 in Norway. Vaccination of the rest of the populations started around week 43 . The vaccination coverage in Norway was $41.3 \%$. The percentage of vaccinated children below 5 years of age was $47 \%$. The number of laboratory confirmed H1N1 influenza cases in 2009/2010 season was almost 14000 (approximately 200/100 000). 147 patients were admitted to an intensive care unit, and 32 patients died. In the following season 2010/2011 the incidence of H1N1 influenza cases was just below 30/100 000 . 
The laboratory confirmed H1N1 influenza incidence peaked in week 45, when the cumulative Pandemrix vaccine coverage was just below $10 \%$. Hence, the vaccines were distributed late relative to the epidemic peak (76).

Figure 1. Pandemic vaccination coverage and influenza A (H1N1) cases

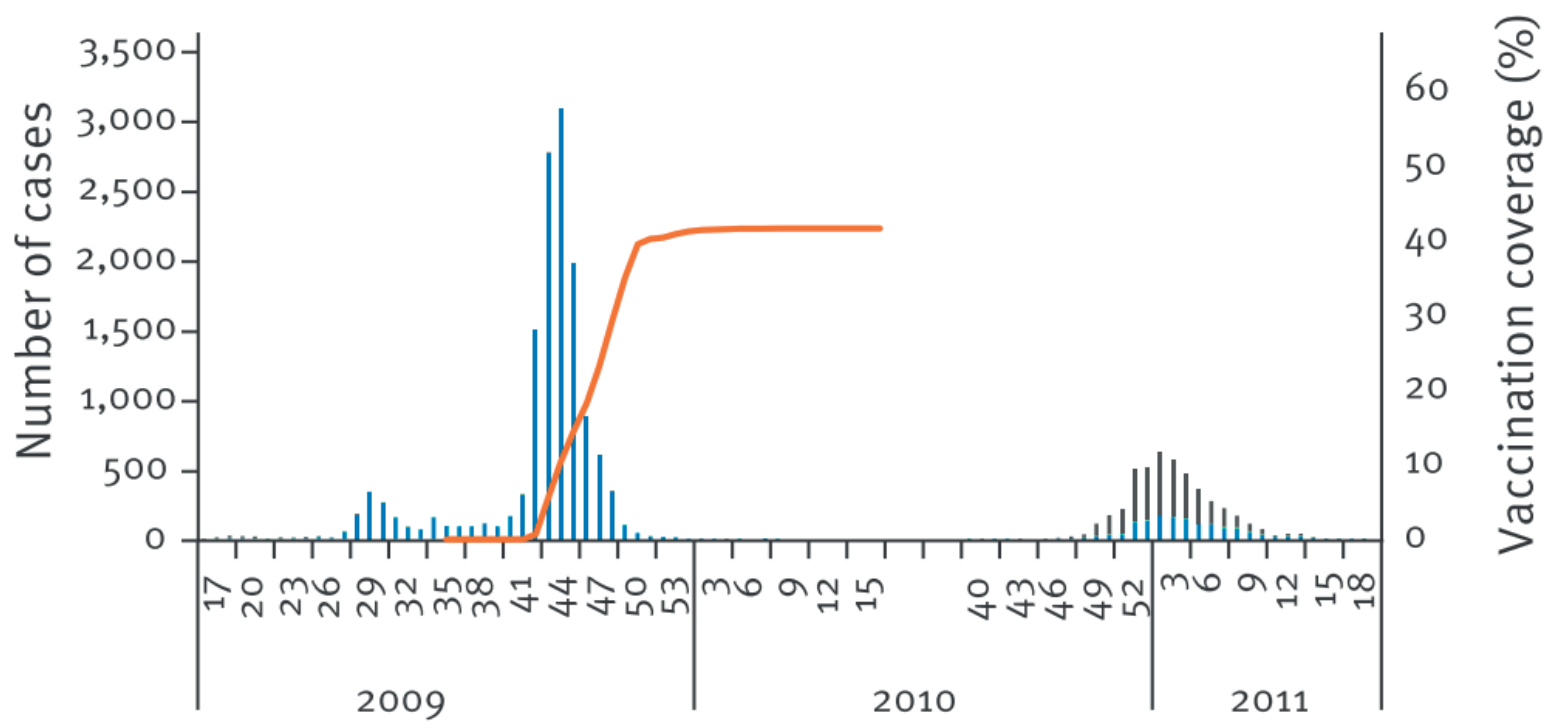

Week and season

\footnotetext{
- Influenza A (H1N1)

- Influenza B

- Vaccine coverage
}

Figure 1 shows the cumulative pandemic vaccination coverage and laboratory-confirmed influenza A (H1N1) and influenza B cases by week, influenza seasons 2009/10 and 2010/11 in Norway. Reprinted with permission from Euro Surveillance (76), Copyright 2016. 


\subsubsection{Post Pandemrix narcolepsy}

The possibility of narcolepsy being a rare side effect of H1N1 flu vaccination was first reported by the Swedish Medical Product agency in August 2010 (78). Additional cases potentially linked to Pandemrix vaccination were reported from Finland and also from sleep centers in Canada, France and America (6). Furthermore, China reported a 3 fold increase in narcolepsy incidence possibly due to the influenza virus itself and not the vaccine since the vaccination coverage was low and only $5.6 \%$ of the patients recalled receiving vaccine (51). The exact mechanism of how the Pandemrix vaccine could cause NT1 is not yet proved (45). However, it has been acknowledged as an adverse effect of the vaccine, and patients have received reimbursement from The Norwegian System of Patient Injury Compensation (NPE) which is a government agency under the Norwegian Ministry of Health and Care Services. Further, it has been speculated if patients with PPN have a different phenotype than those of SN $(5,6)$.

\subsection{Health related quality of life}

\subsubsection{What is health related quality of life?}

WHO defines quality of life as an individual's perception of their position in life in the context of the culture and value systems in which they live and in relation to their goals, expectations, standards and concerns (79). It is a broad ranging concept affected in a complex way by the person's physical health, psychological state, level of independence, social relationships, personal beliefs and their relationship to salient features of their environment. HRQoL considers many different aspects related to a person's perception of quality of life affected by health status. These include physical, psychological, functional and social aspects. Objective physiologic measures provide information to clinicians, but often correlate poorly with functional capacity and well-being, the areas in which patients are most interested. Even more, the subjective experience in a given condition or situation may be dramatically different between patients. The purpose of HRQoL measurement is to evaluate and quantify the degree to which the medical condition or its treatment impacts the individual's life in a valid and reproducible way (79). 


\subsubsection{Health related quality of life in narcolepsy}

Cross-sectional studies have shown impaired HRQoL in patients with narcolepsy in both children and adults as well as in PPN and SN patients $(15,16,80-83)$. There are several factors that may influence HRQoL in patients with narcolepsy. First to be mentioned are the symptoms of narcolepsy themselves, such as EDS and cataplexy. Stores et al. found no significant differences between HRQoL in patients with narcolepsy and those with EDS due to other causes than narcolepsy, and concluded that EDS is the main factor responsible for the impaired HRQoL (84). In contrast, Ozaki et al. found that the severity of subjective sleepiness was not related to the degree of decline of HRQoL (81). Patients with narcolepsy often experience challenges in their education, school performance and social relationships, and they have fewer leisure activities $(82,85)$. As suggested by several authors, the clinical symptoms in addition to the social problems may lead to a decrease in psychosocial function and hence, affect HRQoL $(80,82,85)$. Other factors likely to affect HRQoL in patients with narcolepsy are other coexisting disorders and additional symptoms such as depression (86). Depressive symptoms are common in patients with narcolepsy, and Inocente et al. found depression to be the factor with the greatest impact on HRQoL in patients with NT1 (86).

\subsection{Psychiatric symptoms}

\subsubsection{Sleep and psychiatry}

Psychiatric disorders are associated with sleep disturbances, sleep architecture and the circadian rhythm. They are codependent conditions, and the relationship between psychiatric disorders and sleep disturbances are bidirectional (87).

About two-thirds of patients with depression experience some type of insomnia, including sleep-onset insomnia, frequent awakenings and early morning awakenings. Hypersomnia is reported in 19\% (87). There may be a combination of insomnia and hypersomnia, which is, in children, associated with a more severe depression (87). Interestingly, other sleep abnormalities such as the symptoms sees in narcolepsy have been reported in patients with depression. Szklo-Coxe et al. found a 2.3 fold increase in $\mathrm{HH}, 2.9$ fold increase in SP as well as a 3.2 fold increase in cataplexy-resembling episodes (88). PSG findings in depressed patients show abnormalities in the sleep architecture with reduction in SWS, and an increased 
percentage of REM-sleep. As for anxiety disorders, the percentage of SWS is decreased, and a positive correlation between anxiety severity and number of awakenings has been reported. Furthermore, childhood sleep problems may be a predictor for anxiety disorder in adulthood $(87,89)$.

Patients with bipolar disorders typically have two different sleep patterns when being in a manic phase or when being depressed. In the depressed phase, sleep pattern and PSG findings resemble those of depression. In the manic phase, subjects may feel that 2-4 hours of sleep is sufficient. PSG will show a reduction in total sleep time and a short REM sleep latency. Acute psychosis is often associated with severe sleep problems, and sleep efficiency may be reduced prior to psychosis. Psychotic patients may have problems initiating sleep and may have partially or completely disrupted daily circadian rhythm.

The prevalence of sleep problems in patients with ADHD is reported to be up to 55\%. Various sleep disorders such as periodic leg movement, RLS, insomnia, increased nocturnal motor activity and sleep-breathing disorders are associated with ADHD. Although the causes of ADHD may be multifactorial, it is hypothesized that ADHD in some children is a "night and day disorder", implying that disturbed night contributes to the inattention and hyperactivity (90).

\subsubsection{Narcolepsy and psychiatry}

Narcolepsy was in the very beginning recognized as a psychiatric disorder. Daniels et al. claimed in 1934 that before the onset of the disorder, patients showed neurotic and psychopathic tendencies in their personalities (91). Authors reported a high prevalence of personality disorders, depression, schizophrenic tendencies as well as anger issues (92). In 1976, Roy et al. dismissed that there was a characteristic personality or psychopathology in patients with narcolepsy, but he claimed that the distress of having narcolepsy, including the withdrawal and impaired social life leads to development of personality disorders (92).

The psychiatric aspects of narcolepsy have in the later years received much attention as several more recent studies support the high prevalence of psychiatric disorders in patients with NT1. Depression, anxiety disorders, bipolar disorders, obsessive compulsive disorders, 
eating disorders, ADHD, schizophrenia and even autism spectrum disorders, are among the reported comorbidities $(8,9,93-96)$. However, it should be emphasized that several of the reported comorbidities are based on questionnaires and not a thorough evaluation by a psychiatrist. This does not diminish the importance of the findings, on the contrary, it underlines the relevance of possible symptom overlap and plausible common pathophysiological mechanism.

\subsection{Comorbidities}

\subsubsection{Medical comorbidity}

NT1 patients have a higher prevalence of other concomitant sleep disorders such as OSAS, RLS and REM sleep behavior disorder $(8,97)$. Medical comorbidity, such as disorders of the digestive tract $(8,98)$, nervous system $(97,98)$, upper airway, endocrine disorders, metabolic disorders $(8,97)$ autoimmune disorders $(99)$ and heart disorders $(8)$ are also reported with a higher prevalence in patients with narcolepsy compared to in controls. Studies are, however, divergent (100). The most striking finding is the high prevalence of overweight and obesity which is present in $30-60 \%$ of patients with NT1 $(8,11,12,101)$.

The hypocretin-system is involved in the regulation of autonomic nervous function and may therefore influence sympathetic activity which regulates cardiovascular activity (102). Animal studies have shown an increase in blood pressure and heart rhythm after administration of hypocretin, believed to be due to an excitatory effect on sympathetic activity (103). However, Grimaldi et al. found that patients with narcolepsy had a nightly blood pressure non-dipping pattern indicating an increased sympathetic activity in NT1 patients, despite hypocretin deficiency (104). Nightly blood pressure was associated with increased amount of fragmented sleep. The authors propose that the cardiovascular changes may be a result of not only the hypocretin deficiency and the effect of this on regulatory mechanisms, but also an altered sleep-wake mechanism (104). Jennum et al. report a slightly but not significantly increased mortality rate in patients with NT1 (97). 


\subsubsection{Obesity}

Early case series from 1934 suggested an association between narcolepsy and obesity (91, 105). This has been confirmed in several consecutive studies of both children and adults with narcolepsy $(8,11,12,101)$. Studies of SN patients have shown that early onset of symptoms is associated with obesity $(11,12)$. In addition, an early onset of symptoms has been associated with a greater disease severity. Thus, one may speculate whether obese patients also have a more severe narcolepsy (37).

As described in more detailed in paragraph 1.1.8, the hypocretin neuropeptide itself is known to stimulate appetite, and in experimental studies, central administration of hypocretin 1 increases food intake (71). It is therefore rather paradoxical that hypocretin deficient patients have a tendency to be obese (73). Furthermore, the increase in weight is despite a reduced daily intake of calories compared to controls (106).

Appetite may be increased as a result of decreased serum level of leptin, which is an appetite-suppressing hormone. Decreased leptin-binding in the hypothalamus may lead to disinhibition of neuropeptide $Y$, which is a potent appetite stimulant $(107,108)$. Obesity may also be the results of circumstances related to narcolepsy symptoms, such as EDS that may result in reduced energy expenditure. This is supported by the fact that other sleep disorders also have a higher prevalence of obesity $(12,109,110)$. Eating can be a coping mechanism in an attempt to try to stay awake which may be a reason why narcolepsy patients tend to choose food with high percentage of carbohydrates and calories $(111,112)$.

\subsubsection{Fatigue}

Fatigue may be defined as a subjective experience of mental or physical exhaustion that does not disappear after a period of sleep (14). Differentiating between fatigue and sleepiness may be challenging, in particular in children (86). Individuals with fatigue demonstrate sleepiness, and vice versa implying that there may be a shared biological basis between fatigue and sleepiness, or that there might be overlap of symptoms (113). Although further documentation is still needed, there are studies showing that the majority of patients with narcolepsy suffer from severe fatigue $(14,86,114)$. Individuals with 
hypersomnia of other causes than narcolepsy also demonstrate substantial fatigue, suggesting that the sleepiness itself causes fatigue (113).

\subsection{Treatment}

There is not yet any cure for narcolepsy. Pharmacological treatment, as well as nonpharmacological treatment, is based on relieving various narcolepsy symptoms, such as EDS, cataplexy, hypnagogic hallucinations and fragmented sleep (30). A combination of medications is often needed to address the various symptoms. Experimental immunemodulating therapy with immunoglobulins has shown inconclusive results $(115,116)$. Among promising future medical treatment is hypocretin replacement therapy, which would be the ultimate gold standard. However, the blood-brain barrier seems to be impermeable to hypocretin 1. Experimental studies are still in process (117). Table 2 lists the most common medications used in the treatment of NT1.

\section{$\underline{\text { Non-medical treatment }}$}

Most patients, either medicated or non-medicated, benefits from a regular and consistent sleep hygiene and taking short naps during the day (118). A multidisciplinary approach is important and should address problems concerning school performance, psychosocial distress and comorbidity including psychiatric and nutritionist evaluations $(119,120)$. 


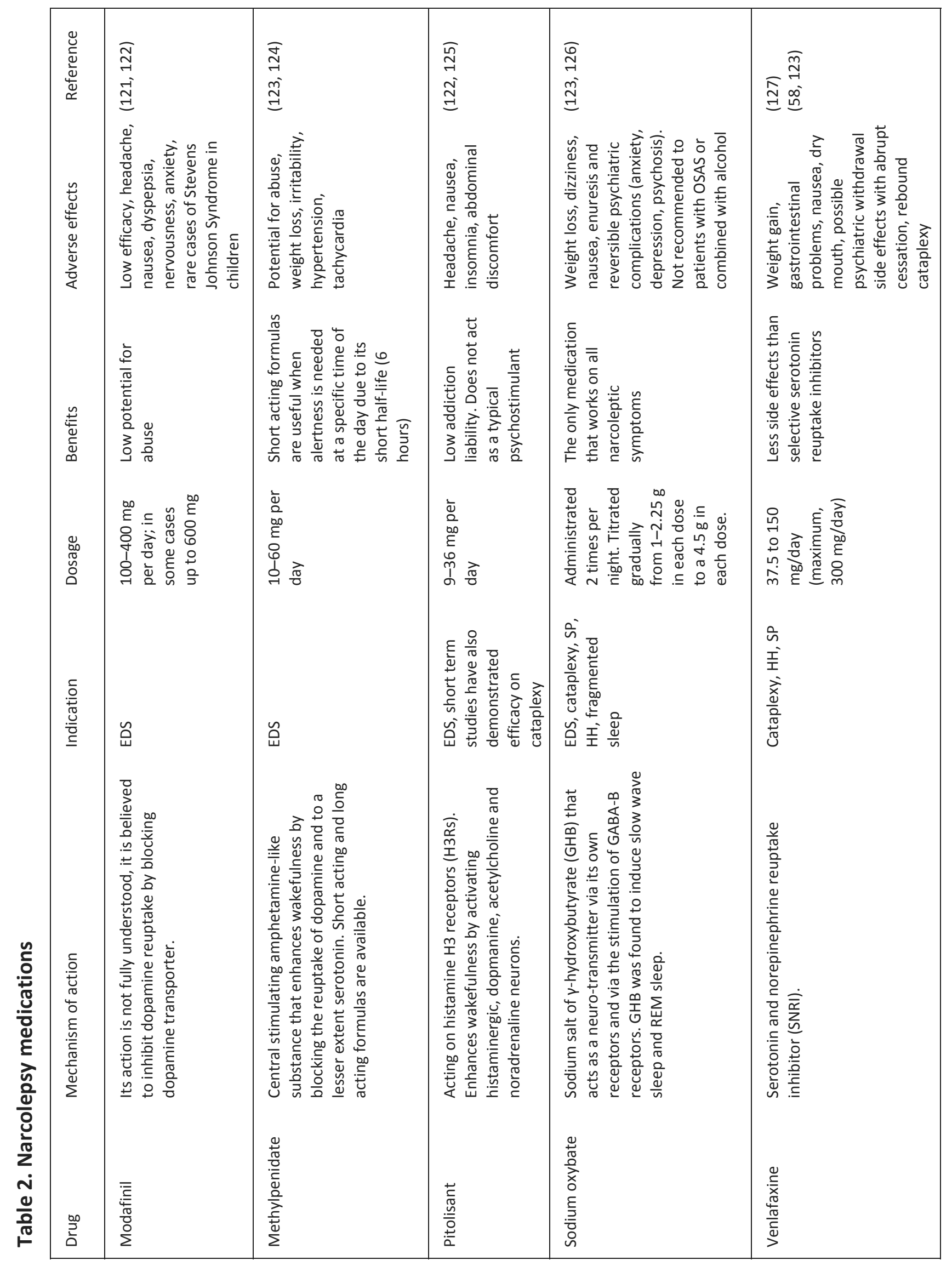




\section{THESIS AIMS}

The aim of this thesis was to characterize the phenotype in patients who developed NT1 after the H1N1 influenza epidemic and vaccination campaign in 2009 in Norway, primarily focusing on HRQoL, psychiatric symptoms and medical comorbidities (overweight /obesity in particular). Further, we aimed to explore factors that could be associated with HRQoL and changes in HRQoL (Paper I), psychiatric symptoms (Paper II) and overweight/obesity (Paper III).

Paper I

The primary aim of the study was to prospectively study changes in HRQoL over time in a Norwegian cohort of mainly post-H1N1 vaccination NT1 patients. The secondary aim was to investigate possible predefined predictors for changes in HRQoL; EDS, level of psychiatric symptoms and the use of sodium oxybate.

Paper II

The primary aim of the study was to explore the extent and characteristics of psychiatric symptoms among children and young adults who developed NT1 after the H1N1 influenza epidemic and vaccination campaign in 2009 in Norway. We hypothesized a high prevalence of psychiatric symptoms. We further wished to explore possible explanations for the high prevalence of psychiatric symptoms. There may be symptom overlap, psychiatric symptoms may be secondary to the narcolepsy symptoms, EDS in particular or NT1 and psychiatric symptoms may share common pathophysiological mechanisms.

\section{Paper III}

The primary aim of the study was to study the extent of overweight and obesity and other medical comorbidities among Norwegian patients who developed NT1 after the 2009 H1N1 influenza epidemic and vaccination campaign. We also wanted to explore possible 
associations between overweight/obesity and the following factors: age at disease onset, disease duration, various indicators of severity of narcolepsy (cataplexy, sleepiness,

fragmented sleep, hypocretin 1 levels), and additional comorbidities (psychiatric symptoms, medical comorbidities, fatigue). 


\section{MATERIALS AND METHODS}

\subsection{Study design}

The three studies included in the PhD thesis are parts of a larger national project called "Phenotype and pathogenesis of narcolepsy following H1N1-vaccination in Norway". Paper I; "Changes in quality of life in individuals with narcolepsy type 1 after the H1N1-influenza epidemic and vaccination campaign in Norway: a two-year prospective cohort study", was a prospective observational study where the main outcome was to evaluate changes in HRQoL over time. The two following papers, "Psychiatric symptoms in patients with post-H1N1 narcolepsy type 1 in Norway" (Paper II) and "Obesity, other medical comorbidities and fatigue among NT1 patients after the Norwegian H1N1 influenza epidemic and vaccination campaign" (Paper III), were observational studies with cross-sectional design.

\subsection{Setting}

The studies were carried out at the Norwegian Center of Expertise for Neurodevelopmental Disorders and Hypersomnias (NevSom), Oslo University Hospital (OUH), Ullevål, between February 2015 and September 2017. NevSom is an expertise center, which was delegated a central role in surveying patients who developed narcolepsy after the H1N1 influenza epidemic and vaccination campaign autumn 2009 by the Norwegian Ministry of Health and Care Services. Patients diagnosed with narcolepsy after the H1N1 influenza epidemic and vaccination campaign, were reported to NevSom either by direct reports from hospitals and clinicians or by the results from hypocretin 1 measurements. Patients were recruited by telephone calls, after receiving a letter with study information and a battery of questionnaires in advance. The majority of the patients had CSF collected by lumbar puncture for hypocretin measurements at their local hospital prior to inclusion. The hypocretin 1 measurements were performed at the Hormone Laboratory at OUH, Aker, with the method described by the producer, Phoenix Pharmaceutical St. Joseph, MO, USA (128). Clinical examinations, sleep investigations and blood samples were performed at NevSom. Semi-structured interviews were conducted in relation to inclusion. Baseline questionnaires used for Paper I were collected either by returning the questionnaire by post or handling 
them personal to NevSom. Follow up data for Paper I was collected through telephone interviews.

\subsection{Study sample and participants}

The project's inclusion criteria were: age 5-99 years, onset of narcolepsy symptoms after autumn 2009 and narcolepsy diagnosis based on the ICSD-3 (1).

In December 2014, 91 individuals identified as our national cohort of narcolepsy patients with onset after autumn 2009 were invited to participate in the study and were sent baseline questionnaires. During the inclusion period, an additional 47 individuals were made known to us by one of the ways described above. Of the 138 eligible participants, 38 individuals chose not to participate. Four individuals were excluded because they did not fulfill the diagnostic criteria for NT1. Five individuals were excluded because they had onset of symptoms before 2009. One of these five patients had typical core narcolepsy symptoms, but we discovered that she had had debut of symptoms immediately before vaccination with Pandemrix. She was included in Paper I, but excluded from the two following studies.

During the first quarter of 2015, 42 of the 91 patients who had received the baseline questionnaires in 2014 returned them. Only 36 of the returned questionnaires were complete, and three of the participants that had returned the questionnaire were among the participants excluded as described above (see also Figure 2 below). A total of 76 participants answered the questionnaire concerning psychiatric symptoms (Paper II). 


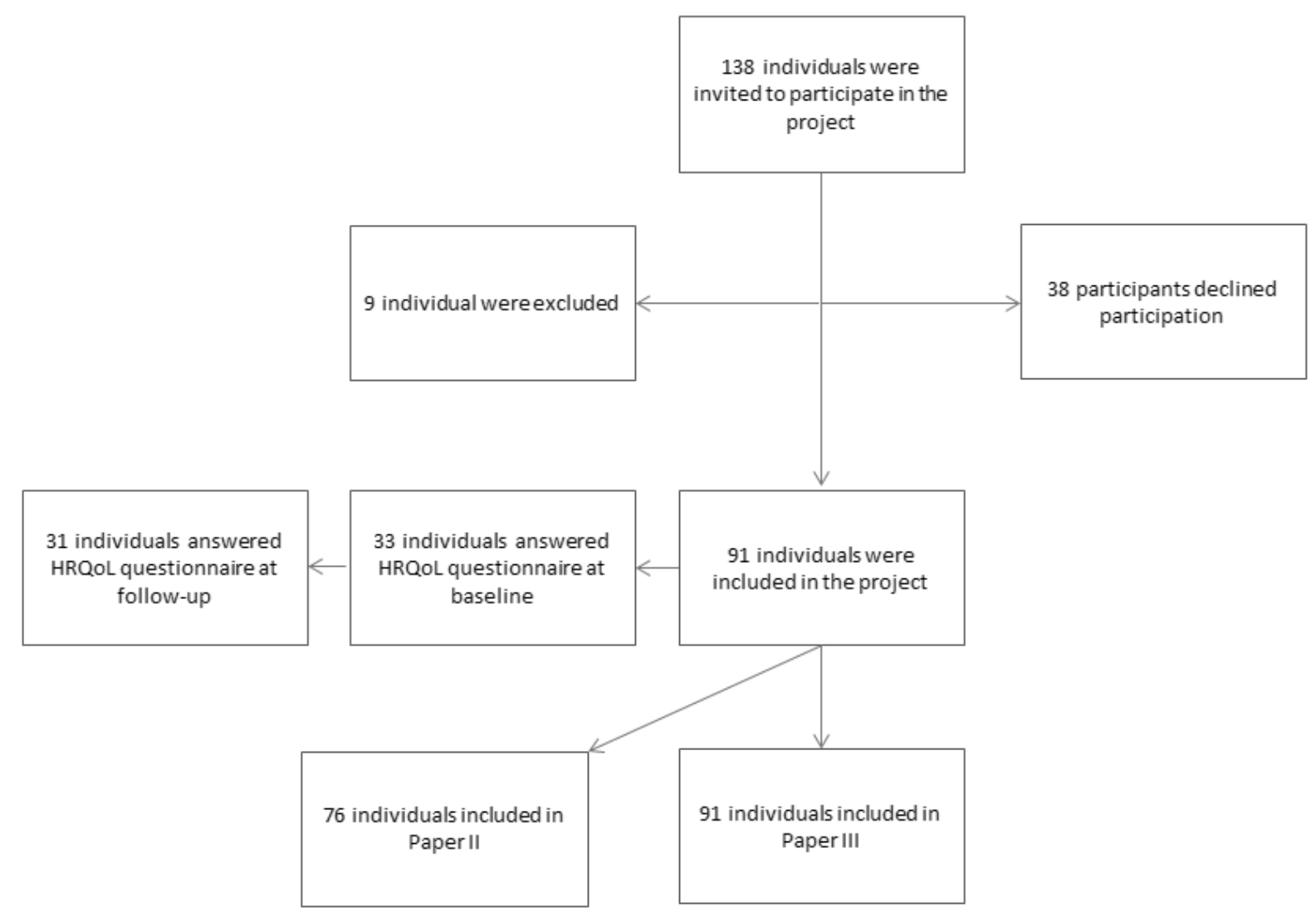

* One of the nine individuals excluded from the project was included in Paper I.

\subsection{Sleep investigations and scoring}

\section{Sleep investigation}

Patient's circadian rhythm was monitored with actigraphs (Actiwatch Spectrum Plus, Phillips) for 10-14 days prior to the sleep investigations. For the overnight PSG recording, patients slept at the at Ullevål Hospital Hotel. PSG recordings were assessed with the SOMNOmedics system (SOMNOmedics $\mathrm{GmbH}$, Randersacker, Germany) according to the recommendations of the American Academy of Sleep Medicine (AASM) Manual for the scoring of sleep and associated events: rules, terminology and technical specifications version (129). The impedance was controlled before sleeping and adjusted if necessary as we aimed to keep the impedance beneath $5 \Omega$. The overnight PSG recording was followed by MSLT which was carried out at the sleep laboratory at NevSom. Patients were given five nap opportunities of 
30 minutes with two hours interval. The same equipment was used in the MSLT recordings as in PSG recordings, except that the nasal thermistor, nasal pressure transducer and respiratory belts for measuring respiratory effort, were removed for the MSLT recordings. Impedance was controlled before each MSLT.

\section{Sleep scoring}

Sleep stage and associated events were scored according to the AASM (129). All patients (except for one case where venlafaxine was continued due to severe cataplexy) ceased narcolepsy medication 10-14 days prior to inclusion. Table 2 lists the relevant sleep events and their definitions for the papers in this thesis.

Table 2. Sleep events

\begin{tabular}{|l|l|}
\hline Sleep event & Definition \\
\hline Total sleep time (TST)(hours) & $\begin{array}{l}\text { Period of time in bed between light off and light } \\
\text { on markers excluding the wake stage }\end{array}$ \\
\hline Apnea hypopnea index (AHI) & $\begin{array}{l}\text { Number of apneas and hypopneas events } \\
\text { divided by TST }\end{array}$ \\
\hline Awakening index & Number of wake periods divided by TST \\
\hline Sleep stage change index & Number of sleep stage changes divided by TST \\
\hline SOREM & $\begin{array}{l}\text { At least one epoch of REM sleep within the first } \\
15 \text { minutes after sleep onset. }\end{array}$ \\
\hline
\end{tabular}




\subsection{Anthropometric measurements and blood pressure}

\section{Performance}

Patient's weight was measured wearing light clothing using a portable Konig Electronic HCPS500 scale. Height was measured using a Seca (CE0123) stadiometer (Vogel \& Halke, Hamburg, Germany) to the nearest $0.5 \mathrm{~cm}$. Waist circumference was measured at the midpoint between the upper part of the iliac crest and the lower part of the lateral rib cage using a standard anthropometric tape. Blood pressure was measured using a portable blood pressure monitor (US-1020 Digital Blood pressure monitor, A\&D Medical, San Jose, CA, USA). Patients had rested for five minutes and blood pressure was measured three times with one minute intervals.

\section{Calculations and definitions}

We used the standard calculation for body mass index $\left(B M I=k g / \mathrm{m}^{2}\right)$. In adults BMI was classified according to definitions set by the International Obesity Task Force (IOTF) (130); normal weight BMI from 18.5-24.9; overweight BMI from 25-29.9; obesity class I BMI from 30.0-34.9; obesity class II BMI from 35.0-39.9; obesity class III BMI $\geq 40 \mathrm{~kg} / \mathrm{m}^{2}$. For patients younger than 18 years, the BMI was converted to the age and sex adjusted children's BMI SD score (BMI z-score) using the formula from the IOTF (130). BMI in children (iso-BMI) was defined by the BMI value that corresponded to the IOTF threshold for normal weight, overweight (IOTF-25), obesity (IOTF-30) and morbid obesity (IOTF-35) for the child's age and sex.

We calculated the waist-to-height ratio by the formula waist/height. Waist-height ratio was classified according to the cut-off of 0.5 set by the WHO. Waist-to-height ratios below the cut-off were considered normal, and ratios at or above cut-off were considered to indicate abdominal obesity.

We used the mean blood pressure value, and hypertension was classified according to The Task Force for the Management of Arterial Hypertension of the European Society of Hypertension and the European Society of Cardiology (131). For study participants younger 
than 20 years, mean systolic and diastolic blood pressures were converted to age and sex adjusted z-scores with respect to a reference distribution (132).

\subsection{Questionnaires}

\section{Stanford Sleep Questionnaire}

We used the Stanford Sleep Questionnaire (SSQ) to evaluate severity of sleep symptoms. The English version was translated to Norwegian through the well-established forwardtranslations and back-translations (133). Two native Norwegian physicians (Hilde Therese Juvodden and the candidate) performed the forward translation and the back-translation was performed by a professional translator.

\section{Medical comorbidity}

Medical history was obtained by semi-structured interviews using a comprehensive list of disorders.

\section{$\underline{\text { Health related quality of life survey }}$}

To assess HRQoL, we used the Norwegian version of Pediatric Quality of Life ${ }^{\mathrm{TM}}$ Generic Core Scales 4.0 (PedsQL). We used the self-reported versions that are available for different age groups where items are age appropriate and essentially identical. The PedsQL is a 23-item questionnaires which can be divided into four functioning scales; 1) physical (eight items); 2) emotional (five items); 3) social (five items); and 4) school (five items) (134). Each item is scored on a five-point response scale, referring to how much of a problem each item has been during the past month $(0=$ never a problem, $1=$ almost never a problem, $2=$ sometimes a problem, 3 = often a problem, $4=$ almost always a problem). Items are reverse-scored and linearly transformed to a 0 to 100 scale $(0=100,1=75,2=50,3=25,4$ $=0$ ), and higher scores indicate better HRQoL. The scores were computed according to the scoring instruction manual (135). 


\section{Fatigue survey}

To assess fatigue we used the Norwegian version of the Pediatric Quality of Life Multidimensional Fatigue Scale (PedsQL Multidimensional Fatigue Scale). We used selfreported versions that are available for different age groups where items are age appropriate and essentially identical. PedsQL Multidimensional Fatigue Scale is an 18itemquestionnaire which can be divided into three subscales, each subscale containing 6 items; 1) general fatigue; 2) sleep/rest fatigue; and 3) cognitive fatigue. The scoring instructions are similar to those of the PedsQL described above and were computed according to the instruction manual (136).

\section{$\underline{\text { Psychiatric symptoms survey }}$}

To assess psychiatric symptoms, we used the Norwegian version of the Achenbach System of Empirically Based Assessment (ASEBA) Child Behavior Checklist (CBCL) for patients aged 618 years and the corresponding Adult Self Report (ASR) for patients aged 18-59 years. The CBCL consists of 120 items in eight syndrome scales: anxious/depressed, withdrawn/depressed, somatic complaints, social problems, thought problems, attention problems, rule-breaking behavior, and aggressive behavior. The ASR and CBCL are equivalent, with the exception that in the ASR the social problem syndrome scale is replaced by that of intrusive behavior. Each item is scored on a three-point response scale $(0=$ not true, 1 = somewhat or sometimes true, and 2 = very often true) which are then added to compute a sum score.

The scores from the syndrome scales; anxious/depressed, withdrawn/depressed and somatic complaints are used to derive an internalizing symptoms score. The scores from the syndrome scales; rule-breaking behavior, aggressive behavior and intrusive behavior (adults only) are used to derive an externalizing symptoms score. A total psychiatric symptoms score is derived by all the syndrome scales. The questionnaires were manually scored, and the raw scores were converted into T-scores to enable comparison. In order to assess if patients had problems of clinical concern, scores were measured against percentiles from a normative non-referred population. 


\subsection{Statistics}

All statistical analyzes were performed with IBM SPSS version 23 (Paper I and II) and version 25 (Paper III). Data were presented as frequencies, means with standard deviation (SD) or 95\% confidence intervals $(\mathrm{Cl})$ or medians with interquartile ranges (IQR), as appropriate. Values of $p<0.05$ were considered statistically significant.

Paper I

In Paper I, we used paired sample t-tests to assess changes between PedsQL total health summary score at baseline and follow-up. We calculated the mean PedsQL total health summary score at baseline and divided the patients into two groups based on whether their scores were below or above the average score at baseline. We further used an independent sample t-test to assess which group had improved their scores. Multiple linear regression analysis, adjusted for age and gender, was used to assess associations between explanatory variables (sodium oxybate, psychiatric symptoms and EDS) and changes in HRQoL.

Paper II

In Paper II, we used a multiple linear regression analysis, adjusted for age and gender to assess associations between the explanatory variables (mean sleep latency in MSLT, sleep stage switch index, awakening index and CSF hypocretin 1 concentration (pg/ml)) and the outcome of psychiatric symptoms.

\section{Paper III}

Normal weight was defined as $\mathrm{BMI}<25$ and overweight/obesity $\mathrm{BMI}$ as $\geq 25$, to allow for statistical comparison. We used separate linear regression analyses to assess associations between this variable and outcomes of age at disease onset and disease duration and BMI. We used multiple regression analysis to assess associations between measures of disease severity and BMI. The following variables were used to represent disease severity: 1) mean frequency of cataplexy, 2) MSLT (min, sec), 3) mean sleep stage change index (number of 
changes/total sleep time) and 4) CSF hypocretin 1 concentration ( $\mathrm{pg} / \mathrm{ml})$. Multiple regression analysis was also used to assess associations between comorbidities and BMI. The following variables were used to represent comorbidities 1) presence or absence of medical comorbidity (categorical variable), 2) psychiatric symptoms (mean total ASEBA score), 3) fatigue (mean total fatigue score). 


\section{SUMMARY OF RESULTS}

In this thesis, characterizing the phenotype of patients who developed NT1 after the H1N1 epidemic and vaccination campaign, we show that NT1 is a heterogeneous disorder complicated with several additional symptoms and comorbidities. Our findings provide insight into the complexity of the disorder and hopefully point to aspect of the disorders clinicians should address in the treatment and follow-up of patients.

\section{Paper I}

HRQoL is measured in different domains that together render a total perspective on a patient's subjective view on health related aspects of life. We found a markedly impaired HRQoL in all domains when comparing our results to national normative data. The domains of school/work functioning and emotional functioning showed the greatest impairments, both at baseline and at follow up. Little has previously been documented about changes in HRQoL in NT1 over time, and in which domains changes are likely to be seen. We found that the mean HRQoL's total summary score significantly improved from baseline to follow up by 5.9 points $(95 \% \mathrm{Cl}: 0.4,11.4)$. The largest improvements were seen in the domains of physical functioning (improved from mean (SD) score of 65.9 (20.7) to 75.7 (17.4)), and in school functioning (improved from mean (SD) score of 53.4 (18.4) to 60.8 (13.7)).

We further explored three factors that, based on previous literature and clinical experience, were likely to affect changes in HRQoL. The potential predictors we used were EDS (measured by ESS), psychiatric symptoms (measured by ASEBA) and use of sodium oxybate at follow-up. Neither EDS nor psychiatric symptoms were associated with improvement seen in HRQoL's total summary score. However, we found that the use of sodium oxybate at follow up was significantly associated with changes in HRQoL $(p=0.027)$, when adjusted for age and gender. Although psychiatric symptoms were not associated with changes in HRQoL, we found that $31 \%$ of the patients had psychiatric symptoms total scores in the clinical range of concern indicating a high level of psychiatric comorbidity. 
Paper II

Our first study (Paper I), confirmed the high prevalence of psychiatric symptoms in patients with NT1. We therefore wanted to further explore the extent and characteristics of psychiatric symptoms.

The cohort included children and adults, which enabled us to explore possible similarities or dissimilarities between the two groups in regard to psychiatric symptoms. We found that internalizing symptoms were the most prevalent psychiatric symptoms in both children and adults. A total of $38 \%$ of children and $33 \%$ of adults had internalizing symptoms in the clinical range of concern. These internalizing symptoms were mainly driven by somatic complaint in both children and adults, followed by withdrawn/depression in children and anxiety/depression in adults. Further, we found that thought problems were prevalent in children with $32 \%$ of children having thought problems in the clinical range of concern. Attention problems were more prevalent in adults than in children with $28 \%$ of adults reporting problems in the clinical range of concern in contrast to $8 \%$ in children.

Even though an association between psychiatric symptoms and NT1 is established, this association still needs further examination. In order to assess overlap of psychiatric symptoms and narcolepsy symptoms, we tested the effect of removing items concerning sleep/core narcolepsy symptoms from the psychiatric symptom questionnaire. We found that although the psychiatric symptoms total scores were then lower, still $27 \%$ of children and $22 \%$ of adults had symptoms in the clinical range of concern (versus $30 \%$ and $25 \%$ respectively, with sleep/core narcolepsy symptoms items included). This suggests that the association between psychiatric symptoms and narcolepsy is not just due to symptoms overlap.

We found no significant association between the summary scores of psychiatric symptoms and daytime sleepiness (measured by MSLT and ESS), or with the level of hypocretin 1 in CSF in neither children nor adults (all $p>0.12$ ). In children, fragmented sleep, measured by sleep stage change index, was significantly negatively associated with internalizing, externalizing and total summary scores (all $p \leq 0.020$ ), and awakening index was negatively associated with externalizing and total summary scores (both $p=0.042$ ). In adults, sleep stage change 
index was not associated with either of the summary scores (all $p>0.09$ ), but awakening index was positively associated with internalizing scores $(p=0.015)$.

\section{Paper III}

It is not known whether some NT1 patients have a more severe form or a more "full blown" version of narcolepsy that includes additional comorbidities. In this study, we explored the prevalence of medical comorbidities, overweight/obesity and fatigue. We further explored the possibility that patients with overweight/obesity had a more severe form of narcolepsy. Results showed that some form of medical comorbidity was present in $51 \%$ of the patients. Autoimmune disorders were found in $7 \%$, allergic disorder in $35 \%$ and psoriasis in $5 \%$. In children, $54 \%$ were overweight or obese ( $70 \%$ of the girls and $40 \%$ of the boys), and girls were significantly more obese than boys $(p=0.049)$. In adults, $61 \%$ were classified as overweight or obese ( $58 \%$ of the females and $70 \%$ of the males).

The severity of fatigue was measured on a scale from 0-100 where lower scores indicate more fatigue. The mean (SD) total fatigue score in children was 50 (17) and the mean (SD) total fatigue scores in adults was 39 (16). The total fatigue scores were significantly lower in adults compared to children $(p=0.015)$.

Based on previous literature and clinical experience we explored factors that could be associated with overweight/obesity. First; age at disease onset and disease duration. Second; measures of narcolepsy symptoms severity (frequency of cataplexy, mean MSLT, fragmented sleep and hypocretin 1 levels in CSF). Third; comorbidities (psychiatric symptoms, medical comorbidities and fatigue). Neither age at disease onset, disease duration or measures of narcolepsy symptoms severity was associated with BMI in children or adults. However, we did find that subjectively reported sleepiness measured by the ESS was significantly positively associated with BMI in children $(p=0.019)$, but not in adults when tested in a separate linear regression analysis. We also found a significant association between BMI and medical comorbidity in children ( $p=0.032)$. 


\section{DISCUSSION}

\subsection{Main findings}

In this chapter, the main findings from the papers included in this thesis will be discussed. Here is a brief summary:

- HRQoL was markedly impaired but improved after 2 years of follow up. The use of sodium oxybate was significantly associated with the improvement in HRQoL.

- There was a high prevalence of psychiatric symptoms, internalizing symptoms in particular. There were no significant associations between EDS, measured by EES and MSLT, and psychiatric symptoms. Sleep stage shift index and awakening index were significantly negatively associated with psychiatric symptoms in children, and awakening index was significantly positively associated with internalizing symptoms in adults.

- Medical comorbidity in general was prevalent, but the prevalence of each single medical disorder was not notably increased compared to the general population.

- There was a high prevalence of overweigh and obesity. The presence of one or several medical comorbidities was associated with overweight/obesity in children, but not in adults.

- Children and adults reported severe fatigue.

- Hypocretin was not associated with either psychiatric symptoms or overweight/obesity.

- No marked differences were found concerning HRQoL, psychiatric symptoms, medical comorbidity, overweight/obesity and fatigue compared to previous literature on SN patients. 


\subsection{Health related quality of life}

In line with our study (Paper I), several cross-sectional studies show an impaired HRQoL in patients with NT1 $(15,16,80-83)$. A previous Norwegian study by Ervik et al. from 2006, reported particularly low scores (poorer functioning) in the domains of general health, bodily pain and social function (137). Poorer leisure activities, absenteeism from school and work, personal conflicts and withdrawal as a consequence of fearing cataplexy are all factors that potentially decrease HRQoL $(82,138,139)$. Ozaki et al. found, when comparing drug naive patients with narcolepsy and cataplexy, narcolepsy without cataplexy and idiopathic hypersomnia, that the degree of subjective sleepiness was not associated with any HRQoL domains in the total patient group, suggesting that the severity of subjective sleepiness does not act as a main contributor to the decrease in HRQoL (81). We found that psychiatric symptoms were inversely correlated to HRQoL at baseline, which is in line with several crosssectional studies as well as the study of Inocente et al. who found that depression was the factor that affected HRQoL the most (82). The increase in HRQoL after 2 years was, however, not associated with psychiatric symptoms at baseline.

Successful treatment of narcolepsy requires individual tailoring of therapy and regular follow-up to monitor response to treatment and potential comorbidities (140). The potential effect of the thorough evaluation of narcolepsy symptoms, information and education about the disorder, individual treatment, including evaluation of psychiatric symptoms and medical comorbidity and assistance in school and work difficulties that all patients received at inclusion, may be a significant, contributing factor to the improved HRQoL seen at follow-up in our study.

We found that sodium oxybate was significantly associated with increased HRQoL at follow up. In support of our finding, Rocca et al. reported that patients treated with sodium oxybate had significantly better school functioning than drug-naive NT1 patients (141). Sodium oxybate stabilizes sleep architecture by increasing sleep latency, decreasing total sleep time and reducing nocturnal awakenings (142), and is the only medications that improves all narcoleptic symptoms (142-144). Sodium oxybate is gradually titrated up to an effective level, and the maximum effect may not be reached until several months after treatment is initiated (145). It is therefore possible that patients that used sodium oxybate at baseline responded better to the medication at follow up. 


\subsection{Psychiatric symptoms}

In Paper II, we found that $38 \%$ of children and $33 \%$ of adults had internalizing psychiatric symptoms in the clinical range of concern. Internalizing score is derived by the subscales; anxiety/depression, withdrawn/depression and somatic complaints. The subscale somatic complaints had the highest scores for both children and adults which is in agreement with previous studies $(85,141)$. Somatic complaints is a descriptive term where the etiology behind the symptoms is not established (146). It is often seen in patients with psychiatric disorders such as anxiety and depression (147) and is inversely related to well-being (146). Neither discomfort nor pain has previously been considered as main symptoms of the narcoleptic disorder $(10,148)$, although Dauvilliers et al. demonstrated that one-third of patients with NT1 experience pain at least monthly, with symptom frequency significantly higher than in controls (149).

A high prevalence of depressive symptoms in patients with narcolepsy is well established although the cause is disputed $(8,84,86,95,150)$. The pathophysiology of narcolepsy itself may be expressed as both depression and core narcolepsy symptoms, as suggested by Broughton et al. as early as in 1981 (151). Alternatively, depression might come secondarily to narcolepsy symptoms and/or to the burden of chronic disorder (141). By using diagnostic tools, Fortuyn et al. found that anxiety and panic attacks were more prevalent than depression in patients with NT1 (9). Patients with narcolepsy may experience lack of control due to sudden sleep attacks, cataplexy, automatic behavior as well as hallucinations. These are stress-inducing situations potentially leading to anxiety and social phobia. Moreover, withdrawal and absenteeism from school and social gatherings may be used as avoiding strategies (9). Interestingly, we found a higher percentage in adults than in children in the clinical range for concern in the subscale anxiety/depressed. Vice versa, children scored higher in the subscale withdrawn/depressed than adults. These finding may imply that the same problems are expressed differently in children then in adults.

Externalizing psychiatric symptoms, that is, maladaptive behavior towards an individual's environment are not uncommon in NT1, and the prevalence of ADHD symptoms is approximately twofold higher in patients with narcolepsy than in the general population (94, 96). It is believed that the symptoms of ADHD, paradoxically, are closely related to hypersomnia, and that the hypervigilance is a coping mechanism in order to sustain 
alertness (96). Hence, there may be a common pathophysiological mechanism. Treatment with stimulants will increase alertness, thereby diminishing the symptoms of inattention and hyperactivity (96). Szakacs et al. found that seven of nine patients with ADHD developed ADHD after the age of 7 years and after narcolepsy onset. They argue a relationship between the onset of narcolepsy and the development of ADHD (95).

Sleep disturbances are part of the diagnostic criteria for several psychiatric disorders, and symptoms of narcolepsy and those of psychiatric disorders may overlap (147). We found that the percentage of patients with psychiatric symptoms total score in the clinical range of concern remained high, even when sleep items were excluded from the analyses. Bearing in mind the disabling symptoms of narcolepsy, it is likely that the burden of the disorder itself may cause psychosocial distress.

We found no associations between EDS, measured by ESS or MSLT and psychiatric symptoms in neither children nor adults. Other studies have reported similar psychiatric symptom load in healthy children with increased daytime sleepiness and in patients with EDS of other causes than narcolepsy, indicating that EDS contributes to psychiatric problems $(84,152)$. Very surprisingly, there was a significant negative association between fragmented sleep and psychiatric symptoms in children, indicating that the more fragmented sleep, the less psychiatric symptoms. In adults, the results were opposite and fragmented sleep measured by awakening index was positively associated with the psychiatric symptoms internalizing score. The latter finding is in line with studies indicating that behavior problems may be associated with sleep problems such as fragmented sleep $(87,153)$. It is, however, possible that children adapt better to fragmented sleep, and Liu et al. reported that sleep fragmentation is not that uncommon in children (41). OSAS is another sleep disorder associated with frequent awakenings and Lewin et al. found that children with OSAS had more behavioral problems than controls, but that children with mild OSAS had more behavioral and emotional problems than children with severe OSAS (40). Moreover, children's psychiatric symptoms in Paper II were rated by their parents contrary to selfreporting of psychiatric symptoms in the adult population of our cohort. Parents may misinterpret children's symptoms, and some parents may focus on their children's narcolepsy symptoms while others focus on the children's psychiatric symptoms. 


\subsection{Medical comorbidity}

The overall prevalence of patients in our cohort having medical comorbidity was $51 \%$, and the most frequent coexisting disorders were allergic disorder (35\%). In the study of Ohayon et al., $27.5 \%$ of patients with NT1 had upper airways disorder, which Ohayon et al. proposed indicated a compromised immune system (8). This is interesting, since it is believed that NT1 is autoimmune mediated, and prior infections with upper airway diseases like Streptococcus pyogenes (58) and H1N1 influenza (51) have been associated with the debut of NT1. Moreover, we found that almost $7 \%$ of our cohort had a disorder of clear autoimmune origin, while the overall estimated prevalence is $4.5 \%$ in the general population (154). Even though studies are divergent, there are reports of a high prevalence of autoimmune disorders in patients with SN $(99,100)$. Supporting these reports, is the theory of shared autoimmunity, implying that one autoimmune disorder is often accompanied by another autoimmune disorder (99).

\subsection{Obesity}

In Paper III, we found that $54 \%$ of the pediatric NT1 patients and $61 \%$ of the adult NT1 patients were overweight or obese confirming the high prevalence of overweight and obesity in patients with NT1 reported by others $(8,11,12,101)$. A logical explanation would be the involvement of the hypocretin-system as experimental studies show that hypocretin regulates feeding behavior and energy homeostasis. This is discussed further in chapter 5.7.

It is possible that sleepiness per se could contribute to increased weight, as the prevalence of obesity is known to be high also in other sleep disorders such as NT2 (12), RLS (110) and OSAS (109). We found that subjective sleepiness was significantly associated with BMI in children, but not in adults. However, we found no associations between objective daytime sleepiness, measured by MSLT, or night fragmented sleep and BMI. This is in agreement with the study of Inocente et al., who found that the severity of sleepiness was high, but without any difference between obese and non-obese groups (12).

In Paper III, neither age at disease onset nor disease duration was associated with BMI in children or in adults. Increase in weight has been observed close up to the onset of narcolepsy symptoms and is reported in some cases to be one of the first symptoms $(11,12$, 
155). Some studies have found that early onset of $S N$ is associated with greater obesity (11, 12). Although there can be a rapid increase in weight around the time of disease onset, BMI seems to stabilize gradually after $3-4$ years $(11,155)$. It is possible that our cohort differs from previous cohorts of mainly SN patients in that patients in our cohort was diagnosed with narcolepsy a shorter time after debut of symptoms due to the awareness of narcolepsy as a possible side effect of the vaccine. Moreover, the mean (SD) disease duration was 5.4 years (1.5) in our cohort, thus the range in disease duration was somewhat limited.

In Paper III, we found that the presence of medical comorbidity in children was positively associated with BMI. There is an increased prevalence of medical comorbidity in obese patients without narcolepsy, and the finding is therefore not unexpected (156). However, it highlights the importance of carefully monitoring patients with narcolepsy in regard to overweight and obesity. Obesity is a major risk factor for developing cardiovascular disorders, and there is increasing evidence that childhood obesity is significantly associated with cardiovascular risk-factors, such as high blood pressure, in adulthood (156).

\subsection{Fatigue}

Our finding of a high prevalence of fatigue is in accordance with previous studies on SN patients $(14,114)$. Further, the fatigue scores show more severe fatigue compared to other medical conditions such as rheumatoid arthritis (136) and cancer (157).

There is a positive relation between disturbed nocturnal sleep and fatigue in healthy subjects (158). However, the direction of the association between nocturnal sleep disturbances and daytime fatigue remains unclear (113). Certain psychiatric disorders are also associated with fatigue (159), and Lecendreux et al. found that fatigue, in addition to EDS and insomnia, was associated with ADHD symptoms regardless of treatment in patients with narcolepsy, and questioned if ADHD symptoms could be secondary to fatigue (94).

Droogleever et al. found that severe fatigue was associated with a significantly increased functional impairment, increased depressive symptoms and a lowered general quality of life in patients with narcolepsy (14). The authors discussed whether fatigue was just an equivalent of depression in the narcolepsy population. They argue that severe fatigue was 
not explained exclusively by the presence of major depression in narcolepsy alone since use of antidepressant was not associated with fatigue severity.

\subsection{Hypocretin}

We found no significant associations between psychiatric symptoms and hypocretin 1 levels in CSF (Paper II), which is in agreement with the study of Lecendreux et al. (94). Interestingly, Brundin and coworkers found a significant correlation between CSF hypocretin levels and specific psychiatric symptoms in suicide attempters and suggested that reduced hypocretin levels are involved in the etiology of specific psychiatric symptoms (160). The role of hypocretin in the pathogenesis of psychiatric symptoms in patients with narcolepsy is not fully understood. However, animal studies show that hypocretinergic neurons interact with several areas of the brain involved in stress reactions, reward, cognition and emotion (161163).

We found no significant association between obesity and hypocretin 1 levels in CSF (Paper III). Central administration of hypocretin increases food intake (62), therefore, decreased hypocretin would be expected to reduce food consumption and decrease body weight, and not the opposite. Nishino et al. reported in their study that the mean BMI of narcolepsy patients with undetectable CSF hypocretin was significantly higher than that of patients who had detectable CSF hypocretin levels and suggest that this favors more direct effects of hypocretin deficiency on energy metabolism (73). Inocente et al. found in their study on children with NT1, that obese children had lower CSF hypocretin 1 levels than the non-obese children. However, only few CSF samples were available, and obesity was also prevalent in the children without cataplexy, in whom the hypocretin 1 levels were expected to be normal. The authors therefore suggested that factors other than hypocretin 1 levels were involved (12).

Since hypocretin also regulates sympathetic activity and the neuroendocrine system, decreased levels of hypocretin may reduce the metabolic rate through this system. In favor to this theory, Poli et al. found an association between narcolepsy and BMI-independent metabolic alterations. BMI was greater in NT1 patients compared to patients with IH despite 
significantly decreased food intake in NT1 patients, implying that there is a dysmetabolism that is not caused by altered food consumption (106).

\subsection{Post Pandemrix narcolepsy and sporadic narcolepsy}

Initially, it was speculated if early debut of narcolepsy symptoms, abrupt onset, severe cataplexy and severe psychiatric comorbidity seen in some patients vaccinated with Pandemrix represented a different narcolepsy phenotype or a more full blown version (5, 47). However, studies comparing PPN with SN patients have shown only minor differences $(47,164)$. Pizza et al. found a more disrupted sleep pattern with more frequent awakenings and shorter mean MSLT in PPN patients compared to SN patients (164). Dauvilliers et al. found a higher number of sleep onset REM periods in addition to a shorter diagnostic delay in vaccinated patients (47). The majority of the participants in our project were H1N1 vaccinated with Pandemrix and no direct comparison within the cohort could therefore be made. Nevertheless, we can compare our findings with previous studies on SN patients.

In Paper I, we saw impairment in HRQoL as previous studies on patients with PPN and SN $(82,85,95,141)$. The improvement in HRQoL seen in our study is in contrast to the follow-up study of Sarkanen et al. on patients with PPN who found no significant improvement after 2 years of follow-up and to the study of Vignatelli et al., measuring HRQoL in SN patients after 5 years of follow-up.

In Paper II, we found a high prevalence of psychiatric symptoms, which also has been found in several studies on SN as well as PPN. Partinen et al. suggested that psychiatric symptoms were perhaps more severe in PPN compared to SN since several of the children in his study needed long term psychiatric hospitalization and even antipsychotic treatment (5). Szakacs et al. found that psychiatric symptoms seemed to be more frequent in PPN patients, but as there were 31 PPN and only 7 SN patients comparison between the groups was difficult (95).

Our findings in Paper III, showing overweight /obesity in 54\% of the children and $61 \%$ of the adults is in line with previous prevalence reports on $\mathrm{BMI}$ in patients with $\mathrm{SN}(12,13,101)$. Sarkanen et al. found in their follow-up study an increase in BMI in patients with PPN and $\mathrm{SN}$, but the majority of their subjects remained within the normal limit. They also found a 
greater BMI in the SN group than in the PN group which they suspected was due to a greater age and longer disease durations in the SN group (165). The mean disease duration in our study was longer than in the study of Sarkanen, differing approximately 2 years. Pizza et al. compared patients with SN and PNN and found no significant difference in BMI between the groups, only a non-significant trend towards more overweight and obesity in SN vs PPN patients (164). 


\section{METHODOLOGICAL CONSIDERATIONS}

In this clinical thesis we have measured HRQoL, the extent of psychiatric symptoms and the level of medical comorbidities in order to respond to the request from The Norwegian Ministry of Health and Care Services to survey the clinical phenotype of the narcolepsy that abruptly increased in incidence following the H1N1 influenza epidemic and subsequent mass vaccination with Pandemrix in 2009. In the following section we address the strengths and weaknesses of the study designs, methodological problems regarding study samples, study participants, the use of questionnaires and statistics. Detailed descriptions of the methods are presented in the three papers.

\subsection{Study design}

This thesis is comprised of three studies, two cross-sectional and one prospective. General strengths and limitations regarding design are provided in Table 3. There are some general considerations that should be addressed. The presence of selection bias is possible if those who declined to participate differ significantly from those who accepted our invitation. Selection bias results in problems with generalizability (the extent to which the observed results represent the truth in the whole study-population). An example of selection bias potentially present in our study is differential nonresponse between those with well controlled or less severe narcolepsy and those with extremely severe and disabling narcolepsy symptoms can exist.

Paper I is a prospective cohort study that allowed us to investigate how some predefined predictors over time influenced the outcome, HRQoL in our statistical model. We had considerable difficulties as many participants chose not to return the baseline questionnaire. When such attrition occurs, and data are missing at random, the power of the study is weakened, and type II errors (non-rejection of a false null hypothesis) may occur. This might be a challenge in heath studies. If the characteristics of those who do not respond are different from those who respond, the results of the responders are not representative for the broader group of people that have the disease. This can negatively impact internal validity (extent results are free from unmeasured confounding), but primarily poses challenges for external validity (extent to which the results of a study can be generalized). To 
examine selection bias in Paper I, we compared core narcolepsy symptoms, age, and gender from the participants in the study with the remaining cohort (84 patients at the time) using Fisher exact tests. No significant differences were observed, but we cannot exclude the presence of some selection bias.

In paper II and III a cross-sectional design was applied. In general, a cross sectional study design is particularly suitable for estimating the prevalence of a behavior or a disease in a population and serves the study purposes well (166). All data are collected at a single point in time although recruitment may take place across a longer period of time. There is therefore no temporal ordering and so it is difficult to infer cause and effect (166). Therefore, we can only conclude that our results indicate an association between exposure and effect that may be clinically relevant.

Table 3. General strengths and limitations of the applied study designs

\begin{tabular}{|lllll|}
\hline Paper & Design & Limitation & Strength \\
\hline I & Prospective cohort study & - Maintaining follow-up may be & - Knowledge of \\
& difficult & sequence of events \\
& -Exposure may be linked to a hidden & (temporality) \\
& confounder & - Examines multiple \\
& - For rare diseases, large sample size & outcomes for a given \\
& or long follow up is necessary & exposure \\
\hline II \& III & Cross-sectional study & -Cannot be used to infer causality & - Descriptive study well \\
& (exposures and outcomes are & adapted to assess the \\
& measured simultaneously) & frequency and \\
& & distribution of a \\
& & particular disease in a \\
& & defined population \\
& &
\end{tabular}




\subsection{Study sample}

The study sample was homogenous in that the vast majority of the participants in the three papers were of Norwegian ethnicity, all had debut of symptoms after 2009, and the disease duration was consequently short. However, the sample showed some heterogeneity in that it included young children, adolescents and adults from different social classes and from various parts of Norway. The level of medical comorbidities and the extent of psychiatric symptoms may, due to natural variability, be varied and significantly different between subgroups. Accordingly, children and adults were analyzed separately (Paper II and III). This decreases the power to detect possible associations between various exposures and outcomes and increases the risk of type II errors (non-rejection of a false null hypothesis).

Our studies show that NT1 is heterogeneous disorder in regard to severity of symptoms and degree of comorbidities. All patients in our study had debut of symptoms after 2009 and accordingly the disease duration was relatively short. However, we observed fairly significant variation considering the range of possible values in this variable. Nevertheless, it is possible that a greater sample size might have influenced our result.

It is likely that socioeconomic differences may play a significant part in the diagnosis, treatment and ability to adapt to the symptoms of narcolepsy. Unfortunately, the impact of socioeconomic status was not addressed in any of the studies in this thesis and may confound our results.

The majority of the patients in the studies in this thesis were Pandemrix vaccinated. (29/31 in Paper I, 70/76 in Paper II and 80/91 in Paper III). Separate linear regression analysis showed no significant differences in the mean total psychiatric symptoms scores (Paper II) and mean BMI (Paper III) between the vaccinated and unvaccinated patient groups, so the two groups were subsequently analyzed together. We cannot disprove that small undetected differences between the groups may exist, but find it unlikely that this pooling has substantially affected the model parameter estimations.

\subsection{Control group}

A clear limitation in all three studies was the lack of a control group. In order to at least partly overcome this problem our results were compared to national reference data (Paper 
I), and we used well-validated and reliable measures, allowing for generalizable model estimation. Classification of overweight and obesity (Paper III) was based on percentiles from the Bergen study based on IOTF's criteria (167), and is validated in the Norwegian population. Regardless of our efforts to reduce potential bias, the presence of a control group would have increased the validity and precision of our results significantly.

\subsection{Sample sizes}

NT1 is a rare disorder, and it is therefore challenging to obtain a large sample size. The study population in Paper II and III in relation to the rarity of the disease is relatively large, but we acknowledge the presence of lack of power and subsequently the potential to conduct type II error, particularly in paper I.

\subsection{Study procedure}

As recommended in the ICSD-3 criteria for diagnosing circadian rhythm disorders, we aimed to perform actigraphy recordings 14 days prior to PSG/MSLT investigations, although a total of 14 days recordings was not always possible. The AASM guidelines for performing MSLT recordings recommends to terminate the MSLT recording after 20 minutes if sleep does not occur, or after 15 minutes of sleep (168). However, for practical reasons, all subjects were given the opportunity to sleep for 30 minutes regardless of whether sleep occurred or not. This may have decreased the sleep pressure for the following nap.

All interpretations of the sleep investigations were performed by the same sleep technicians (Rannveig Viste and Janita Vevelstad), under the supervision of a neurophysiologist (Kristian Bernhard Nilsen) and a neurologist and sleep specialist (Stine Knudsen Heier). All handling with HRQoL and psychiatric symptoms questionnaires were performed by the candidate, under the supervision of a child psychiatrist (Berit Hjelde Hansen). This allows for accuracy in the parameters used in the analyses and strengthens our studies.

To assess extent of medical comorbidity in Paper III, we used a predefined comprehensive list of disorders. We also asked patients and/or parents if the patient have or have had other disorders than what was not on the list. Interviews were performed by medical doctors (Hilde Therese Juvodden, Stine Knudsen Heier and the candidate), and symptoms and 
diagnoses were thoroughly evaluated. The self-reported diagnoses were not confirmed by previous medical records. We chose to omit comorbid sleep disorders from the analyzes in Paper III, because several of the patients were unware of having an additional sleep disorder prior to inclusion and this was diagnosed after defining symptoms through the interviews and through sleep investigations. Other sleep disorders are present in patients with NT1, and analyzing the prevalence of comorbid sleep disorders would have been interesting. Furthermore, additional sleep disorders may have confounded our results.

\subsection{Questionnaires}

A weakness in the questionnaires used in Papers I, II and III is that there may be individual variations in the answers over time, and that other aspects of life may influence the answers. There is also a probability of recall bias.

\section{Stanford Sleep Questionnaire}

Although there is a pediatric version of the EES, ESS-CHAD (169), we used the same translated original version of EES as a measure of excessive daytime sleepiness in children and adults in Paper I and in a sub analyzes in Paper II and Paper III. The EES and the ESSCHAD is similar with the exception of minor differences in the description of some of the activities with the purpose of making the questions more comprehensible. Although all participants in our cohort were requested to answer the questions as if they had been in the given or equivalent situation, if the given situation had not actually taken place, we acknowledge the lack of accuracy as a limitation. To assess narcolepsy symptoms we used SSQ which is validated in several populations (170) although not yet in Norway. We used the frequency of cataplexy as a measure of cataplexy severity in Paper III. It can be questioned if this is a reliable measure for cataplexy severity. Others have used the degree of involvement of different muscle groups in a cataplexy attack as a cataplexy severity measurement (12, 171). 


\section{$\underline{\text { Health related quality of life }}$}

PedsQL (generic core scale) is a non-disease specific survey. It has been proposed as a valid and reliable generic pediatric HRQOL measurement in the age group ranging from 2 to 18 years (134), and can also be used in clinical practice, clinical trials and research (172). The Norwegian PedsQL has shown validity and reliability for the age groups 13-15 years (172). PedsQL scale has previously been used in studies of patients with narcolepsy (141). The advantage of non-disease specific surveys in contrast to a disease specific survey is that it permits comparison across different disorders and populations.

There are, however, items in PedsQL that may be interpreted as disease specific by NT1 patients, such as items related to difficulties in conducting sports or exercise, sleeping problems, or concentration difficulties. These problems may be consequences of narcolepsy symptoms, and as the survey is not disease specific, the results may be difficult to interpret and less comparable to other populations. Disease-specific questionnaires are more likely to detect disease specific symptoms and are more sensitive to changes over time (173). To our knowledge the only validated disease specific HRQoL questionnaire for narcolepsy, is the NARQoL-21, which was published in 2019 (83). Changes in clinical status are perhaps more easily seen when using disease-specific questionnaires as they are more sensitive and clinically relevant (173).

\section{Psychiatric symptoms}

The benefits of using a validated standardized questionnaire like the ASEBA, are that the answers can be interpreted with precision on a group level. Further, that the patient's perspective is highlighted. It does not necessarily imply that their perceptions are in agreement with a clinical psychiatric disorder. The use of additional diagnostic tools could have strengthened our findings.

The ASEBA CBCL is a widely used instrument for assessing child and adolescent and emotional problems in several situations, such as for screening purposes and in research (174). It has been used in two previous studies on patients with narcolepsy $(85,141)$. A study in a Norwegian population supports a predictive validity of the CBCL to discriminate between 
children with and without a psychiatric disorder (174). Although the ASEBA ASR is also a widely used tool, it is to our knowledge not yet validated in a Norwegian population.

In Papers I and II, psychiatric symptoms were rated by parents for those aged below 18 years while we used a self-reported version for those aged above 18 years. Discrepancies in clinical assessment of psychiatric symptoms measured by ASEBA between multiple informants have been described $(175,176)$. In non-clinical study samples, youths tend to report a higher severity of symptoms than their parents $(176,177)$, but in a clinically-referred study population, parents are more likely to report a higher severity of symptoms (176). A metaanalysis showed greater correlation between the ratings for younger than for older children (178). We acknowledge that the use of both self-report and parent-report, depending on the age, could potentially be a limitation. However, due to the age span in our cohort, the use of either a self-reported version or a parent reported version only would have produced bias as well.

\subsection{Statistical perspectives}

\subsubsection{General considerations}

In order to secure adequate statistical methods we have collaborated with statistician David Swanson, PhD, Oslo Centre for Biostatistics and Epidemiology, OUH, Oslo. Detailed descriptions of the statistical methods used are listed in the original papers. There are some general considerations which should be discussed. Sample size is important when conducting statistical analysis. Due to logistical challenges and the time limit of this project, we were unable to increase our sample size. A large proportion of the cohort preferred to be included during the holidays in order to minimize absence from school and work. Moreover, patients were included simultaneously as their first-degree relatives who participated in the larger national study-project (Phenotype and pathogenesis of narcolepsy following H1N1vaccination in Norway), diminishing the time available for inclusion for the patients. Furthermore, all participants received multidisciplinary follow-up and the best available treatment. Therefore, randomization to a specific treatment in a trial was not considered. 


\subsubsection{Variability in chosen parameters}

The chosen explanatory variables in all the studies are considered as the core NT1 symptoms and factors that are most likely to impact the outcome. In some of these variables used, such as MSLT values, hypocretin 1 values in CSF and disease duration, the variability is limited. However, while the distribution for hypocretin and MSLT are zero-inflated and right-skewed, respectively, their ranges are adequate and do not deviate from what one would expect for these measures in studies focusing on narcolepsy. While a more uniform distribution would be slightly more well-powered, it would be uncommon for these variables to be distributed in this way for a representative sample from our population. Disease duration (explanatory variable, Paper III) exhibited fairly significant variation considering the range of possible values. Note that regression model test statistics are invariant under rescaling of the variables, so differences in variability in any absolute sense have no influence on power or calculation of test statistics.

\subsubsection{Paper I}

In Paper I, the primary aim was to describe changes in HRQoL after two years of multidisciplinary follow-up. The secondary aim was to explore possible predictors to the changes in HRQoL, using subjective rated EDS, the use of sodium oxybate at follow-up and psychiatric symptoms as independent variables in a prospective design.

We used paired samples t-test to measure the change in HRQoL from baseline to follow-up. The paired samples t-test is commonly used in studies with repeated measures design and has four main assumptions that were met by the repeated measurement of HRQoL: 1) The dependent variable was continuous. 2) The observations within each sample are independent of one another. 3) The dependent variable was normally distributed. 4) The dependent variable did not contain outliers (166).

Furthermore, we divided the study participants into two groups based on their baseline total HRQoL summary score (greater or less than the baseline mean) and compared the change between baseline and follow-up in the two groups using independent samples t-test. However, this analysis may be biased by the phenomenon "regression to the mean", which is the tendency for more extreme observations to return to their mean value on subsequent 
follow-up or observation. It occurs because those extreme observations result from chance realizations of a larger error term, not sampling from a distribution with a genuinely larger mean.

\subsubsection{Paper II}

In Paper II, the study objective was to explore the extent of psychiatric symptoms in a cohort of Norwegian NT1 patients and to explore possible causes of the psychiatric symptoms seen in NT1. The cross-sectional design is suitable for exploring the extent of psychiatric symptoms, but insufficient to show causes of psychiatric symptoms. Therefore we used "possible causes" since a causal relationship cannot be established with this design.

Regression analysis allowed us to isolate the role of each independent variable by estimating the effect that changing one independent variable has on the dependent variable while holding all the other independent variables constant. We included independent variables that in our opinion were likely to have an effect on the dependent variable with the purpose of reducing confounding bias. We checked residual plots and observed that our model fit the data adequately.

\subsubsection{Paper III}

The primary aim in Paper III was to study the extent of medical comorbidities, in particular being overweight and obese. Further goals included exploring the extent of fatigue and possible associations between being overweight/obese and these factors: age at disease onset, disease duration, various indicators of severity of narcolepsy (cataplexy, sleepiness, fragmented sleep, hypocretin 1 levels), and additional comorbidities (psychiatric symptoms, medical comorbidities, fatigue).

The cross-sectional design is well suited for the primary aim of the study. Linear regression analyses were used to assess possible associations between independent and dependent variables. The benefits and possible limitations of linear regression which are described 
above are also applicable here. We also used the same principles for selection of variables in our model and checking of residual plots. 


\section{CONCLUSIONS AND CLINICAL RELEVANCE}

In this thesis we characterized the clinical phenotype of patients who developed NT1 after the H1N1 epidemic and vaccination campaign in 2009. We believe that this project may lead to a better understanding of the disorder as we throw light on clinical relevant issues. The findings in this thesis show that NT1 phenotype in patients that developed NT1 after the H1N1 epidemic and vaccination camping in Norway is complex and associated with several comorbidities and impaired HRQoL.

In Paper I, we found impaired HRQoL, but we saw an improvement in HRQoL after 2 years of follow-up. The only independent variable associated with improvement in HRQoL was the use of sodium oxybate at follow-up. Baseline value of subjective rated EDS and psychiatric symptoms had no impact on the changes in HRQoL, implicating that regardless of the severity of these, improvement is attainable.

In Paper II, it was confirmed that NT1 is complicated with a high prevalence of psychiatric symptoms, as seen in previous studies on PPN and SN. Internalizing problems were the major concern in both children and adults. Further, we investigated plausible explanations to psychiatric symptoms that may have clinical importance. We found no associations between psychiatric symptoms and EDS or hypocretin 1 levels in CSF, but to our surprise, we found that fragmented sleep was negatively associated with psychiatric symptoms in children but positively associated with psychiatric symptoms in adults. Only speculations can be made as to whether the latter finding may be due to the methods used in the study, or if there are other plausible explanations.

In paper III, we showed that approximately half of the subjects in our cohort reported to have one or several medical comorbidities. This is not markedly increased compared to the general Norwegian population and in line with previous studies on patients with NT1. In agreement with other studies on PPN and SN patients, we observed a high prevalence of overweight/obesity, also when compared to data provided by the national studies. BMI was not associated with age at disease onset, disease duration or the severity of the narcolepsy phenotype measured by severity of symptoms or additional comorbidities. We did, however, find that subjectively rated EDS was associated with BMI in children when this was tested in a separate analyses. Further, the presence of medical comorbidity was associated with BMI 
in children. We conclude that subjects with narcolepsy are at greater risk of being overweight and obese.

Initial reports speculated that the phenotype of PPN was different than that of SN. The studies included in this thesis, does, however, not support a marked difference between the phenotype of patients that developed NT1 after the H1N1 vaccination campaign and previous SN patients. However, the narcolepsy phenotype may vary according to age, as illustrated in our papers assessing psychiatric symptoms and obesity. Narcolepsy is a life-long disorder with a major impact on quality of life. Symptoms often first appear in childhood, and early recognition may be important.

Our studies highlight the value of monitoring patients with narcolepsy and to pursue individual and close follow-up in order to prevent comorbidity and secondary complications and to enable proper treatment and improve HRQoL. 


\section{FUTURE PERSPECTIVES}

It is poorly understood why there are such variations in the NT1 phenotype and why NT1 is accompanied by several comorbidities. Longitudinal studies focusing on these aspects and national and/or international quality-register studies could be of great importance.

Furthermore, variations in psychiatric symptoms over time may influence $H R Q$ oL, and interventional studies on psychiatric symptoms and comorbidities would be clinically relevant.

Obesity is a potent risk factor for both medical and psychiatric disorders as well as impaired HRQoL. Individuals with narcolepsy would probably benefit from comprehensive intensive lifestyle intervention programs focusing on diet and physical activity. This should be addressed in future interventional studies. In our opinion this is perhaps the most important topic from a patient perspective. 


\section{ETHICAL CONSIDERATIONS}

\subsection{Ethical approval and practice}

All protocols have been approved by the Regional Committees for Medical and Health Research Ethics (REK) (REK number 2014/450). The researched has followed the ethical principles in agreement with the Declaration of Helsinki, developed by the World Medical Association. Written informed consent was signed by the participants before inclusion in the studies of this thesis and for participants younger than 16 years of age, participants was well informed and consent was signed by their parents.

\subsection{Ethical perspectives}

After the H1N1 influenza vaccination campaign in 2009, NevSom was delegated by the Norwegian Ministry of Health and Care Services, the task to survey individuals that had developed NT1 after 2009. The current PhD project is a part of the comprehensive work carried out by NevSom. Other PhD projects are also involved in this work. If participants did not want to take part in the whole project, they could still choose to be included in parts of the project. They could at any time withdraw consent.

The period of inclusion for each patient and their family lasted for 2-4 days. During this time, participants received a thorough evaluation of symptoms, social functioning, comorbidities and previous medication by a multidisciplinary team. They were educated about narcolepsy, given school and work advice, and if necessary, medical and non-medical treatment advice. These benefits were not given to patients and relatives that declined participation, or that were unable to participate in the projects for other reasons. 


\section{REFERENCES}

1. American Academy of Sleep Medicine. (AASM) Internation Classification of Sleep Disorders 3ed: Darien, IL: American Academy of Sleep Medicine; 2014.

2. Kornum BR, Knudsen S, Ollila HM, Pizza F, Jennum PJ, Dauvilliers Y, et al. Narcolepsy. Nature Reviews Disease Primers. 2017;3:16100.

3. Heier MS, Gautvik KM, Wannag E, Bronder KH, Midtlyng E, Kamaleri Y, et al. Incidence of narcolepsy in Norwegian children and adolescents after vaccination against H1N1 influenza A. Sleep Med. 2013;14(9):867-71.

4. Szakacs A, Darin N, Hallbook T. Increased childhood incidence of narcolepsy in western Sweden after H1N1 influenza vaccination. Neurology. 2013;80(14):1315-21.

5. Partinen M, Saarenpaa-Heikkila O, Ilveskoski I, Hublin C, Linna M, Olsen P, et al. Increased incidence and clinical picture of childhood narcolepsy following the $2009 \mathrm{H} 1 \mathrm{~N} 1$ pandemic vaccination campaign in Finland. PLoS One. 2012;7(3):e33723.

6. Dauvilliers Y, Montplaisir J, Cochen V, Desautels A, Einen M, Lin L, et al. Post-H1N1 narcolepsy-cataplexy. Sleep. 2010;33(11):1428-30.

7. Postiglione E, Antelmi E, Pizza F, Lecendreux M, Dauvilliers Y, Plazzi G. The clinical spectrum of childhood narcolepsy. Sleep Med Rev. 2017.

8. Ohayon MM. Narcolepsy is complicated by high medical and psychiatric comorbidities: a comparison with the general population. Sleep Med. 2013;14(6):488-92.

9. Fortuyn HA, Lappenschaar MA, Furer JW, Hodiamont PP, Rijnders CA, Renier WO, et al. Anxiety and mood disorders in narcolepsy: a case-control study. Gen Hosp Psychiatry. 2010;32(1):4956.

10. Cohen A, Mandrekar J, St Louis EK, Silber MH, Kotagal S. Comorbidities in a community sample of narcolepsy. Sleep Med. 2018;43:14-8.

11. Poli F, Pizza F, Mignot E, Ferri R, Pagotto U, Taheri S, et al. High prevalence of precocious puberty and obesity in childhood narcolepsy with cataplexy. Sleep. 2013;36(2):175-81.

12. Inocente CO, Lavault S, Lecendreux M, Dauvilliers Y, Reimao R, Gustin MP, et al. Impact of obesity in children with narcolepsy. CNS Neurosci Ther. 2013;19(7):521-8.

13. Schuld A, Hebebrand J, Geller F, Pollmacher T. Increased body-mass index in patients with narcolepsy. Lancet. 2000;355(9211):1274-5.

14. Droogleever Fortuyn HA, Fronczek R, Smitshoek M, Overeem S, Lappenschaar M, Kalkman J, et al. Severe fatigue in narcolepsy with cataplexy. J Sleep Res. 2012;21(2):163-9.

15. Daniels E, King MA, Smith IE, Shneerson JM. Health-related quality of life in narcolepsy. J Sleep Res. 2001;10(1):75-81.

16. Dodel R, Peter H, Spottke A, Noelker C, Althaus A, Siebert U, et al. Health-related quality of life in patients with narcolepsy. Sleep Med. 2007;8(7-8):733-41.

17. Đogas Z, Pecotic R, Valic M. Physiological basis of sleep. Regulation of sleep and wakefulness. ESRS sleep medicine textbook. Regensburg: European Sleep Research Society; 2014. p. 13-25.

18. Siegel JM. Why we sleep. Sci Am. 2003;289(5):92-7.

19. Sateia MJ. Update on sleep and psychiatric disorders. Chest. 2009;135(5):1370-9.

20. Berry RB, Harding SM. Sleep and medical disorders. J Medical Clinics. 2004;88(3):679-703.

21. Moore RY. Suprachiasmatic nucleus in sleep-wake regulation. J Sleep medicine. 2007;8:27-

33.

22. Westphal C. Eigetumliche mit Einschlafen verbundene Anfalle. Arch Psychiatr Nervenkr.

1877;7:631-5.

23. Mignot E. A hundred years of narcolepsy research. Arch Ital Biol. 2001;139(3):207-20.

24. Gélineau J. De la narcolepsie. Gazette des hôpitaux. 1880;53:626-8.

25. Löwenfeld L. Ueber narkolepsie. Munch Med Wochenschr. 1902;49:1041-5. 
26. Economo C. Encephalitis lethargica: its sequelae and treatment: Oxford University Press; 1931.

27. American Academy of Sleep Medicine. (AASM) Internation Classification of Sleep Disorders; diagnostic and coding manual. 2 ed: Westchester, IL: American Academy of Sleep Medicine; 2005.

28. Mignot E, Lin L, Rogers W, Honda Y, Qiu X, Lin X, et al. Complex HLA-DR and -DQ interactions confer risk of narcolepsy-cataplexy in three ethnic groups. Am J Hum Genet. 2001;68(3):686-99.

29. Peyron C, Tighe DK, van den Pol AN, de Lecea L, Heller HC, Sutcliffe JG, et al. Neurons containing hypocretin (orexin) project to multiple neuronal systems. J Neurosci. 1998;18(23):999610015.

30. Dauvilliers Y, Arnulf I, Mignot E. Narcolepsy with cataplexy. Lancet. 2007;369(9560):499-511.

31. Challamel M-J, Mazzola M-E, Nevsimalova S, Cannard C, Louis J, Revol M. Narcolepsy in children. Sleep. 1994;17(suppl_8):S17-S20.

32. Marcus $\mathrm{CL}$, Trescher $\mathrm{WH}$, Halbower AC, Lutz J. Secondary narcolepsy in children with brain tumors. Sleep. 2002;25(4):427-31.

33. Malik S, Boeve BF, Krahn LE, Silber MH. Narcolepsy associated with other central nervous system disorders. Neurology. 2001;57(3):539-41.

34. Lankford DA, Wellman JJ, O'Hara C. Posttraumatic narcolepsy in mild to moderate closed head injury. Sleep. 1994;17(suppl_8):S25-S8.

35. Overeem S, Dalmau J, Bataller L, Nishino S, Mignot E, Verschuuren J, et al. Hypocretin-1 CSF levels in anti-Ma2 associated encephalitis. Neurology. 2004;62(1):138-40.

36. Overeem S, Mignot E, van Dijk JG, Lammers GJ. Narcolepsy: clinical features, new pathophysiologic insights, and future perspectives. J Clin Neurophysiol. 2001;18(2):78-105.

37. Nevsimalova S. Narcolepsy in childhood. Sleep Med Rev. 2009;13(2):169-80.

38. Wise MS, Lynch J. Narcolepsy in children. Semin Pediatr Neurol. 2001;8(4):198-206.

39. Ohayon MM, Priest RG, Zulley J, Smirne S, Paiva T. Prevalence of narcolepsy symptomatology and diagnosis in the European general population. Neurology. 2002;58(12):1826-33.

40. Lewin DS, Rosen RC, England SJ, DahI RE. Preliminary evidence of behavioral and cognitive sequelae of obstructive sleep apnea in children. Sleep Med. 2002;3(1):5-13.

41. Liu X, Liu L, Owens JA, Kaplan DL. Sleep patterns and sleep problems among schoolchildren in the United States and China. Pediatrics. 2005;115(1 Suppl):241-9.

42. Allen RP, Earley CJ. Restless legs syndrome: a review of clinical and pathophysiologic features. J Clin Neurophysiol. 2001;18(2):128-47.

43. Ivanenko A, Crabtree VM, Obrien LM, Gozal D. Sleep complaints and psychiatric symptoms in children evaluated at a pediatric mental health clinic. J Clin Sleep Med. 2006;2(1):42-8.

44. Longstreth WT, Jr., Koepsell TD, Ton TG, Hendrickson AF, van Belle G. The epidemiology of narcolepsy. Sleep. 2007;30(1):13-26.

45. Wijnans L, Lecomte C, de Vries C, Weibel D, Sammon C, Hviid A, et al. The incidence of narcolepsy in Europe: before, during, and after the influenza $A(H 1 N 1) p d m 09$ pandemic and vaccination campaigns. Vaccine. 2013;31(8):1246-54.

46. Silber MH, Krahn LE, Olson EJ, Pankratz VS. The epidemiology of narcolepsy in Olmsted County, Minnesota: a population-based study. Sleep. 2002;25(2):197-202.

47. Dauvilliers Y, Arnulf I, Lecendreux M, Monaca Charley C, Franco P, Drouot X, et al. Increased risk of narcolepsy in children and adults after pandemic H1N1 vaccination in France. Brain. 2013;136(Pt 8):2486-96.

48. Nohynek H, Jokinen J, Partinen M, Vaarala O, Kirjavainen T, Sundman J, et al. AS03 adjuvanted AH1N1 vaccine associated with an abrupt increase in the incidence of childhood narcolepsy in Finland. PLoS One. 2012;7(3):e33536.

49. Dauvilliers Y, Montplaisir J, Molinari N, Carlander B, Ondze B, Besset A, et al. Age at onset of narcolepsy in two large populations of patients in France and Quebec. Neurology. 2001;57(11):202933.

50. Morrish E, King MA, Smith IE, Shneerson JM. Factors associated with a delay in the diagnosis of narcolepsy. Sleep Med. 2004;5(1):37-41. 
51. Han F, Lin L, Warby SC, Faraco J, Li J, Dong SX, et al. Narcolepsy onset is seasonal and increased following the 2009 H1N1 pandemic in China. Ann Neurol. 2011;70(3):410-7.

52. Ohayon MM, Okun ML. Occurrence of sleep disorders in the families of narcoleptic patients. Neurology. 2006;67(4):703-5.

53. Honda Y. Discrimination of narcoleptic patients by using genetic markers and HLA. Sleep Research. 1983;12:254-8.

54. Lande A, Andersen I, Egeland T, Lie BA, Viken MK. HLA -A, -C, -B, -DRB1, -DQB1 and -DPB1 allele and haplotype frequencies in 4514 healthy Norwegians. Hum Immunol. 2018;79(7):527-9. 55. Parkes JD, Langdon N, Lock C. Narcolepsy and immunity. Br Med J (Clin Res Ed). 1986;292(6517):359-60.

56. Fontana A, Gast H, Reith W, Recher M, Birchler T, Bassetti CL. Narcolepsy: autoimmunity, effector T cell activation due to infection, or T cell independent, major histocompatibility complex class II induced neuronal loss? Brain. 2010;133(Pt 5):1300-11.

57. Singh AK, Mahlios J, Mignot E. Genetic association, seasonal infections and autoimmune basis of narcolepsy. J Autoimmun. 2013;43:26-31.

58. Aran A, Einen M, Lin L, Plazzi G, Nishino S, Mignot E. Clinical and therapeutic aspects of childhood narcolepsy-cataplexy: a retrospective study of 51 children. Sleep. 2010;33(11):1457-64.

59. Cvetkovic-Lopes V, Bayer L, Dorsaz S, Maret S, Pradervand S, Dauvilliers Y, et al. Elevated Tribbles homolog 2-specific antibody levels in narcolepsy patients. The Journal of clinical investigation. 2010;120(3):713-9.

60. Lind A, Ramelius A, Olsson T, Arnheim-Dahlström L, Lamb F, Khademi M, et al. A/H1N1 antibodies and TRIB2 autoantibodies in narcolepsy patients diagnosed in conjunction with the Pandemrix vaccination campaign in Sweden 2009-2010. J Autoimmun. 2014;50:99-106.

61. De Lecea L, Kilduff T, Peyron C, Gao X-B, Foye P, Danielson P, et al. The hypocretins: hypothalamus-specific peptides with neuroexcitatory activity. Proc Natl Acad Sci U S A. 1998;95(1):322-7.

62. Sakurai T, Amemiya A, Ishii M, Matsuzaki I, Chemelli RM, Tanaka H, et al. Orexins and orexin receptors: a family of hypothalamic neuropeptides and $\mathrm{G}$ protein-coupled receptors that regulate feeding behavior. Cell. 1998;92(4):573-85.

63. Taheri S, Zeitzer JM, Mignot E. The role of hypocretins (orexins) in sleep regulation and narcolepsy. Annu Rev Neurosci. 2002;25(1):283-313.

64. Lin L, Faraco J, Li R, Kadotani H, Rogers W, Lin X, et al. The sleep disorder canine narcolepsy is caused by a mutation in the hypocretin (orexin) receptor 2 gene. Cell. 1999;98(3):365-76.

65. Nishino S, Ripley B, Overeem S, Lammers GJ, Mignot E. Hypocretin (orexin) deficiency in human narcolepsy. Lancet. 2000;355(9197):39-40.

66. Thannickal TC, Moore RY, Nienhuis R, Ramanathan L, Gulyani S, Aldrich M, et al. Reduced number of hypocretin neurons in human narcolepsy. Neuron. 2000;27(3):469-74.

67. Nevárez N, de Lecea LJF. Recent advances in understanding the roles of hypocretin/orexin in arousal, affect, and motivation. 2018;7.

68. Sakurai T. The neural circuit of orexin (hypocretin): maintaining sleep and wakefulness.

Nature Reviews Neuroscience. 2007;8(3):171.

69. Sakamoto F, Yamada S, Ueta Y. Centrally administered orexin-A activates corticotropinreleasing factor-containing neurons in the hypothalamic paraventricular nucleus and central amygdaloid nucleus of rats: possible involvement of central orexins on stress-activated central CRF neurons. Regul Pept. 2004;118(3):183-91.

70. Giardino WJ, de Lecea L. Hypocretin (orexin) neuromodulation of stress and reward pathways. Curr Opin Neurobiol. 2014;29:103-8.

71. Yamanaka A, Kunii K, Nambu T, Tsujino N, Sakai A, Matsuzaki I, et al. Orexin-induced food intake involves neuropeptide Y pathway. Brain Res. 2000;859(2):404-9.

72. Hara J, Beuckmann CT, Nambu T, Willie JT, Chemelli RM, Sinton CM, et al. Genetic ablation of orexin neurons in mice results in narcolepsy, hypophagia, and obesity. Neuron. 2001;30(2):345-54. 
73. Nishino S, Ripley B, Overeem S, Nevsimalova S, Lammers GJ, Vankova J, et al. Low cerebrospinal fluid hypocretin (Orexin) and altered energy homeostasis in human narcolepsy. Ann Neurol. 2001;50(3):381-8.

74. Challet E, Pévet P, Malan A. Effect of prolonged fasting and subsequent refeeding on freerunning rhythms of temperature and locomotor activity in rats. Behav Brain Res. 1997;84(1-2):275-

84.

75. Patterson KD, Pyle GF. The geography and mortality of the 1918 influenza pandemic. Bull Hist Med. 1991;65(1):4-21.

76. Gil Cuesta J, Aavitsland P, Englund H, Gudlaugsson O, Hauge SH, Lyytikainen O, et al. Pandemic vaccination strategies and influenza severe outcomes during the influenza $A(H 1 N 1) p d m 09$ pandemic and the post-pandemic influenza season: the Nordic experience. Euro Surveill. 2016;21(16).

77. Castilla J, Moran J, Martinez-Artola V, Reina G, Martinez-Baz I, Garcia Cenoz M, et al. Effectiveness of trivalent seasonal and monovalent influenza A(H1N1)2009 vaccines in population with major chronic conditions of Navarre, Spain: 2010/11 mid-season analysis. Euro Surveill. 2011;16(7):19799.

78. Läkemedelsverket. Occurrence of narcolepsy with cataplexy among children and adolescents in relation to the $\mathrm{H} 1 \mathrm{~N} 1$ pandemic and Pandemrix vaccinations: results of a case inventory study by the MPA in Sweden during 2009- 2010. MPA Rep 2011.

79. World Health Organisation. WHOQOL: Measuring Quality of Life. 2016 https://www.who.int/healthinfo/survey/whoqol-qualityoflife/en/. Accessed December 20, 2019. 80. Ozaki A, Inoue Y, Hayashida K, Nakajima T, Honda M, Usui A, et al. Quality of life in patients with narcolepsy with cataplexy, narcolepsy without cataplexy, and idiopathic hypersomnia without long sleep time: comparison between patients on psychostimulants, drug-naive patients and the general Japanese population. Sleep Med. 2012;13(2):200-6.

81. Ozaki A, Inoue $Y$, Nakajima T, Hayashida K, Honda M, Komada Y, et al. Health-related quality of life among drug-naive patients with narcolepsy with cataplexy, narcolepsy without cataplexy, and idiopathic hypersomnia without long sleep time. J Clin Sleep Med. 2008;4(6):572-8.

82. Inocente CO, Gustin MP, Lavault S, Guignard-Perret A, Raoux A, Christol N, et al. Quality of life in children with narcolepsy. CNS Neurosci Ther. 2014;20(8):763-71.

83. Szakács A, Chaplin JE, Tideman P, Strömberg U, Nilsson J, Darin N, et al. A population-based and case-controlled study of children and adolescents with narcolepsy: Health-related quality of life, adaptive behavior and parental stress. Eur J Paediatr Neurol. 2019;23(2):288-95.

84. Stores G, Montgomery P, Wiggs L. The psychosocial problems of children with narcolepsy and those with excessive daytime sleepiness of uncertain origin. Pediatrics. 2006;118(4):e1116-23.

85. Dorris L, Zuberi SM, Scott N, Moffat C, McArthur I. Psychosocial and intellectual functioning in childhood narcolepsy. Dev Neurorehabil. 2008;11(3):187-94.

86. Inocente CO, Gustin MP, Lavault S, Guignard-Perret A, Raoux A, Christol N, et al. Depressive feelings in children with narcolepsy. Sleep Med. 2014;15(3):309-14.

87. Liu X, Buysse DJ, Gentzler AL, Kiss E, Mayer L, Kapornai K, et al. Insomnia and Hypersomnia Associated with Depressive Phenomenology and Comorbidity in Childhood Depression. Sleep. 2007;30(1):83-90.

88. Szklo-Coxe M, Young T, Finn L, Mignot E. Depression: relationships to sleep paralysis and other sleep disturbances in a community sample. J Sleep Res. 2007;16(3):297-312.

89. Armitage R, Hoffmann R, Emslie G, Rintelman J, Moore J, Lewis K. Rest-activity cycles in childhood and adolescent depression. J Am Acad Child Adolesc Psychiatry. 2004;43(6):761-9.

90. Konofal E, Lecendreux M, Cortese S. Sleep and ADHD. Sleep Med Clin. 2010;11(7):652-8.

91. Daniels LE. Narcolepsy. Medicine. 1934;13(1):1-122.

92. Roy A. Psychiatric aspects of narcolepsy. The British Journal of Psychiatry. 1976;128(6):562-5.

93. Fortuyn HA, Mulders PC, Renier WO, Buitelaar JK, Overeem S. Narcolepsy and psychiatry: an evolving association of increasing interest. Sleep Med. 2011;12(7):714-9. 
94. Lecendreux M, Lavault S, Lopez R, Inocente CO, Konofal E, Cortese S, et al. AttentionDeficit/Hyperactivity Disorder (ADHD) Symptoms in Pediatric Narcolepsy: A Cross-Sectional Study. Sleep. 2015;38(8):1285-95.

95. Szakacs A, Hallbook T, Tideman P, Darin N, Wentz E. Psychiatric comorbidity and cognitive profile in children with narcolepsy with or without association to the H1N1 influenza vaccination. Sleep. 2015;38(4):615-21.

96. Modestino EJ, Winchester J. A retrospective survey of childhood ADHD symptomatology among adult narcoleptics. Journal of attention disorders. 2013;17(7):574-82.

97. Jennum P, Ibsen R, Knudsen S, Kjellberg J. Comorbidity and mortality of narcolepsy: a controlled retro- and prospective national study. Sleep. 2013;36(6):835-40.

98. Black J, Reaven NL, Funk SE, McGaughey K, Ohayon MM, Guilleminault C, et al. Medical comorbidity in narcolepsy: findings from the Burden of Narcolepsy Disease (BOND) study. Sleep Med. 2017;33:13-8.

99. Martinez-Orozco FJ, Vicario JL, Villalibre-Valderrey I, De Andres C, Fernandez-Arquero M, Peraita-Adrados R. Narcolepsy with cataplexy and comorbid immunopathological diseases. J Sleep Res. 2014;23(4):414-9.

100. Barateau L, Lopez R, Arnulf I, Lecendreux M, Franco P, Drouot X, et al. Comorbidity between central disorders of hypersomnolence and immune-based disorders. Neurology. 2017;88(1):93-100.

101. Dahmen N, Bierbrauer J, Kasten M. Increased prevalence of obesity in narcoleptic patients and relatives. Eur Arch Psychiatry Clin Neurosci. 2001;251(2):85-9.

102. Ferguson AV, Samson WK. The orexin/hypocretin system: a critical regulator of neuroendocrine and autonomic function. Front Neuroendocrinol. 2003;24(3):141-50.

103. Samson WK, Gosnell B, Chang JK, Resch ZT, Murphy TC. Cardiovascular regulatory actions of the hypocretins in brain. Brain Res. 1999;831(1-2):248-53.

104. Grimaldi D, Calandra-Buonaura G, Provini F, Agati P, Pierangeli G, Franceschini C, et al. Abnormal sleep-cardiovascular system interaction in narcolepsy with cataplexy: effects of hypocretin deficiency in humans. Sleep. 2012;35(4):519-28.

105. Longstreth Jr W, Koepsell TD, Ton TG, Hendrickson AF, Van Belle G. The epidemiology of narcolepsy. Sleep. 2007;30(1):13-26.

106. Poli F, Plazzi G, Di Dalmazi G, Ribichini D, Vicennati V, Pizza F, et al. Body mass indexindependent metabolic alterations in narcolepsy with cataplexy. Sleep. 2009;32(11):1491-7. 107. Kok S, Meinders A, Overeem S, Lammers G, Roelfsema F, Frolich M, et al. Reduction of plasma leptin levels and loss of its circadian rhythmicity in hypocretin (orexin)-deficient narcoleptic humans. The Journal of Clinical Endocrinology \& Metabolism. 2002;87(2):805-9.

108. Kotagal S, Krahn LE, Slocumb N. A putative link between childhood narcolepsy and obesity. Sleep Med. 2004;5(2):147-50.

109. Alam I, Lewis K, Stephens J, Baxter J. Obesity, metabolic syndrome and sleep apnoea: all proinflammatory states. Obes Rev. 2007;8(2):119-27.

110. Gao X, Schwarzschild MA, Wang $\mathrm{H}$, Ascherio A. Obesity and restless legs syndrome in men and women. Neurology. 2009;72(14):1255-61.

111. Lammers GJ, Pijl H, lestra J, Langius JA, Buunk G, Meinders AE. Spontaneous food choice in narcolepsy. Sleep. 1996;19(1):75-6.

112. van Holst RJ, van der Cruijsen L, van Mierlo P, Lammers GJ, Cools R, Overeem S, et al. Aberrant food choices after satiation in human orexin-deficient narcolepsy type 1 . Sleep. 2016;39(11):1951-9.

113. Maness C, Saini P, Bliwise DL, Olvera V, Rye DB, Trotti LM. Systemic exertion intolerance disease/chronic fatigue syndrome is common in sleep centre patients with hypersomnolence: $A$ retrospective pilot study. J Sleep Res. 2018:e12689.

114. Cremaschi RC, Hirotsu C, Tufik S, Coelho FM. High fatigue frequency in narcolepsy type 1 and type 2 in a Brazilian Sleep Center. Sleep Med. 2018. 
115. Knudsen S, Biering-Sorensen B, Kornum BR, Petersen ER, Ibsen JD, Gammeltoft S, et al. Early IVIg treatment has no effect on post-H1N1 narcolepsy phenotype or hypocretin deficiency.

Neurology. 2012;79(1):102-3.

116. Dauvilliers Y, Carlander B, Rivier F, Touchon J, Tafti M. Successful management of cataplexy with intravenous immunoglobulins at narcolepsy onset. Ann Neurol. 2004;56(6):905-8.

117. Mignot E, Nishino S. Emerging therapies in narcolepsy-cataplexy. Sleep. 2005;28(6):754-63.

118. Rogers $\mathrm{AE}$, Aldrich MS, Lin X. A comparison of three different sleep schedules for reducing daytime sleepiness in narcolepsy. Sleep. 2001;24(4):385-91.

119. Blackwell JE, Alammar HA, Weighall AR, Kellar I, Nash HM. A systematic review of cognitive function and psychosocial well-being in school-age children with narcolepsy. Sleep Med Rev. 2017;34:82-93.

120. Barateau L, Lopez R, Dauvilliers Y. Treatment options for narcolepsy. CNS drugs. 2016;30(5):369-79.

121. Mitler MM, Harsh J, Hirshkowitz M, Guilleminault C. Long-term efficacy and safety of modafinil (PROVIGIL((R))) for the treatment of excessive daytime sleepiness associated with narcolepsy. Sleep Med. 2000;1(3):231-43.

122. Dauvilliers Y, Bassetti C, Lammers GJ, Arnulf I, Mayer G, Rodenbeck A, et al. Pitolisant versus placebo or modafinil in patients with narcolepsy: a double-blind, randomised trial. Lancet Neurol. 2013;12(11):1068-75.

123. Mignot EJ. A practical guide to the therapy of narcolepsy and hypersomnia syndromes. Neurotherapeutics. 2012;9(4):739-52.

124. Mitler MM, Aldrich MS, Koob GF, Zarcone VP. Narcolepsy and its treatment with stimulants. ASDA standards of practice. Sleep. 1994;17(4):352-71.

125. Lin JS, Dauvilliers Y, Arnulf I, Bastuji H, Anaclet C, Parmentier R, et al. An inverse agonist of the histamine $\mathrm{H}(3)$ receptor improves wakefulness in narcolepsy: studies in orexin-/- mice and patients. Neurobiol Dis. 2008;30(1):74-83.

126. Lecendreux M, Poli F, Oudiette D, Benazzouz F, Donjacour CEHM, Franceschini C, et al. Tolerance and Efficacy of Sodium Oxybate in Childhood Narcolepsy with Cataplexy: A Retrospective Study. Sleep. 2012;35(5):709-11.

127. M $\varnothing$ ller LR, $\varnothing$ stergaard JR. Treatment with venlafaxine in six cases of children with narcolepsy and with cataplexy and hypnagogic hallucinations. Journal of child adolescent psychopharmacology. 2009;19(2):197-201.

128. Heier MS, Evsiukova T, Vilming S, Gjerstad MD, Schrader H, Gautvik K. CSF hypocretin-1 levels and clinical profiles in narcolepsy and idiopathic CNS hypersomnia in Norway. Sleep. 2007;30(8):96973.

129. Berry RB BR, Gamaldo CE, Harding SM, Lloyd RM, Marcus CL and Vaughn BV The AASM Manual for the Scoring of Sleep and Associated Events: Rules,Terminology and Technical Specifications, Version 2.2: American Academy of Sleep Medicine.; 2015.

130. Cole TJ, Lobstein T. Extended international (IOTF) body mass index cut-offs for thinness, overweight and obesity. Pediatr Obes. 2012;7(4):284-94.

131. Mancia G, Fagard R, Narkiewicz K, Redon J, Zanchetti A, Bohm M, et al. 2013 ESH/ESC Guidelines for the management of arterial hypertension: the Task Force for the management of arterial hypertension of the European Society of Hypertension (ESH) and of the European Society of Cardiology (ESC). J Hypertens. 2013;31(7):1281-357.

132. Medicine BCo. https://www.bcm.edu/bodycomplab/Flashapps/BPVAgeChartpage.html. Accessed December 13, 2019.

133. Maneesriwongul W, Dixon JK. Instrument translation process: a methods review. J Adv Nurs. 2004;48(2):175-86.

134. Varni JW, Burwinkle TM, Katz ER, Meeske K, Dickinson P. The PedsQL in pediatric cancer: reliability and validity of the Pediatric Quality of Life Inventory Generic Core Scales, Multidimensional Fatigue Scale, and Cancer Module. Cancer. 2002;94(7):2090-106. 
135. Varni JW, Seid M, Rode CA. The PedsQL: measurement model for the pediatric quality of life inventory. Med Care. 1999;37(2):126-39.

136. Varni JW, Burwinkle TM, Szer IS. The PedsQL Multidimensional Fatigue Scale in pediatric rheumatology: reliability and validity. J Rheumatol. 2004;31(12):2494-500.

137. Ervik S, Abdelnoor M, Heier MS, Ramberg M, Strand G. Health-related quality of life in narcolepsy. Acta Neurol Scand. 2006;114(3):198-204.

138. Dodel R, Peter H, Walbert T, Spottke A, Noelker C, Berger K, et al. The socioeconomic impact of narcolepsy. Sleep. 2004;27(6):1123-8.

139. Ingravallo F, Gnucci V, Pizza F, Vignatelli L, Govi A, Dormi A, et al. The burden of narcolepsy with cataplexy: how disease history and clinical features influence socio-economic outcomes. Sleep Med. 2012;13(10):1293-300.

140. Morgenthaler TI, Kapur VK, Brown TM, Swick TJ, Alessi C, Aurora RN, et al. Practice parameters for the treatment of narcolepsy and other hypersomnias of central origin. 2007;30(12):1705-11.

141. Rocca FL, Finotti E, Pizza F, Ingravallo F, Gatta M, Bruni O, et al. Psychosocial Profile and Quality of Life in Children With Type 1 Narcolepsy: A Case-Control Study. Sleep. 2016;39(7):1389-98.

142. Weaver TE, Cuellar N. A randomized trial evaluating the effectiveness of sodium oxybate therapy on quality of life in narcolepsy. Sleep. 2006;29(9):1189-94.

143. Lammers G, Arends J, Declerck A, Ferrari M, Schouwink G, Troost J. Gammahydroxybutyrate and narcolepsy: a double-blind placebo-controlled study. Sleep. 1993;16(3):216-20.

144. Black J, Pardi D, Hornfeldt CS, Inhaber N. The nightly administration of sodium oxybate results in significant reduction in the nocturnal sleep disruption of patients with narcolepsy. Sleep Med. 2009;10(8):829-35.

145. Bogan RK, Roth T, Schwartz J, Miloslavsky M. Time to response with sodium oxybate for the treatment of excessive daytime sleepiness and cataplexy in patients with narcolepsy. Journal of clinical sleep medicine : JCSM : official publication of the American Academy of Sleep Medicine. 2015;11(4):427-32.

146. Torsheim T, Wold B. School-related stress, school support, and somatic complaints: A general population study. Journal of Adolescent Research. 2001;16(3):293-303.

147. American Psychiatric Association. Diagnostic and statistical manual of mental disorders. 5th ed. Arlington VA:: American Psychiatric Assosiation; 2013.

148. Dahmen N, Querings K, Grun B, Bierbrauer J. Increased frequency of migraine in narcoleptic patients. Neurology. 1999;52(6):1291-3.

149. Dauvilliers Y, Bayard S, Shneerson JM, Plazzi G, Myers AJ, Garcia-Borreguero D. High pain frequency in narcolepsy with cataplexy. Sleep Med. 2011;12(6):572-7.

150. Ruoff CM, Reaven NL, Funk SE, McGaughey KJ, Ohayon MM, Guilleminault C, et al. High Rates of Psychiatric Comorbidity in Narcolepsy: Findings From the Burden of Narcolepsy Disease (BOND) Study of 9,312 Patients in the United States. J Clin Psychiatry. 2017;78(2):171-6.

151. Broughton R, Ghanem Q, Hishikawa $Y$, Sugita $Y$, Nevsimalova S, Roth B. Life effects of narcolepsy in 180 patients from North America, Asia and Europe compared to matched controls. Can J Neurol Sci. 1981;8(4):299-304.

152. Calhoun SL, Vgontzas AN, Fernandez-Mendoza J, Mayes SD, Tsaoussoglou M, Basta M, et al. Prevalence and risk factors of excessive daytime sleepiness in a community sample of young children: the role of obesity, asthma, anxiety/depression, and sleep. Sleep. 2011;34(4):503-7.

153. Krahn LE. Psychiatric disorders associated with disturbed sleep. Semin Neurol. 2005;25(01):90-6.

154. Hayter SM, Cook MC. Updated assessment of the prevalence, spectrum and case definition of autoimmune disease. Autoimmunity reviews. 2012;11(10):754-65.

155. Wang Z, Wu H, Stone WS, Zhuang J, Qiu L, Xu X, et al. Body weight and basal metabolic rate in childhood narcolepsy: a longitudinal study. Sleep Med. 2016;25:139-44.

156. Steinberger J, Daniels SR. Obesity, insulin resistance, diabetes, and cardiovascular risk in children: an American Heart Association scientific statement from the Atherosclerosis, Hypertension, 
and Obesity in the Young Committee (Council on Cardiovascular Disease in the Young) and the Diabetes Committee (Council on Nutrition, Physical Activity, and Metabolism). Circulation. 2003;107(10):1448-53.

157. Varni JW, Burwinkle TM, Katz ER, Meeske K, Dickinson P. The PedsQL ${ }^{\mathrm{TM}}$ in pediatric cancer: reliability and validity of the pediatric quality of life inventory ${ }^{\mathrm{TM}}$ generic core scales, multidimensional fatigue scale, and cancer module. Cancer. 2002;94(7):2090-106.

158. Haack M, Mullington JM. Sustained sleep restriction reduces emotional and physical wellbeing. Pain. 2005;119(1-3):56-64.

159. Harvey SB, Wessely S, Kuh D, Hotopf $M$. The relationship between fatigue and psychiatric disorders: evidence for the concept of neurasthenia. J Psychosom Res. 2009;66(5):445-54.

160. Brundin L, Petersen A, Bjorkqvist M, Traskman-Bendz L. Orexin and psychiatric symptoms in suicide attempters. J Affect Disord. 2007;100(1-3):259-63.

161. Chen $\mathrm{Q}$, de Lecea L, Hu Z, Gao D. The hypocretin/orexin system: an increasingly important role in neuropsychiatry. Med Res Rev. 2015;35(1):152-97.

162. Winsky-Sommerer R, Yamanaka A, Diano S, Borok E, Roberts AJ, Sakurai T, et al. Interaction between the corticotropin-releasing factor system and hypocretins (orexins): a novel circuit mediating stress response. J Neurosci. 2004;24(50):11439-48.

163. Feng P, Vurbic D, Wu Z, Hu Y, Strohl KP. Changes in brain orexin levels in a rat model of depression induced by neonatal administration of clomipramine. Journal of psychopharmacology (Oxford, England). 2008;22(7):784-91.

164. Pizza F, Peltola H, Sarkanen T, Moghadam KK, Plazzi G, Partinen M. Childhood narcolepsy with cataplexy: comparison between post-H1N1 vaccination and sporadic cases. Sleep Med.

2014;15(2):262-5.

165. Sarkanen T, Alakuijala A, Partinen M. Clinical course of H1N1-vaccine-related narcolepsy. Sleep Med. 2016;19:17-22.

166. Indrayan A. Medical biostatistics: Chapman and Hall/CRC; 2012.

167. Juliusson PB, Hjelmesaeth J, Bjerknes R, Roelants M. New curves for body mass index among children and adolescents. Tidsskr Nor Laegeforen. 2017;137(18).

168. Littner MR, Kushida C, Wise M, G. Davila D, Morgenthaler T, Lee-Chiong T, et al. Practice parameters for clinical use of the multiple sleep latency test and the maintenance of wakefulness test. Sleep. 2005;28(1):113-21.

169. Janssen KC, Phillipson S, O'Connor J, Johns MW. Validation of the Epworth Sleepiness Scale for children and adolescents using Rasch analysis. Sleep medicin. 2017;33:30-5.

170. Anic-Labat S, Guilleminault C, Kraemer HC, Meehan J, Arrigoni J, Mignot E. Validation of a cataplexy questionnaire in 983 sleep-disorders patients. Sleep. 1999;22(1):77-87.

171. Murali H, Kotagal S. Off-Label Treatment of Severe Childhood Narcolepsy-Cataplexy With Sodium Oxybate. Sleep. 2006;29(8):1025-9.

172. Reinfjell T, Diseth TH, Veenstra M, Vikan A. Measuring health-related quality of life in young adolescents: reliability and validity in the Norwegian version of the Pediatric Quality of Life Inventory 4.0 (PedsQL) generic core scales. Health and quality of life outcomes. 2006;4:61.

173. Patrick DL, Deyo RA. Generic and disease-specific measures in assessing health status and quality of life. Med Care. 1989;27(3 Suppl):S217-32.

174. Novik TS. Validity of the Child Behaviour Checklist in a Norwegian sample. Eur Child Adolesc Psychiatry. 1999;8(4):247-54.

175. Salbach-Andrae H, Lenz K, Lehmkuhl U. Patterns of agreement among parent, teacher and youth ratings in a referred sample. Eur Psychiatry. 2009;24(5):345-51.

176. Salbach-Andrae H, Klinkowski N, Lenz K, Lehmkuhl U. Agreement between youth-reported and parent-reported psychopathology in a referred sample. Eur Child Adolesc Psychiatry. 2009;18(3):136-43.

177. Achenbach TM. Manual for the child behavior checklist/4-18 and 1991 profile. University of Vermont, Departement of Psychiatry, Burlington1991. 
178. Achenbach TM, McConaughy SH, Howell CT. Child/adolescent behavioral and emotional problems: implications of cross-informant correlations for situational specificity. Psychol Bull. 1987;101(2):213-32. 
PAPERS I-III 

I 

Original Article

\title{
Changes in quality of life in individuals with narcolepsy type 1 after the H1N1-influenza epidemic and vaccination campaign in Norway: a two-year prospective cohort study
}

\author{
Sebjørg Hesla Nordstrand a, b, *, Berit Hjelde Hansen a, b, c, Yusman Kamaleri ${ }^{\text {d, }}$, \\ Kristian Bernhard Nilsen ${ }^{\text {e, f }}$, Terje Rootwelt ${ }^{\text {b, g }}{ }^{\text {, Tor-Ivar Karlsen }}{ }^{\text {h }}$, Stine Knudsen ${ }^{\text {a }}$ \\ ${ }^{a}$ Norwegian Centre of Expertise for Neurodevelopmental Disorders and Hypersomnias, Department of Rare Disorders, Oslo University Hospital, Norway \\ ${ }^{\mathrm{b}}$ Faculty of Medicine, University of Oslo, Norway \\ ${ }^{\mathrm{c}}$ Division of Mental Health Akershus University Hospital, Lorenskog, Norway \\ ${ }^{\mathrm{d}}$ National Health Quality Registries, Oslo University Hospital, Norway \\ e Department of Neuromedicine and movement science, Faculty of Medicine, Norwegian University of Science and Technology, Trondheim, Norway \\ ${ }^{\mathrm{f}}$ Department of Neurology, Section for Clinical Neurophysiology, Oslo University Hospital, Ullevål, Norway \\ ${ }^{g}$ Department of Paediatrics, Oslo University Hospital, Norway \\ ${ }^{\mathrm{h}}$ Faculty of Health and Sports Sciences, University of Agder, Norway
}

\section{A R T I C L E I N F O}

\section{Article history:}

Received 15 January 2018

Received in revised form

11 May 2018

Accepted 25 May 2018

Available online 27 June 2018

\section{Keywords:}

Quality of life

Narcolepsy

Pediatric

H1N1

Pediatrics comorbidity

\begin{abstract}
A B S T R A C T
Objective: Cross-sectional studies show a lower health-related quality of life (HRQoL) in individuals with narcolepsy. We aimed to describe changes in HRQoL after two years of multidisciplinary follow-up in a cohort of mainly post-H1N1 vaccination narcolepsy type-1 (NT1) patients in Norway.

Methods: Prospective-cohort study. Narcolepsy diagnosis was based on the International Classification of Sleep Disorders (third edition). Psychiatric comorbidity was assessed using the Achenbach System of Empirically Based Assessment (ASEBA). HRQoL was evaluated with the Pediatric Quality of Life Inventory (PedsQL TM Generic Core Scales 4.0) at baseline and follow-up. Mean follow-up time was 20.7 (2.7) months. Results: Thirty one patients (18 females) with NT1, mean age 14.6 (SD $=4.8$ ) years answered questionnaires at baseline and follow-up. On a group level, the PedsQL Total Health Summary score significantly improved by a mean of $5.9(95 \% \mathrm{CI}=0.4,11.9), \mathrm{p}=0.038$; this was mainly driven by improvements in the Physical Health Summary score by $9.8(3.0,16.5)$ points, $\mathrm{p}=0.006$ and the School Functioning Scale score by $7.5(1.0,13.9)$ points $\mathrm{p}=0.025$. The Total ASEBA score was correlated with PedsQL Total Health Summary score at baseline, but not with changes in HRQoL. Sodium oxybate $\left(\right.$ Xyrem $\left.{ }^{\circledR}\right)$ treatment at follow up was positively associated with changes in PedsQL Total Health Summary score, after adjusting for age and gender, $\mathrm{p}=0.027$.

Conclusion: HRQoL in NT1 patients improved after two years of follow-up. The use of sodium oxybate (Xyrem ${ }^{\circledR}$ ) at follow-up was associated with increases in HRQoL. Psychiatric comorbidity was correlated with HRQoL at baseline but did not predict changes in HRQoL at follow-up.
\end{abstract}

(c) 2018 Elsevier B.V. All rights reserved.

\section{Introduction}

Narcolepsy is a chronic disabling sleep disorder characterized by instability of the wake-sleep cycle, REM-sleep and muscle tone regulation [1,2]. Narcolepsy is divided into narcolepsy type 1 (NT1) and narcolepsy type 2 (NT2). The onset of NT1 commonly occurs

\footnotetext{
* Corresponding author. Norwegian Centre of Expertise for Neurodevelopmental Disorders and Hypersomnias, Department of Rare Disorders, Oslo University Hospital, Norway.

E-mail addresses: sebnor@ous-hf.no, sebnords@online.no (S.H. Nordstrand).
}

during adolescence or early adulthood, and its clinical presentation may vary with age $[3,4]$.

The typical clinical symptoms of NT1 are excessive daytime sleepiness (EDS), hypnagogic/hypnopompic hallucination ( $\mathrm{HH})$, sleep paralysis (SP), cataplexy and fragmented nocturnal sleep [1]. Children might present with somewhat different symptoms and can initially show paradoxical hyperactivity and/or irritability, atypical cataplexy and precocious puberty [5-8]. NT1 often has considerable negative consequences for individuals diagnosed with the disease. Typical problems include difficulties in obtaining an education or keeping a job, resulting in negative 
socioeconomic consequences and reduced health-related quality of life [8-13].

The hypocretin system is central to the pathogenesis of NT1, but also affects other important brain functions and signaling systems as it projects widely through the brain [14,15]. Recently, hypocretin has been hypothesized to play a role in several neuropsychiatric disorders such as depression, anxiety, posttraumatic stress disorder and schizophrenia [14]. In line with this, several studies have shown increased behavioral problems and psychiatric comorbidity among patients with NT1 [16]. Internalized problems such as depression and anxiety are seen in $20-30 \%$ of patients with narcolepsy $[9,17,18]$. Externalized problems, like Attention Deficit/Hyperactivity Disorder (ADHD), are 8-15 times more frequent in NT1 compared to the general population [7,19]. Moreover, Inocente et al., [20], found depression to be the factor that most strongly affects the quality of life in narcolepsy patients, further demonstrating the importance of measuring psychiatric comorbidity in this population.

Following the 2009 H1N1 epidemic and vaccination campaign with Pandemrix ${ }^{\circledR}$, there was an abrupt increase in NT1 incidence, especially among children and young adults in Norway and several other countries [21-24]. Whether or not the increase was a direct consequence of the vaccine is yet to be proved [25]. NT1 diagnosed subsequent to the Pandemrix vaccine, in addition to classic NT1symptoms, has been associated with symptoms indicating behavioral problems in almost half of patients $[22,26]$. The high occurrence of psychiatric comorbidity may indicate a more severe phenotype; this has been questioned by Pizza et al., [27].

Several cross-sectional studies showed an impaired healthrelated quality of life (HRQoL) in individuals with narcolepsy $[12,28-34]$, but, to our knowledge, only two prospective-cohort studies have addressed this matter. Vignatelli et al., [35] studied an adult narcolepsy population, and Sarkanen et al., [36] studied Finnish children and adolescents. Both reported a low HRQoL at baseline that was unchanged at follow-up. The primary aim of the present study was to use the PedsQL questionnaire to prospectively study changes in HRQoL over time in a Norwegian cohort of mainly post-H1N1 vaccination NT1 patients. The secondary aim was to use the ASEBA questionnaires to investigate possible associations between psychiatric comorbidity and HRQoL and, to evaluate whether psychiatric comorbidity can predict changes in HRQoL. Furthermore, we wanted to determine whether sodium oxybate or severity of disease burden measured by Epworth Sleepiness Scale (ESS), can predict of changes in HRQoL since our clinical impression is that these three variables strongly influence HRQoL outcomes. The identification of possible predictors for changes in HRQOL may be important for developing better clinical approaches for young patients with narcolepsy.

\section{Methods}

\subsection{Participants}

In December 2014, 91 individuals identified as our national cohort of narcolepsy patients with onset after autumn 2009 were invited to participate in the study and were sent baseline questionnaires. The study was approved by the Regional Committees for Medical and Health Research Ethics (REK) (REK-number 2014/450). Inclusion criteria were: age, 5-59years, the onset of narcolepsy symptoms after autumn 2009, and narcolepsy diagnosis based on the International Classification of Sleep Disorders (third edition) (ICSD-3) [1]. Written informed consent was obtained before inclusion.

During the first quarter of 2015, 42 of the 91 patients had returned their baseline questionnaires; and 36 of the questionnaires were complete. After a full diagnostic evaluation at our tertiary specialist service center, the Norwegian Center of Expertise for Neurodevelopmental Disorders and Hypersomnias (NevSom), three of the 36 patients were excluded because they did not fulfill the inclusion criteria. One patient presented possible debut of symptoms immediately before vaccination with Pandemrix ${ }^{\circledR}$. However, since these were typical core NT1 symptoms, the patient was not excluded from this study.

\subsection{Procedure}

Participants and their first-degree relatives stayed at NevSom for 2-4 days. They were informed about narcolepsy and provided kindergarten/school/work support by a specialized team consisting of a senior consulting neurologist specializing in sleep medicine, a specialist nurse and/or specialist educator, and in the case of patients younger than 20 years, a child psychiatrist. All participants underwent overnight polysomnography (PSG) followed by a multiple sleep latency test (MSLT) with five nap opportunities. Their circadian rhythm was monitored for 10-14 days before an investigation with actigraphs (Actiwatch Spectrum Plus, Phillips). Fasting blood tests were done to establish HLA-typing and to control for routine blood parameters. A lumbar puncture to sample cerebrospinal fluid (CSF) was performed at local hospitals and hypocretin-1 in CSF was measured at the Hormone Laboratory, Department of Medical Biochemistry, Oslo University Hospital, by previously described methods [37].

Follow-up questionnaires were e-mailed to the 33 study participants who had completed the baseline questionnaires; and 31 of these participants returned the follow-up questionnaire. Participants received the follow-up questionnaire before being interviewed by telephone (beginning of September 2016 to the end of December 2016). In addition, participants were asked about their current medications, and if they had sought medical care, or been diagnosed with disorders other than narcolepsy, after being included in our study. For participants younger than 12 years, or for other reasons requested by the participant, a parent was also present and able to contribute to the telephone interview.

\subsection{Narcolepsy questionnaires}

Subjective core narcolepsy symptoms (cataplexy, EDS, HH, SP) were assessed with the Stanford Sleep Questionnaire (SSQ), including the Epworth Sleepiness Scale (ESS), giving scores ranging from 0 to 24 [38].

\subsection{Medication}

Pharmacological treatment was evaluated, and appropriate changes were made during the multidisciplinary treatment in our specialist center. All study participants took no medication 14 days before, and during the stay.

\subsection{Questionnaires}

\subsubsection{Health-related quality of life questionnaires}

HRQoL was assessed using the 23-item PedsQL ${ }^{\text {TM }}$ Generic Core Scales 4.0 questionnaires which can be divided into the following Functioning scales: Physical (eight items); Emotional (five items); Social (five items); and School (five items) [39]. The self-reported version is available for ages $5-7,8-12,13-18,18-25$ and $>26$ years. The Norwegian version of the questionnaires are translated for all age groups and validated for the 5-7, 8-12 and 13-18 age groups [40]. The items are essentially identical, differing only in developmental-linguistic age-appropriateness. The items relate to how much of a problem the respondent has experienced in the previous month on a five-point Likert scale $(0=$ never a problem, 1 = almost never a problem, $2=$ sometimes a problem, $3=$ often a problem, 4 = almost always a problem). According to the scoring 
instructions (https://www.pedsql.org/score.html) items are reverse-scored and linearly transformed to a 0 to 100 scale $(0=100$, $1=75,2=50,3=25,4=0$ ), where higher scores indicate better HRQoL. Scale scores are computed as their sum divided by the number of items scored. In addition to the four Functioning scales, three Summary scores were computed. The Physical Health Summary score is the same as the result for the Physical Functioning scale. The Psychosocial Health Summary score (15 items) is calculated by adding the items in the Emotional, Social and School Functioning scales divided by the number of items answered. Finally, the Total Health Summary score is calculated by adding the scores of the 23 items divided by the number of items answered, ensuring that missing data were accounted for [41].

Our study did not have a control group. However, due to a large ( $\mathrm{n}=425$ ), study that validated the PedsQL in healthy children and adolescents in Norway, our results can be compared to normative data [40].

\subsubsection{Psychiatric comorbidity questionnaires}

To assess psychiatric comorbidity we used the Norwegian version of the Achenbach System of Empirically Based Assessment (ASEBA) Child Behavior Checklist (CBCL) for the age ranges 1.5-5 and 6-18 years. The corresponding Adult Self Report (ASR) was used for patients aged $18-59$ years. ASEBA is a widely used tool for assessing psychiatric symptoms such as adaptive and maladaptive functioning, with comparable scales across age ranges $[42,43]$. The CBCL consists of 120 items divided into eight different syndrome scales: Anxious/depressed; Withdrawn/depressed; Somatic complaints; Social problems; Thought problems; Attention problems; Rule-breaking behavior; and Aggressive behavior. Each item is scored on a three-point response scale, where 0 means 'not true', 1 is 'somewhat or sometimes true' and 2 is 'very or often true'. Scores are the sums for each of the eight syndrome scales. Five of the syndrome scales for children and six for adults are also used to derive two broadband scales: an Internalizing score (Anxious/depressed, Withdrawn/depressed and Somatic complaints) and an Externalizing score (Rule-breaking behavior, Aggressive behavior and Intrusive behavior (adults)). The other syndrome scales, three for children and two for adults (Thought problems, Social problems (children) and Attention problems), are not considered to be components of either the Internalizing or Externalizing scores, but in conjunction with these latter are used to derive a Total score. Higher scores indicate poorer functioning. The ASR is equivalent to the CBCL, except that in ASR the Somatic complaints syndrome scale is replaced by that of Intrusive behavior. The raw scores of CBCL and ASR were manually scored by us and converted into T-scores to facilitate comparison. For the syndrome scales the normal range is considered to be a T-score below 65, borderline cases have scores from 65 to 70 , and clinical cases have values from 70 or higher. For the broadband scales (Internalizing, Externalizing and Total scores), the normal range is considered to be a T-score of less than 60 , borderline patients have scores between 60 and 63, and values of 64 and above are considered to be in the clinical range.

\subsection{Statistics}

Data were analyzed with IBM SPSS version 23. Data are presented as frequencies; mean (standard deviation (SD), 95\% confidence interval $(\mathrm{CI})$ ) or median (interquartile range $(\mathrm{IQR})$ ) are given when appropriate. To check for inclusion bias, comparison of the 31 participants with the rest of the remaining cohort $(n=84)$ was done by using the Fisher exact test. Change between PedsQL Total Health Summary score at baseline and follow-up was assessed with pairedsample T-tests. To assess whether those with low PedsQL Total Health Summary scores at baseline had increased their scores at follow-up, study participants were divided into two groups based on whether their PedsQL Total Health Summary score at baseline was less or greater than the mean PedsQL Total Health Summary score. Results of the two groups were then compared with an independent-sample T-test and by linear regression, adjusting for age and gender. Data from ASEBA were analyzed using Spearman's rank correlation, and linear regression was used when the ASEBA results were assessed for their predictive value for PedsQL, adjusting for age. Values of $\mathrm{p}<0.05$ were considered statistically significant.

\section{Results}

\subsection{Characteristics}

Demographic, clinical, and laboratory characteristics of the 31 participants (18 females) with NT1 who participated at both baseline and follow up, are presented in Table 1. All participants presented with typical clinical NT1 symptoms. Cataplexy was present in the two patients without available hypocretin-levels. Hypocretin-1 was low in the one patient with normal PSG/MSLT. All patients except for two were H1N1-vaccinated with Pandemrix. No sign of inclusion bias was detected, when comparing participants with the non-participating remaining larger cohort regarding core narcolepsy parameters (frequency of cataplexy, HH, SP, PSG/ MSLT (Total sleep time, mean sleep latency test, number of sleep onset REM), or hypocretin-1 deficiency (all $\mathrm{p} \geq 0.125$ )).

\subsection{Health-related quality of life}

The mean Total Health Summary score significantly improved from baseline to follow up by 5.9 points (95\% CI: $0.4,11.4$ ); this was mainly driven by improvements in the Physical Functioning by 9.8 $(3.0,16.5)$ points and the School Functioning scale by $7.5(1.0,13.9)$ points (Table 2). Comparison between those with a Total Health

\section{Table 1}

Demographic, clinical, laboratory characteristics and medications in children and young adults with narcolepsy type 1 after 2009 .

\begin{tabular}{|c|c|c|c|}
\hline Characteristics & & Mean (SD) & Frequency $(\mathrm{n} / \mathrm{N})$ \\
\hline Age (years) & & $14.6(4.8)$ & \\
\hline \multirow[t]{2}{*}{ Gender } & Female & & $18 / 31$ \\
\hline & Male & & $13 / 31$ \\
\hline \multirow[t]{5}{*}{ Symptoms } & EDS & & $31 / 31$ \\
\hline & Cataplexy & & $29 / 31$ \\
\hline & Hallucination & & $23 / 31$ \\
\hline & SP & & $20 / 31$ \\
\hline & ESS & $13.7(4.5)$ & \\
\hline \multirow[t]{3}{*}{ Body Mass Index } & Normal weight & & $12 / 31$ \\
\hline & Overweight & & $11 / 31$ \\
\hline & Obese & & $8 / 31$ \\
\hline \multirow[t]{2}{*}{ Pandemrix-vaccinated } & Yes & & $29 / 31$ \\
\hline & No & & $2 / 31$ \\
\hline \multirow[t]{2}{*}{ HLADQB1*0602 } & Positive & & $29 / 30$ \\
\hline & Negative & & $1 / 30$ \\
\hline \multirow[t]{3}{*}{ CSF Hypocretin-1 } & $>200 \mathrm{pg} / \mathrm{ml}$ & & $0 / 29$ \\
\hline & $110-200 \mathrm{pg} / \mathrm{ml}$ & & $2 / 29$ \\
\hline & $<110 \mathrm{pg} / \mathrm{ml}$ & & $27 / 29$ \\
\hline \multirow[t]{2}{*}{ PSG/MSLT } & Positive & & $30 / 31$ \\
\hline & Negative & & $1 / 31$ \\
\hline \multirow[t]{10}{*}{ Medication at baseline } & Methylphenidate & & $19 / 31$ \\
\hline & Modafinil & & $13 / 31$ \\
\hline & Venlafaxine & & $6 / 31$ \\
\hline & Fluoxetine & & $3 / 31$ \\
\hline & Sodium oxybate & & $11 / 31$ \\
\hline & Other & & $5 / 31$ \\
\hline & No medication & & $4 / 31$ \\
\hline & One medication & & $9 / 31$ \\
\hline & Two medication & & $13 / 31$ \\
\hline & Three medication & & $6 / 31$ \\
\hline
\end{tabular}

EDS, excessive daytime sleepiness; SP, sleep paralysis; ESS, Epworth Sleepiness Scale; CSF, cerebrospinal fluid; PSG, polysomnography; MSLT, Multiple Sleep Latency Test. 
Table 2

Scale description for Pediatric Quality of Life Inventory (PedsQL) at baseline (T1) and follow-up (T2).

\begin{tabular}{|c|c|c|c|c|}
\hline Self-reported PedsQL & T1 Mean (SD) & T2 Mean (SD) & T2-T1 Mean (95\% CI) & $\mathrm{p}$ \\
\hline \multicolumn{5}{|l|}{ Functioning scale score } \\
\hline Physical & $65.9(20.7)$ & $75.7(17.4)$ & $9.8(3.0,16.6)$ & $0.006^{*}$ \\
\hline Emotional & $63.2(25.6)$ & $65.8(21.9)$ & $2.6(-4.5,9.7)$ & 0.464 \\
\hline Social & $74.0(19.6)$ & $75.3(20.5)$ & $1.3(-5.6,8.1)$ & 0.703 \\
\hline School & $53.4(18.4)$ & $60.8(13.7)$ & $7.5(1.0,13.9)$ & $0.025^{*}$ \\
\hline \multicolumn{5}{|l|}{ Summary score } \\
\hline Psychosocial & $63.5(18.9)$ & $67.3(16.1)$ & $3.8(-1.7,9.3)$ & 0.171 \\
\hline Total Health & $64.4(18.6)$ & $70.2(16.1)$ & $5.9(0.3,11.4)$ & $0.038^{*}$ \\
\hline
\end{tabular}

T1, first time questionnaire was answered; T2, questionnaire answered at follow-up The mean (SD) scores from Reinfjell et al.'s study validating the PedsQL in children and adolescent in Norway; Physical Health score; 91 (10), Emotional Functioning score; 77 (17), Social Functioning score; 88 (13), School Functioning score; 78 (15), Psychosocial Health Summary score; 82 (13), and finally, Total Health Summary score; 85 (11) [40].

Table 3

Improvement in Pediatric Quality of Life Inventory (PedsQL) from baseline to follow-up in subgroups based on group mean PedsQL Total Health summary score at baseline.

\begin{tabular}{|c|c|c|c|c|}
\hline Changes in self-reported PedsQL & Less than group mean (SD) & Greater than group mean (SD) & Difference between Groups (95\% CI) & $\mathrm{p}$ \\
\hline \multicolumn{5}{|l|}{ Functioning scale scores } \\
\hline Physical & $13.6(24.1)$ & $6.7(12.0)$ & $6.9(-7.9,21.8)$ & 0.341 \\
\hline Emotional & $14.0(16.8)$ & $-6.7(16.4)$ & $20.7(8.4,33.0)$ & $0.002^{*}$ \\
\hline Social & $5.7(19.1)$ & $-2.4(18.0)$ & $8.1(-5.7,21.9)$ & 0.241 \\
\hline School & $15.2(15.2)$ & $1.1(13.5)$ & $14.1(1.5,26.7)$ & $0.030^{*}$ \\
\hline \multicolumn{5}{|l|}{ Summary scores } \\
\hline Psychosocial & $11.6(15.9)$ & $-2.7(11.0)$ & $14.3(3.9,24.5)$ & $0.009^{*}$ \\
\hline Total Health & $12.3(17.3)$ & $0.6(10.8)$ & $11.7(0.7,22.8)$ & $0.039 *$ \\
\hline
\end{tabular}

Less than the group mean, $\mathrm{n}=14$; greater than the group mean, $\mathrm{n}=17$.

Summary score lower or higher than the group mean clarifies a significant improvement in HRQoL at follow up, for patients who scored lower than the group mean at baseline, School and Emotional Functioning $14.1(1.5,26.6)$ and 20.7 points $(8.4,3.0)$ respectively, but not in Physical and Social Functioning at follow up, giving an increase in the Total Health Summary score of 11.7 points $(0.7,22.7)$ (Table 3$)$. This finding was strengthened after adjusting for age and gender. In summary, the mean Total Health Summary score significantly improved during follow-up (Fig. 1).

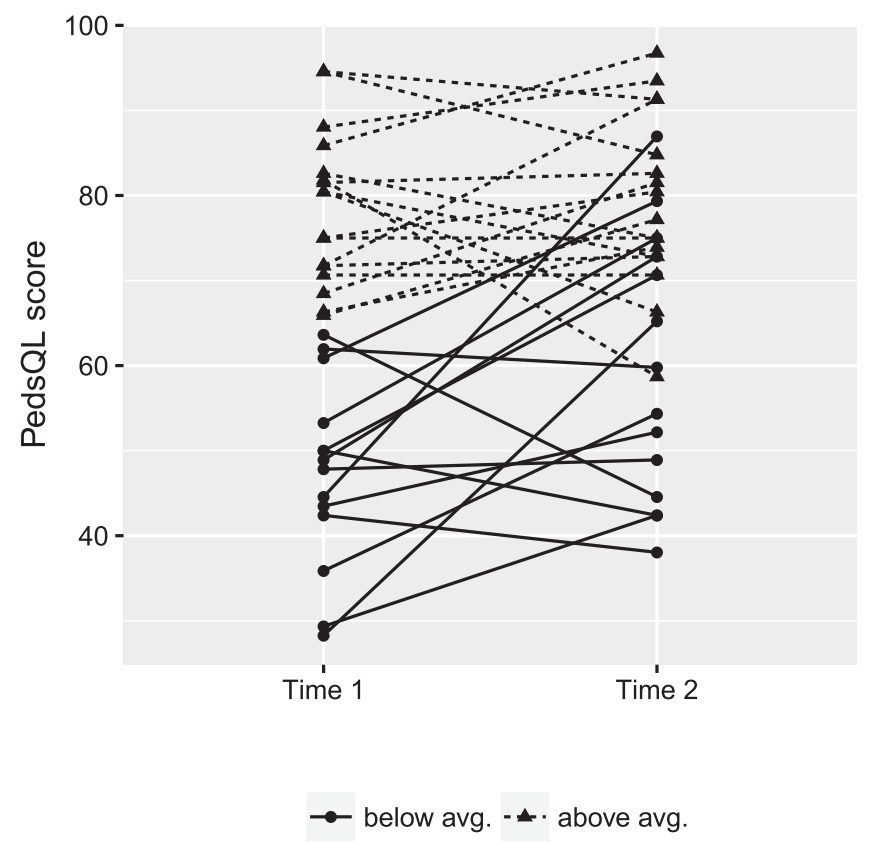

Fig. 1. Individual changes in Pediatric Quality of Life Inventory (PedsQL), Total Health Summary score, at baseline (T1) and follow-up (T2).

\subsection{Psychiatric comorbidity}

A total of $31.0 \%$ of patients had an ASEBA total score in the borderline or clinical range (Table 4 ).

\subsection{Correlations and possible predictors}

The use of sodium oxybate (Xyrem $\left.{ }^{\circledR}\right)$ at follow up was positively associated with changes in PedsQL after adjusting for age and gender (Table 5). When adding other narcolepsy medications (methylphenidate, modafinil, venlafaxine, and fluoxetine) to the linear regression model, sodium oxybate was still the only medication with statistical significance. ASEBA scores (Internalizing, Externalizing and Total scores) at baseline were significantly correlated with the PedsQL Total Health score at baseline (all $p<0.005$ ), but showed no significant predictive value for changes in PedsQL between baseline and follow-up (Table 5). ESS at baseline (as a measure of disease burden) was not correlated with any changes in HRQoL from baseline to follow up. Cataplexy severity (frequency) did not show any significant association with changes in PedsQL at follow-up (data not shown). In conclusion, no predictors of changes in PedsQL were found except the use of sodium oxybate $\left(\right.$ Xyrem $\left.{ }^{\circledR}\right)$ at follow up.

\section{Discussion}

The main finding in the current study of Norwegian NT1 patients who developed narcolepsy after the 2009 epidemic and vaccination campaign, was impaired HRQoL compared to national normative data $[40,44]$. We found a significant increase in HRQoL after two years of follow-up at a tertiary care center. At the group level, improvements were greatest in Physical Health and School Functioning. Further analysis showed that subjects with a below-average HRQoL score at baseline had improved Psychosocial Health scores as well. The use of sodium oxybate at follow up was positively associated with a better HRQoL outcome at follow up. Indicators of psychiatric comorbidity (ASEBA-scores) and narcolepsy disease burden 
Table 4

ASEBA T-score at baseline.

\begin{tabular}{|c|c|c|c|c|c|c|c|c|}
\hline \multirow[t]{2}{*}{ Broadband Scale } & \multicolumn{3}{|l|}{ ASR $\mathrm{n}=8$} & \multicolumn{3}{|l|}{ CBCL $n=23$} & \multicolumn{2}{|c|}{ ASR or CBCL $n=31$} \\
\hline & Mean (SD) & $\mathrm{BR} \%$ & $\mathrm{CR} \%$ & Mean (SD) & $\mathrm{BR} \%$ & $\mathrm{CR} \%$ & $\mathrm{BR} \%$ & $\mathrm{CR} \%$ \\
\hline Internalizing problems & $64.25(11.08)$ & 12,5 & 50 & $59.78(8.07)$ & 21.7 & 30.4 & 17.2 & 34.5 \\
\hline Externalizing problems & $55.38(7.73)$ & 25.0 & 12.5 & $52.70(9.47)$ & 8.7 & 13.0 & 13.8 & 10.3 \\
\hline Total problems & $60.25(8.58)$ & 0 & 50 & $57.43(8.40)$ & 4.3 & 26.1 & 3.4 & 31.0 \\
\hline
\end{tabular}

ASEBA, Achenbach System of Empirically Based Assessment; CBCL, Child Behavior Check List; ASR, adult self-report; borderline range (BR) for syndrome scale, T-score 65-70; clinical range (CR) for syndrome scale, T-score $\geq 70$; BR for broadband scale, T-score 60-63; CR for broadband scale, T-score $\geq 64$.

(ESS) at baseline were not associated with changes in HRQoL at follow up.

This significant increase in HRQoL at follow-up is in contrast to the findings of the prospective study of Sarkanen et al., [36], who found no such change after two years' follow-up. However, they reported a reduction in Rimon's Brief Depression Scale that they speculated might reflect adaptation to disease symptoms. The same explanation could apply to the improvement in Psychosocial Health seen in subjects with below-average HRQoL in our study. Yet, this could also arise by the statistical phenomenon of regression to the mean, although we observed considerable individual variation in the effect in the group with an HRQoL score less than the group mean. Our results are in contrast to a large population-based study of 1821 healthy children from Norway, aged 8-16 years, which showed that the overall total HRQoL decreased after six months of follow-up [45].

The association between the use of sodium oxybate and increased HRQoL at follow up is consistent with both the clinical impression and patients' own assessments. It is in line with the study of van Schie et al., [46], which showed that patients treated with sodium oxybate often report less daytime sleepiness and better alertness. Our findings are also supported by the report by Rocca et al., of patients treated with sodium oxybate having significantly better School Functioning than drug-naive NT1 patients [8].

Sodium oxybate, the salt of the neurotransmitter $y$-hydroxybutyrate (GHB), is currently the only available drug that can improve all of the core symptoms of narcolepsy. It is thought mainly to activate GABA type B receptors $[13,47,48]$. Its beneficial effects occur gradually and are markedly time-dependent [13], possibly taking 100-200 days to produce its maximum level of effect [49]. Thus, it is possible that patients using this drug at baseline might have experienced a better response to it when measuring HRQoL at follow-up. Its effect in NT1 patients underlines the complexity of the pathogenesis of NT1 and helps explain the heterogeneity of the disorder.

As expected, we found an inverse correlation at baseline between the ASEBA and PedsQL scores. In agreement with our findings, Inocente et al., [30] showed that depression in narcolepsy was the most important contributing factor to impaired HRQoL. However, our results revealed no relationship between the ASEBA score at baseline and change in PedsQL after two years of follow-up. Contrary to what is seen in other disease populations, the psychiatric challenges in children and young adults with narcolepsy have been considered to be a result of intrinsic dysfunction due to the loss of hypocretin neurons rather than due to psychiatric comorbidity [8].

Table 5

Possible predictors of changes in health-related quality of life (HRQoL).

\begin{tabular}{lll}
\hline & $\beta(95 \% \mathrm{CI})$ & $\mathrm{p}$ \\
\hline Sodium oxybate at T2 & $13.05(1.58,24.5)$ & $\mathbf{0 . 0 2 7}^{*}$ \\
Total ASEBA score & $0.52(-0.19,1.22)$ & 0.147 \\
Internalizing ASEBA score & $0.35(-0.35,1.10)$ & 0.309 \\
Externalizing ASEBA score & $0.36(-0.32,1.05)$ & 0.284 \\
ESS & $-0.18(-1.64,1.29)$ & 0.807
\end{tabular}

Linear regression, adjusted for age and gender. ESS, Epworth Sleepiness Scale; ASEBA, Achenbach System of Empirically Based Assessment.
We found no association between EDS at baseline and changes in HRQoL after two years of follow-up. Yet, it is possible that the tools used for measuring EDS in this and other studies are not sensitive enough to reflect the severity of subjective sleepiness in patients with narcolepsy. Some cross-sectional studies indicate an association of EDS with impaired HRQoL [8,35], while others have not found this association $[28,29,33]$.

Some factors that might influence our results need to be considered. First, although drawn from a bigger cohort, the sample considered in this study was small, with the consequent risk of selection bias and limited statistical power. Our findings are strengthened by the fact that all patients received the study invitation consecutively, and included patients received the same intervention consisting of confirmation of diagnosis, education about the disease, psychosocial evaluation, and medical and nonmedical treatment advice. We acknowledge that our findings would have been even more compelling if a control group had been included in the analysis. However, we could compare our results to national normative data, which further strengthened our results. Furthermore, discrepancies in clinical assessment of psychiatric comorbidity measured by ASEBA between multiple informants have been described [50-54]. In non-clinical study samples, youths tend to report a higher severity of symptoms than their parents [50,55]. Although, in a clinically-referred study population, parents are more likely to report a higher severity of symptoms [50]. A meta-analysis showed greater agreement between the ratings for younger than for older children [56]. We acknowledge that the use of both self-report and parent-report, depending on the age, could potentially be a limitation. However, due to the age span in our cohort, the use of either a self-reported version or a parentreported version only would have produced bias as well.

Narcolepsy is a heterogeneous disease with frequently reported psychiatric comorbidities that make treatment and follow-up a challenging task. Using the HRQoL survey and psychosocial evaluation as tools to enable better treatment and follow-up could be of great help to clinicians working in this field. Consequently, further studies exploring the impact of narcolepsy on quality of life and its interaction with psychiatric comorbidities are warranted.

\section{Acknowledgments}

Special thanks to Kåre Gautvik and Mona S. Heier for their engagement and critical reading of the article. Thanks to Janita Vevelstad and Rannveig Viste for their work with performance and interpretation of polysomnographies and multiple sleep latency tests. We would also like to thank David Swanson at Oslo Centre for Biostatistics and Epidemiology (OCBE) for statistical support. Last author Stine Knudsen is partially funded and first author Sebjørg Hesla Nordstrand is fully funded by research support from the Norwegian Ministry of Health and Care Services.

\section{Conflicts of interest}

This study was not an industry-supported. The authors have indicated no financial conflict of interest. 
The ICMJE Uniform Disclosure Form for Potential Conflicts of Interest associated with this article can be viewed by clicking on the following link: https://doi.org/10.1016/j.sleep.2018.05.037.

\section{References}

[1] American Academy of Sleep Medicine. (AASM) internation classification of sleep disorders. 3rd ed. Darien, IL: American Academy of Sleep Medicine; 2014.

[2] Yoss RE, Daly DD. Narcolepsy. Arch Intern Med 1960;106:168-71.

[3] Dauvilliers Y, Arnulf I, Mignot E. Narcolepsy with cataplexy. Lancet 2007:369(9560):499-511.

[4] Heier MS, Evsiukova T, Wilson J, et al. Prevalence of narcolepsy with cataplexy in Norway. Acta Neurol Scand 2009;120(4):276-80.

[5] Plazzi G, Pizza F. Childhood narcolepsy with cataplexy: a newly reported phenotype of an old disease? Sleep Med 2013;14(9):810-1.

[6] Poli F, Pizza F, Mignot E, et al. High prevalence of precocious puberty and obesity in childhood narcolepsy with cataplexy. Sleep 2013;36(2):175-81.

[7] Lecendreux M, Lavault S, Lopez R, et al. Attention-deficit/hyperactivity dis order (ADHD) symptoms in pediatric narcolepsy: a cross-sectional study. Sleep 2015;38(8):1285-95.

[8] Rocca FL, Finotti E, Pizza F, et al. Psychosocial profile and quality of life in children with type 1 narcolepsy: a case-control study. Sleep 2016;39(7): 1389-98.

[9] Ohayon MM. Narcolepsy is complicated by high medical and psychiatric comorbidities: a comparison with the general population. Sleep Med 2013;14(6):488-92.

[10] Jennum P, Ibsen R, Knudsen S, et al. Comorbidity and mortality of narcolepsy: a controlled retro- and prospective national study. Sleep 2013;36(6):835-40.

[11] Dauvilliers Y, Paquereau J, Bastuji H, et al. Psychological health in central hypersomnias: the French Harmony study. J Neurol Neurosurg Psychiatry 2009;80(6):636-41.

[12] Dodel R, Peter H, Spottke A, et al. Health-related quality of life in patients with narcolepsy. Sleep Med 2007;8(7-8):733-41.

[13] Kornum BR, Knudsen S, Ollila HM, et al. Narcolepsy. Nat Rev Dis Primers 2017:3:16100.

[14] Chen Q de Lecea L, Hu Z, et al. The hypocretin/orexin system: an increasingly important role in neuropsychiatry. Med Res Rev 2015;35(1):152-97.

[15] Peyron C, Tighe DK, van den Pol AN, et al. Neurons containing hypocretin (orexin) project to multiple neuronal systems. J Neurosci 1998;18(23): 9996-10015.

[16] Ruoff CM, Reaven NL, Funk SE, et al. High rates of psychiatric comorbidity in narcolepsy: findings from the burden of narcolepsy disease (BOND) study of 9,312 patients in the United States. J Clin Psychiatry 2017;78(2):171-6.

[17] Fortuyn HA, Mulders PC, Renier WO, et al. Narcolepsy and psychiatry: an evolving association of increasing interest. Sleep Med 2011;12(7):714-9.

[18] Fortuyn HA, Lappenschaar MA, Furer JW, et al. Anxiety and mood disorders in narcolepsy: a case-control study. Gen Hosp Psychiatry 2010;32(1):49-56.

[19] Modestino EJ, Winchester J. A retrospective survey of childhood ADHD symptomatology among adult narcoleptics. J Atten Disord 2013;17(7): 574-82.

[20] Inocente CO, Gustin MP, Lavault S, et al. Depressive feelings in children with narcolepsy. Sleep Med 2014;15(3):309-14.

[21] Heier MS, Gautvik KM, Wannag E, et al. Incidence of narcolepsy in Norwegian children and adolescents after vaccination against H1N1 influenza A. Sleep Med 2013;14(9):867-71.

[22] Partinen M, Saarenpaa-Heikkila O, Ilveskoski I, et al. Increased incidence and clinical picture of childhood narcolepsy following the 2009 H1N1 pandemic vaccination campaign in Finland. PLoS One 2012;7(3):e33723.

[23] Miller E, Andrews N, Stellitano L, et al. Risk of narcolepsy in children and young people receiving AS03 adjuvanted pandemic A/H1N1 2009 influenza vaccine: retrospective analysis. BMJ 2013;346:f794.

[24] Dauvilliers Y, Arnulf I, Lecendreux M, et al. Increased risk of narcolepsy in children and adults after pandemic H1N1 vaccination in France. Brain 2013;136(Pt 8):2486-96.

[25] Wijnans L, Lecomte C, de Vries C, et al. The incidence of narcolepsy in Europe: before, during, and after the influenza $\mathrm{A}(\mathrm{H} 1 \mathrm{N1})$ pdm09 pandemic and vaccination campaigns. Vaccine 2013;31(8):1246-54.

[26] Szakacs A, Hallbook T, Tideman P, et al. Psychiatric comorbidity and cognitive profile in children with narcolepsy with or without association to the H1N1 influenza vaccination. Sleep 2015;38(4):615-21.

[27] Pizza F, Peltola H, Sarkanen T, et al. Childhood narcolepsy with cataplexy: comparison between post-H1N1 vaccination and sporadic cases. Sleep Med 2014;15(2):262-5.

[28] Ozaki A, Inoue Y, Hayashida K, et al. Quality of life in patients with narcolepsy with cataplexy, narcolepsy without cataplexy, and idiopathic hypersomnia without long sleep time: comparison between patients on psychostimulants, drug-naive patients and the general Japanese population. Sleep Med 2012;13(2):200-6.

[29] Ozaki A, Inoue Y, Nakajima T, et al. Health-related quality of life among drugnaive patients with narcolepsy with cataplexy, narcolepsy without cataplexy, and idiopathic hypersomnia without long sleep time. J Clin Sleep Med 2008;4(6):572-8.

[30] Inocente CO, Gustin MP, Lavault S, et al. Quality of life in children with narcolepsy. CNS Neurosci Ther 2014;20(8):763-71.

[31] Daniels E, King MA, Smith IE, et al. Health-related quality of life in narcolepsy. J Sleep Res 2001;10(1):75-81.

[32] Flores NM, Villa KF, Black J, et al. The humanistic and economic burden of narcolepsy. J Clin Sleep Med 2016;12(3):401-7.

[33] Stores G, Montgomery P, Wiggs L. The psychosocial problems of children with narcolepsy and those with excessive daytime sleepiness of uncertain origin. Pediatrics 2006;118(4):e1116-23.

[34] David A, Constantino F, dos Santos JM, et al. Health-related quality of life in Portuguese patients with narcolepsy. Sleep Med 2012;13(3):273-7.

[35] Vignatelli L, Plazzi G, Peschechera F, et al. A 5-year prospective cohort study on health-related quality of life in patients with narcolepsy. Sleep Med 2011;12(1):19-23.

[36] Sarkanen T, Alakuijala A, Partinen M. Clinical course of H1N1-vaccine-related narcolepsy. Sleep Med 2016;19:17-22.

[37] Knudsen S, Jennum PJ, Alving J, et al. Validation of the ICSD-2 criteria for CSF hypocretin-1 measurements in the diagnosis of narcolepsy in the Danish population. Sleep 2010;33(2):169-76.

[38] Anic-Labat S, Guilleminault C, Kraemer HC, et al. Validation of a cataplexy questionnaire in 983 sleep-disorders patients. Sleep 1999;22(1):77-87.

[39] Rescorla LA. Assessment of young children using the Achenbach system of empirically based assessment (ASEBA). Ment Retard Dev Disabil Res Rev 2005;11(3):226-37.

[40] Reinfjell T, Diseth TH, Veenstra M, et al. Measuring health-related quality of life in young adolescents: reliability and validity in the Norwegian version of the Pediatric Quality of Life Inventory 4.0 (PedsQL) generic core scales. Health Qual Life Outcomes 2006;4:61.

[41] Varni JW, Burwinkle TM, Seid M. The PedsQL as a pediatric patientreported outcome: reliability and validity of the PedsQL Measurement Model in 25,000 children. Expert Rev Pharmacoecon Outcomes Res 2005;5(6):705-19.

[42] Novik TS. Validity of the child behaviour checklist in a Norwegian sample. Eur Child Adolesc Psychiatry 1999;8(4):247-54.

[43] Novik TS. Child Behavior Checklist item scores in Norwegian children. Eur Child Adolesc Psychiatry 2000;9(1):54-60.

[44] Diseth TH, Tangeraas T, Reinfjell T, et al. Kidney transplantation in childhood: mental health and quality of life of children and caregivers. Pediatr Nephro 2011:26(10):1881-92.

[45] Jozefiak T, Larsson B, Wichstrom L. Changes in quality of life among Norwegian school children: a six-month follow-up study. Health Qual Life Outcomes 2009;7:7.

[46] van Schie MK, Werth E, Lammers GJ, et al. Improved vigilance after sodium oxybate treatment in narcolepsy: a comparison between in-field and inlaboratory measurements. J Sleep Res 2016;25(4):486-96.

[47] Maitre M. The gamma-hydroxybutyrate signalling system in brain: organization and functional implications. Prog Neurobiol 1997;51(3):337-61.

[48] Huang YS, Guilleminault C. Narcolepsy: action of two gamma-aminobutyric acid type B agonists, baclofen and sodium oxybate. Pediatr Neurol 2009;41(1):9-16.

[49] Bogan RK, Roth T, Schwartz J, et al. Time to response with sodium oxybate for the treatment of excessive daytime sleepiness and cataplexy in patients with narcolepsy. J Clin Sleep Med 2015;11(4):427-32.

[50] Salbach-Andrae H, Klinkowski N, Lenz K, et al. Agreement between youthreported and parent-reported psychopathology in a referred sample. Eur Child Adolesc Psychiatry 2009;18(3):136-43.

[51] Achenbach TM. Manual for the youth self report form and 1991 profile. Burlington: University of Vermont, Departement of Psychiatry; 1991.

[52] Ferdinand RF, van der Ende J, Verhulst FC. Prognostic value of parentadolescent disagreement in a referred sample. Eur Child Adolesc Psychiatry 2006;15(3):156-62.

[53] Rescorla LA, Ginzburg S, Achenbach TM, et al. Cross-informant agreement between parent-reported and adolescent self-reported problems in 25 societies. J Clin Child Adolesc Psychol 2013;42(2):262-73.

[54] Salbach-Andrae H, Lenz K, Lehmkuhl U. Patterns of agreement among parent, teacher and youth ratings in a referred sample. Eur Psychiatry 2009;24(5): 345-51.

[55] Achenbach TM. Manual for the child behavior checklist/4-18 and 1991 profile. Burlington: University of Vermont, Departement of Psychiatry; 1991.

[56] Achenbach TM, McConaughy SH, Howell CT. Child/adolescent behavioral and emotional problems: implications of cross-informant correlations for situational specificity. Psychol Bull 1987;101(2):213-32. 



\section{ERRATA}

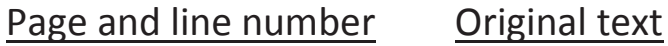

Page 9, line 23

Page 13 , line 5

Page 14, line 6

Page 17, line 5

Page 22, line21

Page 33, line 5

Page 39, line 16

Page 39, line 17

Page 55, line 23

Page 69, line 8

Page 79, reference 143 like thank

ton

in in

neurotrasmitter

interfer

associated obesity

patients that

questionnaire

Leceudreux

improved

Troost JJS...1993...

\section{Corrected text}

like to thank

tone

in

neurotransmitter

interfere

associated with obesity

patients who

questionnaires

Lecendreux

improvement

Troost J...Sleep. 1993... 